\title{
Cardiology 2008: 11th Annual Update on Pediatric Cardiovascular Disease - Abstracts
}

\section{Winner: 5th Annual Outstanding Investigator Award}

\section{Stress response in infants undergoing cardiac surgery with cardiopulmonary bypass and circulatory arrest \\ Robert Gajarski, Christopher Stefanelli, Joseph Graziano, Niko Kaciroti, John Charpie, Delia Vazquez}

University of Michigan, C.S. Mott Children's Hospital, Ann Arbor, MI, USA

Background: Glucocorticoids are frequently used for blood pressure support in infants with low cardiac output syndrome after heart surgery. However, the contribution of relative adrenocortical insufficiency to post-cardiotomy hemodynamic instability in infants with congenital heart disease has not been adequately studied. Methods: A prospective, observational cohort study was designed to test the hypothesis that postoperative inotropic support would parallel adrenocortical insufficiency in infants following heart surgery. Infants $<90$ days of age weighing $>2000$ gms who were undergoing cardiac operations were enrolled in the study and divided into 3 subgroups: cardiopulmonary bypass (CPB), cardiopulmonary bypass with deep hypothermic circulatory arrest (DHCA), and control subjects. All study patients (pts) underwent pre-operative cortrosyn $(\mathrm{ACTH})$ stimulation testing to verify adrenal competence. Serum cortisol was measured at 0, 2, 4, $8,12,18,24,36$ and 48 hours postoperatively. Serum ACTH and aldosterone (ALDO) levels were collected at less frequent time intervals. Relevant clinical data included: demographics (age, gender, and weight), cardiac diagnosis, surgical procedure, $\mathrm{CPB}$ and DHCA times, oxygen saturation and inotrope score (IS = Dopamine (dose in $\mathrm{mcg} / \mathrm{kg}$ / $\mathrm{min})+$ Dobutamine $(\mathrm{mcg} / \mathrm{kg} / \mathrm{min})+100 \quad[(\mathrm{epi}-$ nephrine $\mathrm{mcg} / \mathrm{kg} / \mathrm{min}$ ) + (norepinephrine $\mathrm{mcg} / \mathrm{kg} /$ $\mathrm{min}$ ) +15 (Milrinone $\mathrm{mcg} / \mathrm{kg} / \mathrm{min}$ ) +10000 (vasopressin $\mathrm{u} / \mathrm{kg} / \mathrm{min})$. Results: Fifty-eight study pts were enrolled and included $31 \mathrm{CPB}, 22 \mathrm{DHCA}$, and 5 controls. Ten DHCA pts received intra-operative steroids and were analyzed separately. All pts demonstrated adrenal competence preoperatively.
Cortisol levels peaked within 2 hours of surgery and, except for the DHCA-steroid group, there was no other group differences in peak levels. ACTH levels inversely correlated with bypass time in DHCA pts $(\mathrm{p}=0.03)$ but showed no correlation with circulatory arrest time. Inotrope scores were higher in pts undergoing more complex procedures $(\mathrm{CPB}$ and DHCA groups) compared to controls $(\mathrm{p}<0.001)$, but did not correlate with cortisol levels. ALDO (but not cortisol) levels were higher in DHCA compared to DHCA pts receiving steroids $(\mathrm{p}=0.036)$, and inversely correlated with inotrope score $(\mathrm{p}=0.06)$. ALDO levels were inversely correlated with oxygen saturation in controls $(p=0.01)$. Conclusions: Infants with congenital heart disease and an intact hypothalamic-pituitary-adrenal (HPA) axis demonstrated a predictable stress response to cardiac surgery. However, peak serum cortisol levels were unrelated to $\mathrm{CPB}$ or DHCA times, and did not predict the level of postoperative support (i.e. inotrope score). In contrast, ACTH and ALDO levels were inversely related to CPB time and inotrope score, respectively. Administration of intraoperative steroids had no significant impact on global cortisol response. In conclusion, the HPA axis appears to be intact in the majority of infants undergoing heart surgery, and the stress response is independent of CPB and DHCA.

Correspondence to Robert Gajarski: rjgaj@med.umich.edu

\section{Winner: 2nd Annual Nursing Scientist Award}

Enteral feeding algorithm for infants with hypoplastic left heart syndrome post Stage I Palliation

Nancy Braudis, Martha A. Q. Curley, Karen Beaupre, Kristi Thomas, Gina Hardiman, Peter Laussen, Ravi R. Thiagarajan

Children's Hospital, Boston, Massachusetts, USA

Background: Infants with hypoplastic left heart syndrome (HLHS) experience a high incidence of growth failure in the post-operative period following Stage I palliation. Because of an increased risk of necrotizing enterocolitis (NEC) in this population, 
clinicians may be reluctant to initiate early enteral feedings. Published guidelines for initiating and advancing enteral feedings in this population are limited. The purpose of this study was to develop and test the safety and efficacy of an enteral feeding algorithm in infants with HLHS post Stage I palliation. Methods: A multidisciplinary team including nurses, physicians and a nutritionist reviewed best practices and developed an enteral feeding algorithm. The algorithm describes a 4-step process identifying when to initiate and advance enteral feedings based upon residual gastric volume, abdominal girth and gastrointestinal distress. Once initiated, the goal of the algorithm is to facilitate the transition from full maintenance IV fluid to full maintenance enteral feedings $(100 \mathrm{cc} / \mathrm{kg} / \mathrm{day})$ within 16 to 20 hours. The algorithm was implemented on a prospective cohort of consecutive patients $>35$ weeks gestational age admitted over an 18 month period to the Cardiovascular ICU following stage I palliation of HLHS (study group). Excluded were nonsurvivors, patients supported on ECMO or those with a history of necrotizing enterocolitis and/or a fetal intervention. A retrospective chart review of consecutive patients admitted to the CICU over an 18 month period prior to the implementation of the feeding protocol was performed for comparison data (control group). Results: Sixty three infants with HLHS (study group $\mathrm{n}=36$, control group $\mathrm{n}=27$ ) were enrolled in the study. Age, gender, gestational age, Apgar scores, pediatric risk of mortality scores (PRISM-III) and type of congenital heart defect did not vary between the two groups. All patients in the control group received a Stage I with a BlalockTaussig (BT) shunt while only 33\% of the study group underwent a Stage I with a BT shunt $(\mathrm{p}<0.001)$. The remaining patients $(67 \%)$ in the study group underwent a Stage I with an RV-PA conduit. Duration of mechanical ventilation, length of ICU and hospital stay and nosocomial infection rates were similar for the two groups. The median duration of total parenteral nutrition (TPN) was significantly higher in the control (116 vs. 67.5 hours; $p=0.02$ ) compared to the study group. The median time to achieve recommended daily allowance of calories (RDA defined as $108 \mathrm{kcal} / \mathrm{kg} /$ day) was significantly reduced in the study group ( 7.5 vs. 10.5 days; $\mathrm{p}=0.02)$. Despite the rapid advancement of enteral feedings on the algorithm, there was no incidence of NEC in the study group ( $11 \%$ in the control group). Conclusions: The development and evaluation of this feeding algorithm indicates it is a safe and effective means by which enteral feeding can be successfully initiated and advanced in infants following complex congenital heart surgery.

Correspondence to Nancy Braudis: Dbpnancy@comcast.net

\section{Home surveillance program detects at-risk infants following Norwood palliation}

Nancy Rudd, Michelle Steltzer, Candace Buckles, Katherine Grochowski, Laurie Newton, Nancy Ghanayem, James Tweddell, Michele Frommelt, Peter Frommelt

\section{Medical College of Wisconsin; Children's Hospital of Wisconsin, Milwaukee, Wisconsin, USA}

Background: Infants discharged home following stage 1 palliation (S1P) for hypoplastic left heart syndrome (HLHS) possess at-risk hemodynamics. Due to well-described interstage vulnerability and death, we began a home surveillance program (HSP) in 2000 aimed at early detection of physiological variances believing subtle clinical changes could serve as warning signs of impending hemodynamic crises. Methods: Following hospital discharge parents perform a daily $\mathrm{O}_{2}$ saturation check and weight check. Parents are instructed to notify the cardiology team for any breach of pre-established criteria which include: $\mathrm{O}_{2}$ saturation $<75 \%$, failure to gain $\geqslant 10$ grams over 3 day span or weight loss of $\geqslant 30$ grams over 2 days. For tracking purposes, interstage events were classified as desaturation (desats) or inappropriate weight change (wt). Results: From October 2000 through February 2007, 103 HLHS pts were eligible for our HSP. There has been 100\% participation with no pts lost to follow-up. Seventyfour pts had S1P consisting of a modified BT shunt (Norwood) and 29 pts underwent RV $\rightarrow$ PA conduit (Sano). Length of home monitoring ranged from 15-165 days. 57\% (59/103) pts sought medical attention following breach of HSP criteria. 100 of $103(97 \%)$ of HSP pts survived to stage 2 palliation (S2P). Of the 3 deaths, all were sudden and without events prior to demise. Interstage events were detected in 44/74 (59\%) of Norwoods and 52\% $(15 / 29)$ Sanos. 94 total events were detected; 66 (70\%) were desats and 28 (30\%) were wt. 53/59 patients required admission because of HSP breach: some pts had multiple admissions (84 total). Of the 84 admissions, 78 (93\%) required additional medical or surgical intervention based on assessment at time of hospitalization. Conclusions: HSP is an effective monitoring system for identifying at-risk changes in physiology in infants discharged home following Norwood. Based on current HSP criteria for notification of medical personnel, $>90 \%$ of detected events resulted in significant medical and/or surgical intervention. HSP should be standard practice in the care of this high risk population.

Correspondence to Nancy Rudd: nrudd@chw.org 
Where's the evidence? Paucity of high level evidence for therapy in pediatric cardiology

Selvi Senthilnathan, Gautham Suresh

Dartmouth-Hitchcock Medical Center, Lebanon, NH, USA

Background: In the hierarchy of evidence supporting therapeutic interventions, randomized controlled trials (RCT's) comprise the highest level of evidence, with studies using non-randomized controls, and those with case control, cohort, case series and case report study designs comprising progressively lower levels of evidence. The extent to which therapies used in pediatric cardiology, including medications, percutaneous interventions and surgery, are supported by high-level evidence is unknown. Therefore we aimed to determine the levels of evidence available in the literature for therapeutic interventions in pediatric cardiology. Methods: We manually reviewed either the abstract or the full text of all consecutive publications (identified through a Pubmed search) over a threeyear period (Aug 2004-Aug 2007) on therapeutic interventions for cardiac disease in children. We analyzed only articles with an English abstract or English full text available, and excluded articles that were unrelated to therapy, described adult patients predominantly, or described basic science research. We classified eligible articles according to year and country of publication, type of cardiac disease, type of therapeutic intervention, and study design. We also searched for all randomized trials in the past ten years in pediatric cardiology, neonatology and on ischemic heart disease in adults. Results: Of 4332 articles published in three years, 3317 qualified for inclusion. The countries of publication were: USA 31\%, Japan 9\%, Germany $7 \%$, UK $6 \%$, Italy $4 \%$, other countries $41 \%$, and multi-country $2 \%$. The diseases primarily addressed by these articles were: congenital heart disease (CHD) 60\%, acquired heart disease (AHD) 12\%, heart failure $12 \%$, arrhythmias $11 \%$, and multiple diseases $5 \%$. Therapeutic interventions addressed by these studies were: surgery $49 \%$, percutaneous interventions (PCI) 23\%, medications 9\%, circulatory support $5 \%$, other forms of therapy $4 \%$, and multiple forms of therapy $11 \%$. Among primary studies, those providing low (case reports and case series), medium (case control, cohort, and nonrandomized controlled studies) and high (RCT's) levels of evidence comprised 52\%, $45 \%$ and $3 \%$ respectively. RCT's comprised between 2-3\% of studies in all quartiles of this dataset of sequential studies, indicating no significant shift over time.
RCT's comprised $2 \%$ of all studies on heart failure and CHD, and $3 \%$ of studies on AHD and arrhythmias. RCT's comprised $11 \%$ of studies on medications, but only $2 \%$ of studies on PCI and, and just $1 \%$ of studies on surgery. Of the RCT's, $28 \%$ were published from the US. The commonest disease categories addressed by these RCT's were CHD (57\%), AHD (14\%), and arrhythmias (12\%). The commonest therapeutic categories addressed by these RCT's were medications (38\%), surgery (20\%), and PCI $(16 \%)$. Over a ten year period, there were 213 RCT's in pediatric cardiology, 3263 in neonatology, and 5394 on adult ischemic heart disease. Conclusions: There is a paucity of high level evidence to support therapeutic interventions in pediatric cardiology particularly for percutaneous interventions and surgery. There is a great need for clinical research in pediatric cardiology that will produce high level evidence for common conditions and commonly used therapies. The creation of collaborative research networks can foster such research.

Correspondence to Selvi Senthilnathan: selvi.senthilnathan@ gmail.com

\section{A Modification to the Ross procedure that may eliminate late autograft dilatation}

Joylyn Headings, ${ }^{1}$ Ross M. Ungerleider, ${ }^{1}$ Karl F. Welke, ${ }_{1}$ Irving Shen, ${ }^{2}$ Matt Slater, Howard Song ${ }^{1}$

${ }^{1}$ Oregon Health \& Science University, Portland, OR, USA, ${ }^{2}$ Fairfax Hospital, Fairfax, VA, USA

Background: According to the STS database, the use of pulmonary autograft replacement of the aortic valve (Ross Procedure $\square$ RP) in adult patients has declined over the past several years. A contributing factor for this decline may be concern regarding autograft dilatation that has been described in $15-20 \%$ of patients. It has been demonstrated in large series that in affected patients this dilatation can progress relentlessly at $0.5-1.0 \mathrm{~cm}$ per year and can be accompanied by significant autograft insufficiency leading to the need for reoperation. Methods: Beginning in October 2004, we began performing RP in adult $(>18 \mathrm{y} / \mathrm{o})$ patients $(\mathrm{n}=22)$ using a modified technique (MRP) in which the autograft was completely encased in a Dacron graft $(28-32 \mathrm{~mm})$ prior to implantation. The technical features of the procedure have become reproducible and reliable and have evolved over time. Autografts were evaluated for function and for the incidence of root dilatation (aortic root diameter $>4.0 \mathrm{~cm}$ ) with 
serial echocardiograms and compared to a cohort of similar adult patients who received a standard RP between 1/01-10/04 $(\mathrm{n}=27)$. Results: There was no operative mortality. Patients in both groups were similar with respect to age, type of aortic disease (A.S.; A.I. or both) and size of initial autograft. Follow-up data were available for $21 / 27$ patients in the RP group and 18/22 patients in the MRP group. Two Patients in the RP group required repeat surgery for severe A.I. and both received prosthetic valves. There was one patient in the MRP group who developed severe A.I. and returned to us shortly after surgery. This was related to a technical error and the autograft was successfully repaired. Autograft dilatation (aortic root at sinotubular junction $>4.0 \mathrm{~cm}$ ) was found in 8 of the remaining 19 patients (42\%) with RP and in none $(0 \%)$ of the 18 patients with MRP. Average autograft diameter in the RP group was $40.3+7.6 \mathrm{~mm}$; range $25-$ $56 \mathrm{~mm}$. Average autograft diameter in the MRP group was $32.2 \mathrm{~mm}+3.5 \mathrm{~mm}$; range $26.6-37 \mathrm{~mm}$. Conclusions: MRP is reproducible using techniques that have evolved over the past 3 years. It results in excellent autograft function and appears to restrict autograft dilatation. Clearly, longer follow-up will be required to ensure that these findings hold up over time. However, these results are extremely encouraging and may lead to restored enthusiasm for the RP in adult patients who require aortic valve replacement.

Correspondence to Joylyn Headings: headings@ohsu.edu

\section{Pregnancy outcome in women with tetralogy of Fallot}

Lia Pedersen, Thais Pedersen, Hanne Ravn, Vibeke Hjortdal

\section{Aarbus University Hospital, Aarbus, Denmark.}

Background: Surgical results after repair of tetralogy of Fallot have remained excellent for the last decades with current long-term survival rates over 95\%. Since functional capacity, quality of life and social interactions are basically normal in this large group of patients, pregnancy obviously becomes a relevant issue for the female subgroup. In consequence, adequate obstetrical and cardiological management of pregnancy is particularly important. The objective of this study is to describe pregnancy outcome and fertility in a single center series of women who underwent surgery for tetralogy of Fallot. Methods: Data on 78 women operated for tetralogy of Fallot between 1972-92 at Aarhus University Hospital, Denmark, were obtained from hospital records and national registries. In addition the current survivors were asked to answer a questionnaire. Results: Follow-up data was obtained for $100 \%$ of the women. Of 58 women who reached an age of at least 18 years ( 45 current survivors, 13 who succumbed in adult age), 54 pregnancies in 25 women were registered. Spontaneous abortion rate was $15 \%$ and infertility rate was $3.4 \%$. There has been 41 life births, with a median birth weight $3.2 \mathrm{~kg}$. Only one newborn was small for gestational age $(2 \%)$ and no one was born before 36th week. The recurrence rate of congenital heart disease was high $(9.8 \%)$. Cardiac complications during of after pregnancy were not observed and only one woman had pre-eclampsia. Conclusions: Pregnancy is well tolerated in women with tetralogy of Fallot and an excellent neonatal outcome is expected. The recurrence risk of congenital heart disease, most often tetralogy of Fallot, is high.

Correspondence to Lia Pedersen: lia-kris@dadlnet.dk

Interstage echocardiographic changes in patients undergoing Hybrid Stage I palliation for hypoplastic left heart syndrome

Bernadette Fenstermaker, Glen E. Berger, Daniel G. Rowland, John Hayes, Sharon L. Hill, John P. Cheatham, Mark Galantowicz, Clifford L. Cua

The Heart Center, Nationwide Children's Hospital, Division of Pediatrics and Cardiothoracic Surgery, Columbus, Obio, USA

Background: The hybrid procedure consisting of bilateral pulmonary artery bands, ductus arteriosus stent, and atrial septostomy is an alternative for initial palliation of patients with hypoplastic left heart syndrome (HLHS). No echocardiographic data exists for the inter-stage period. The goal of this study was to describe echocardiographic changes during this period. Methods: Chart review was performed on patients discharged from the hospital with the diagnosis of HLHS who underwent hybrid Stage I palliation. Patients were excluded if they were followed at another institution or if they had not undergone the second stage procedure. Echocardiograms at hospital discharge, pre and post any interstage intervention, and prior to Comprehensive Stage II procedure were reviewed. Bilateral pulmonary artery (RPA and LPA) velocity, slope, velocity time integral (VTI), pressure halftime $(\mathrm{p} 1 / 2)$, and systolic/diastolic (S/D) ratio of the 
waveforms were recorded. ASD mean gradient, PDA velocity, retro-aortic arch velocity, tricuspid regurgitation (TR), and right ventricular function were documented. Exploratory hypotheses were tested with Chi Square and t-tests. Stepwise logistic regression was used to identify any multiple sets of relatively independent variables. Results: Between $7 / 02$ to $12 / 06,30$ patients met inclusion criteria. Fourteen patients underwent 22 different interventions at the atrial septum, PDA, or retro-aortic arch in the interstage period. Baseline ASD gradient $(p=0.012)$ and PDA velocity $(p=0.002)$ predicted an interstage intervention. There were significant differences in LPA and RPA VTI $(\mathrm{p}=0.011,0.03), \mathrm{p} 1 / 2 \quad(\mathrm{p}=0.038,0.008)$, and S/D ( $=0.012,0.033) ;$ RPA slope $(\mathrm{p}=0.013)$; ASD gradient $(\mathrm{p}=0.003)$; PDA velocity $(0.021)$; and TR (0.031) pre and post an intervention. There were significant differences in baseline vs. comprehensive Stage II LPA and RPA VTI $(\mathrm{p}=<0.001,<0.001)$ and $\mathrm{p} 1 / 2(\mathrm{p}=0.001,0.001) ;$ and $\mathrm{S} / \mathrm{D}(\mathrm{p}=0.034$, $0.005)$; LPA velocity $(\mathrm{p}=<0.001)$; PDA velocity $(\mathrm{p}=<0.001)$; and retro-aortic velocity $(\mathrm{p}=<0.001)$. Pre-comprehensive stage II function tended to predict death $(p=0.085)$. Conclusions: Echocardiographic parameters help predict interstage course and guide clinical therapy for this patient population.

Correspondence to Clifford L. Cua: clcua@hotmail.com

\section{Non-contrast enhanced magnetic resonance angiography (NC-MRA) is a viable diagnostic alternative to conventional contrast enhanced MRA (CE-MRA) in children}

Keren Hasbani, Pamela S. Vincent, Carolyn A. Bondy, Steven Shea, Andrew E. Arai

National Institutes of Health, DHHS, Children's

National Medical Center, Siemens, USA

Background: In light of recent concerns about the safety of radiation associated with CT scans and MRI contrast agents, there is increasing need for non-contrast enhanced magnetic resonance angiography (NC-MRA), particularly for pediatric subjects. The aim of this study was to assess the quality and feasibility of NC-MRA. In 15 patients referred for thoracic angiography, we compared NCMRA with conventional contrast enhanced MRA (CE-MRA). To assess feasibility of NC-MRA in children, we performed NC-MRA in 49 consecutive pediatric subjects. Methods: Fifteen patients (17-73 years old) underwent both NC-MRA and CE-MRA for comparison purposes. Forty-nine children (8-18 years old) who were undergoing thoracic vascular assessment as part of research studies had a NC-MRA performed without sedation, without an intravenous catheter, and without contrast. NC-MRA used navigator-gating on the diaphragm to compensate for respiratory motion and ECG-gating to minimize cardiac motion artifacts. CE-MRA was performed in a breathhold but was not ECG-gated. Image quality was scored from $4=$ excellent, $3=\operatorname{good}, 2=$ fair, $1=$ poor, and $0=$ non-diagnostic for diagnosing anatomy and abnormalities in the following locations: aortic root, aortic arch, descending aorta, pulmonary veins, and the left ventricle. All the patients were scanned on a 1.5 Tesla MRI scanner (Siemens) and signed consent/assent for research studies approved by the local institutional review board. Results: In the 15 patients that underwent both NC-MRA and CE-MRA, image quality was equivalent for the aortic arch $(3.9 \pm 0.2$ vs $3.9 \pm 0.4$, $\mathrm{NS})$, the descending aorta $(3.9 \pm 0.3$ vs $3.9 \pm 0.3$, $\mathrm{NS})$, and the pulmonary veins $(3.4 \pm 0.6$ vs $3.3 \pm$ 0.6 , NS). However, image quality was superior for NC-MRA in the aortic root compared with CE-MRA $(3.7 \pm 0.4$ vs $1.7 \pm 0.6, \mathrm{p}<0.001)$ and also in the left ventricle $(3.6 \pm 0.4$ vs $1.7 \pm 0.7, \mathrm{p}<0.001)$. On the other hand, the CE-MRA was performed in typically $<15$ seconds while the NC-MRA took 8-15 minutes. In the 49 consecutive pediatric patients that had NC-MRA, 2 studies were non-diagnostic, 0 were poor in quality, 6 were fair quality, and the remaining 41 studies were of good or excellent quality. Conclusions: NC-MRA provides a high quality angiographic assessment of the thorax that is feasible in pediatric patients over the age of 8 and can eliminate the need for radiation, gadolinium, or anesthesia. In areas with significant cardiac motion, the NC-MRA is superior to conventional breathhold CE-MRA. The NC-MRA has significant disadvantages in terms of the length of the acquisition and the $4 \%$ failure rate.

Correspondence to Keren Hasbani: khasbani@yahoo.com

\section{Truncus arteriosus repair: mid-term results} using a modified technique

James Gangemi, Jill M. Cholette, George M. Alfieris

University of Rochester Medical Center, Rochester, NY, USA

Background: Surgical management of truncus arteriosus has evolved over the years, both in technique and timing of repair. Complete repair early in life, particularly during the neonatal period, has become 
standard of care in most high volume centers. We present our experience with early repair of truncus arteriosus using a modification of a technique first described by Drs. Barbero-Marcial and Jatene in 1990, which preserves pulmonary artery architecture. Methods: From August 1995 to June 2006, 16 patients underwent complete repair of truncus arteriosus using our modified technique. Median weight was $3.3 \mathrm{~kg}$ (range 2 to $4.6 \mathrm{~kg}$ ). Median age was 21 days (range 3 to 84 days). Nine patients $(56 \%)$ were operated on during the neonatal period ( $<30$ days). Associated anomalies included moderate truncal valve regurgitation in 3 patients (19\%), interrupted aortic arch in 3 patients (19\%), DiGeorge syndrome in 3 patients (19\%), and 1 patient (6\%) with prior omphalocele repair. All surgeries were performed using a modified technique which involved a "hockeystick" incision across the truncal root, closing the aorto-pulmonary window with a gortex patch, closing the ventricular septal defect through a right ventriculotomy, and placing an aortic $(\mathrm{n}=15)$ or pulmonary $(\mathrm{n}=1)$ homograft (median conduit diameter $12 \mathrm{~mm}$ ) as a right ventricular (RV) to pulmonary artery (PA) conduit. This technique does not require transection of the aorta and leaves the pulmonary arteries in their native position. Mean cardiopulmonary bypass and aortic crossclamp times were 191 minutes and 128 minutes, respectively. Five patients $(31 \%)$ required some form period of deep hypothermic circulatory arrest (DHCA). The initial 2 patients underwent DHCA prior to modification of our technique to include bicaval cannulation and normothermia. The remaining 3 patients required concomitant repair of interrupted aortic arch. No truncal valve repairs or replacements were necessary at the time of initial surgery. Results: There were no in-hospital deaths. There were no bleeding or other cardiac complications. No patients required extracorporeal membrane oxygenation. Median duration of mechanical ventilation was 3 days (range 1 to 9 days). Median ICU length of stay was 8 days (range 5 to 18 days). Survival in this series was 94\%; one patient died at home at 9 months of age of a presumed respiratory event. Median follow-up was 3.5 years (range 6 months to 8.5 years). Only one of the 15 surviving patients required an RV to PA conduit replacement at 5.5 years of age for progressive conduit stenosis. Conclusions: Early repair of truncus arteriosus using this modified technique can be performed successfully with low surgical morbidity and mortality as well as very acceptable mid-term re-operation rates. We believe this technique preserves PA architecture, allows for normal growth and prevents PA bifurcation and proximal branch stenosis.

Correspondence to James Gangemi: Jay_Gangemi@urmc.rochester.edu
Feeding methods and growth trajectories in infants following Norwood and NorwoodSano procedures

AnnMarie Reid, Britt Pados, Evelyn Paz

Morgan Stanley Children's Hospital of New York Presbyterian, NY, USA

Background: Infants with single ventricle cardiac physiology following the Norwood or the NorwoodSano procedure often experience growth failure in the first year of life. Growth failure at this time of critical brain growth has significant implications on neurodevelopmental outcome. Currently there are few evidence based feeding strategies that support appropriate growth for these infants. The purpose of this study was to determine whether a continuous or bolus feeding regimen was more effective for promoting growth in infants following the Norwood or Norwood-Sano procedure. Methods: A retrospective chart review was performed on patients who were in the neonatal intensive care unit and underwent the Norwood or Norwood-Sano procedure at a single institution between July 1, 2005 and June 30, 2007. A total of 34 infants were included in the study. Demographic data, measurements, surgical data, and feeding information were collected. The infants were then divided into two groups, continuous or bolus, based on feeding mode at the time that full enteral feeding volume (defined as $150 \mathrm{ml} / \mathrm{kg} /$ day) was obtained. The continuous feeding group included infants that received 12 hours or 24 hours of continuous feeds. A number of parameters were used to determine the efficacy of each feeding regimen. These parameters were: discharge weight; discharge length; discharge head circumference; time to first feed; time to full feeds; length of hospital stay; day of life that birth weight was regained; weight gain from full feeds to discharge; overall weight gain; and number of days from full feeds to discharge. Means between the two feeding groups and multiple two sample t-tests were performed. Significance was defined as $\mathrm{p}<0.05$. Results: There was no significant difference between the two groups with regard to many of the outcome variables. However, two variables did reach statistical significance, including discharge weight and overall weight gain per day. Mean discharge weight was significantly greater for the continuous feeding group than for the bolus feeding group (3622.5 grams versus 3040.8 grams, $\mathrm{p}=0.004$ ). However, the mean birth weights of both groups were also different (bolus group, 2870.75 grams; continuous group, 3221.429 grams, $\mathrm{p}=0.04)$. Despite the 
differences in birth weight, the continuous feeding group gained a larger amount of weight between birth and discharge than did the bolus feeding group (401.071 grams versus 170.05 grams). The overall weight gain per day, defined as: (discharge weightbirth weight)/(length of stay) reached statistical significance. Infants in the continuous feeding group achieved greater mean weight gain per day than did the bolus feeding group (11.536 grams vs. 5.803 grams, $\mathrm{p}=0.049)$. Conclusions: Infants with single ventricle congenital heart disease following Norwood and Norwood-Sano procedures achieve superior growth when fed a continuous feeding regimen, as opposed to exclusive bolus feedings. Further research in the form of a large, prospective, controlled trial is necessary to confirm these results.

Correspondence to AnnMarie Reid: and9035@nyp.org

\section{Routine mechanical ventricular assist following the Norwood procedure improved cerebral perfusion}

Jamie You, Brian Mejak, Carmen Giacomuzzi, Eileen Heller, Ross Ungerleider, Karl Welke

Division of Pediatric Surgery, Doernbecher Children's Hospital, Oregon Health E Science University, Portland, OR, USA

Background: Previous studies have demonstrated excellent survival following the Norwood procedure for palliation of hypoplastic left heart syndrome (HLHS) by the routine use of a ventricular assist device. However, assessment of adequate cerebral perfusion remains a challenge after surgery. The present study used near infrared spectroscopy (NIRS) to measure the cerebral oxygen saturation $\left(\mathrm{ScO}_{2}\right)$ peri-operatively. Other parameters including the somatic NIRS oxygen saturation $\left(\mathrm{SsO}_{2}\right)$, lactic acid, and mean arterial pressure (MAP) were also assessed during the period ventricular assist device (VAD) support. Methods: Twenty-nine consecutive infants, with an average age of $14.3 \pm 21.6$ days and an average weight of $3.3 \pm 0.6 \mathrm{~kg}$, undergoing Norwood operation for HLHS were routinely placed on a ventricular assist device (VAD) immediately following modified ultrafiltration. We used the pre-existing cannulas from cardiopulmonary bypass (CPB) that were in the right atrium and the neoaorta to support the patients on ventricular assist. VAD flows were maintained at approximately $146.9 \pm 31.1 \mathrm{~mL} / \mathrm{kg} / \mathrm{min}$. The duration of VAD averaged $50.5 \pm 40.7$ hours. $\mathrm{ScO}_{2}, \mathrm{SsO}_{2}, \mathrm{MAP}$ and lactic acid were continuously monitored from time of intubation to withdrawal of VAD support. Data from the post-induction period to 24-hour postoperatively were analyzed. Data points collected included ten minutes prior to $\mathrm{CPB}$, ten minutes prior to termination of CPB, MUF, time before VAD initiation, and hourly measurements on VAD. Results: Average MAPs for the study were 35.0 \pm $6.9 \mathrm{mmHg}$ (pre-CPB), $46.0 \pm 9.8 \mathrm{mmHg}$ (prior to VAD initiation), $64.4 \pm 18.5 \mathrm{mmHg}$ (two hours postVAD), and $51-54 \mathrm{mmHg}$ for the duration of the first twenty four hours on VAD support. Baseline $\mathrm{ScO}_{2}$ values were $60.5 \pm 12.4 \%$ and rose to an average of $72.0113 .1 \%$ on $\mathrm{CPB}$. $\mathrm{ScO}_{2}$ prior to VAD initiation was $47.0 \pm 13.0 \%$ and increased to $49.9 \pm 12.1 \%$ upon initiation. $\mathrm{ScO}_{2}$ values dropped to its lowest point $(45.6 \pm 13.3 \%)$ after two hours of ventricular assist but were significantly higher $(\mathrm{p}<0.01)$ at hour 12 of support $(45.6 \pm 13.3 \%$ vs. $55.1 \pm 9.3 \%) . \mathrm{SsO}_{2}$ values ranged from $65.7 \pm 19.2$ to $75.4 \pm 7.1 \%$ during the twenty four hour study period. Lactic acid levels peaked following modified ultrafiltration at $6.8 \pm$ $2.2 \mathrm{mmol} / \mathrm{L}$, but dropped significantly $(\mathrm{p}<0.01)$ to pre-operative levels $(1.9 \pm 0.8 \mathrm{mmol} / \mathrm{L})$ by hour 18 of VAD support $(2.1 \pm 0.7 \mathrm{mmol} / \mathrm{L})$. All patients were successfully weaned off VAD. The hospital survival rate was $82 \%$. Conclusions: $\mathrm{ScO}_{2}$ continually increased during the first twenty-four hours of routine postoperative VAD following the Norwood operation. In addition to increased $\mathrm{ScO}_{2}$, tissue perfusion was improved during VAD support reflected by decreasing lactate levels and stabilized $\mathrm{SsO}_{2}$ levels. It could simplify postoperative management, lead to excellent hospital survival, and augment cerebral oxygen delivery, resulting in improved neurologic outcomes for this challenging group of patients.

Correspondence to Jamie You: youj@ohsu.edu

Somatic growth and oxygenation following first-stage palliation for hypoplastic left heart syndrome

DeeAnn Martin, Robert Jaquiss, Michiaki Imamura, Stephanie Rockett, Trenda Ray

Arkansas Children's Hospital E University of Arkansas for Medical Sciences, Little Rock, AR, USA

Background: Despite remarkable improvements in hospital survival for patients undergoing first stage palliation (S1P) for hypoplastic left heart syndrome and related conditions, interstage mortality rates of $5-20 \%$ persist. Little information is available 
about growth rates and changes in oxygen saturation for children in the interstage period. We initiated a program for home monitoring of weights and oxygen saturation for S1P survivors in hopes of reducing interstage mortality and learning more about interstage growth and development. Methods: Survivors of BT shunt-type S1P (BTS1P), $\mathrm{n}=12$, and Sano-type S1P (RVS1P), $\mathrm{n}=32$, were discharged with a baby scale, pulse oximeter, and instruction to record daily weights and oxygen saturation. Parents and home health agencies were given pre-specified criteria to contact their physicians for, and routine inter-stage clinic appointments were followed. Home monitoring data were sent to the home surveillance office and cardiologist weekly. Unplanned interventions, deaths, daily parameter values, and the timing of second stage operation ( $22 \mathrm{P})$ were noted. The timing of S2P was at the discretion of the attending cardiologist. Results: The slopes of linearized growth curves (weight gain per day) did not differ between groups (BTS1P $17 \mathrm{gm} /$ day, RVS1P $19 \mathrm{gm} /$ day, $\mathrm{p}=\mathrm{NS}$ ). Several individuals demonstrated a weight gain plateau at approximately 90-120 days. Oxygen saturations declined in the interstage period in the RVS1P patients $(-0.05 \%$ per day) and increased in the BTS1P patients $(+0.02 \%$ per day), although the difference did not achieve statistical significance. Early declines in oxygen saturation led to shunt revision in two BTS1P patients, but not in any RVS1P patients. Two RVS1P patients with poor weight gain were admitted to the hospital and eventually underwent early S2P. There were 3 interstage deaths in the RVS1P group (at 68, 86, and 98 days) without preceding alteration in weight gain or oxygenation. One RVS1P patient with pulmonary hypoplasia was felt to not be a candidate for S2P and died at 408 days. There were no interstage deaths in the BTS1P group. During the study period the mean annual age at $\mathrm{S} 2 \mathrm{P}$ declined from 188 days to 128 days. Conclusions: Home monitoring may identify early shunt dysfunction in BT-type S1P patients. Interstage weight gain after either type of S1P is approximately 18 grams per day and seems to reach a plateau value. Interstage death was not eliminated by the initiation of a home monitoring program, although the observation of a weight gain plateau for individual patients leading to earlier S2P may reduce interstage attrition.

Correspondence to DeeAnn Martin: MartinDeeAnn@uams.edu

\footnotetext{
A new modification of the PTFE bicuspid pulmonary valve - early echocardiography evaluation
}

Cheri Meckley, Alfred Asante-Korang, J. Blaine John, Tina Morolla, Kathryn Carpentieri, Jeffrey Jacobs, Paul Chai, James Quintessenza

\section{All Children's Hospital and St. Joseph's Hospital, St Petersburg, FL, USA}

Background: Chronic pulmonary insufficiency results in RV enlargement and dysfunction and eventually RV failure and arrhythmias. Multiple surgical phases for pulmonary valve replacement have resulted in recurrence of RVOT obstruction and pulmonary insufficiency. Earlier experience with the $0.6 \mathrm{~mm}$ PTFE bicuspid valve showed considerable improvement with low incidence of reoperation, significant valve stenosis, or insufficiency. Our experience using $0.6 \mathrm{~mm}$ PTFE to construct a bicuspid valve resulted in a low incidence of reoperation, significant valve stenosis, or insufficiency. Some valve leaflets however, developed thickening and decreased mobility over time, presumably related to cellular in growth. We report our echocardiographic experience with the newly revised $0.1 \mathrm{~mm}$ PTFE bifoliate valve. Methods: Retrospective echo study to evaluate function of new gortex bicuspid pulmonary valve replacement $0.1 \mathrm{~mm}$ in tetralogy of fallot and truncus patients with pulmonary valvotomy. Mean pulmonary valve Doppler gradients will be obtained on 12 consecutive patients initial post op echo, 6 months, and 12 months follow up. The pulmonary insufficiency was graded on a scale from $0-4$ based on severity. Results: A total of 12 patients have undergone the PTFE $(0.1 \mathrm{~mm})$ valve procedure (Age 4-59 yr, median 16.4). All the patients had no or only trace PI following surgery. $66.6 \%$ of patients had no residual pulmonary stenosis. $25 \%$ of patients had mean gradient of 10 to $20 \mathrm{mmHg}$, and $8.3 \%$ had mean gradient of 20 to $30 \mathrm{mmHg}$. Conclusions: Short term results of the newly modified $0.1 \mathrm{~mm}$ PTFE pulmonary valve are excellent. There is a high success rate of near normal valve function with absence of PI and only minimal gradient across the PV by echocardiogram. Based on the follow-up data on the older forms of PTFE valves, it is anticipated that these patients would continue to have minimal recurrence of PI or stenosis.

Correspondence to Cheri Meckley: moncheri20@yahoo.com

\section{Is lipoprotein (a) a risk marker of coagulation abnormalities in patients with single ventricle physiology?}

Nina Hakacova, Martin Zahorec, Zuzana LaluhovaStriezencova

Department of Pediatric Cardiology, Children's Cardiac Center, Bratislava, Slovak Republic 
Background: Concentration of Lipoprotein (a) [Lp (a)] is genetically determined and plays an important role in childhood thrombosis. Patients with single ventricle (SV) physiology suffer from recurrent thrombotic events. The relevant risk markers of thrombosis in this population are still missing. The aim of the study was to test the hypothesis, that increased Lp (a) levels are associated with hemocoagulation abnormalities in SV patients. Methods: After obtaining institutional ethical committee approval and informed parental consent, 60 patients with SV physiology admitted to Children's Cardiac Center in Slovakia between October 2005 and September 2007 were included into the study. Fifty healthy children were assessed as age-matched controls. Groups did not differ significantly in demographic characteristics. Concentration of Lp (a) was assessed as prothrombotic risk marker using a cut-off value of $300 \mathrm{mg} / \mathrm{L}$. Concentration of Factor (F) II, FV, FVII, FVIII, Protein (P) C, PS and Antithrombin (AT) were measured before the surgical procedure and evaluated as possible predictors of hemocoagulation disturbances. Laboratory signs of hemocoagulation state disturbance were assessed by measuring concentration of Plasminogen, Fibrin Degradation Products (FDP) and D-dimer. Correlation of $\mathrm{Lp}$ (a) concentration with laboratory signs of hemocoagulation state disturbance was tested. Results: Concentration of Lp (a) reached levels under the cut-off value in children of both SV and Control group, except of one patient with SV physiology whose concentration of Lp (a) was $500 \mathrm{mg} / \mathrm{L}$. Coagulation Factor (F) II $(\mathrm{p}<$ $0.0001)$, FVII $(\mathrm{p}<0.001)$, F VIII $(\mathrm{p}<0.01)$ reached significantly lower values in SV group compared with Control group. Anticoagulation Protein (P) C $(p<0.001)$, PS $(p<0.01)$ and Antithrombin III $(\mathrm{p}<0.001)$ were significantly lower in SV patients. Both pro- and anticoagulation factors were under the low limit for age in the SV group. SV group had significantly higher levels of D-dimer $(\mathrm{p}<0.0001)$ and FDP $(\mathrm{p}<0.0001)$ and significantly lower levels of Plasminogen $(\mathrm{p}<0.0001)$ in comparison with Control group. Levels of Lp (a) did not correlate with laboratory signs of prothrombotic state. Conclusions: Normal concentration of Lp (a) in association with hemocoagulation factor disturbances and laboratory signs of thrombosis in SV patients suggest that $\mathrm{Lp}$ (a) is not relevant risk marker of prothrombotic state in this population. We conclude that importance of detailed monitoring of hemocoagulation factors before surgery is necessary in children with SV physiology to assess thrombotic risk.

Correspondence to Nina Hakacova: nina.hakacova@gmail.com

\section{Safe feeding of infants with hypoplastic left heart syndrome requires a proactive multidisciplinary approach}

Coleen Miller, Bronwyn Bartle, Kelly Nicklas, Karen Buckmiller, Pat Clark, Susan Dixon, Susan Fussell, Cathy Gustafson, Laura Oliver, Renee Williams, Maura Baldwin, James Jaggers, Jon Meliones, Piers Barker

Duke University Medical Center, Durbam, NC, USA

Background: Over the past decade mortality rates of infants with hypoplastic left heart syndrome (HLHS) undergoing staged palliation has significantly decreased due to improved surgical and critical care techniques especially after the first surgical stage. Despite this decreased mortality, HLHS remains associated with considerable morbidity. In particular, these infants are prone to gastrointestinal complications which clinically manifest as problems with poor oral coordination, gastroesophageal reflux, aspiration, or even the most serious, necrotizing enterocolitis (NEC). The etiology of these complications is multifactorial including diastolic retrograde mesenteric artery blood flow, prolonged intubation, and extensive aortic arch reconstruction. All of these concerning problems can occur during the initiation and advancement of enteral feeds. Lack of a coordinated approach to feeding infants with HLHS may increase the incidence of gastrointestinal morbidity and ultimately worsen outcomes. Methods: A multidisciplinary team developed an evidence based feeding protocol that comprehensively addresses all feeding issues from the prenatal period until the infant's second palliative surgery. The protocol begins with a prenatal parental visit with the feeding team to prepare the family. The protocol then proceeds to a feeding team consult when the infant is in the PICU pre-operatively. A nonnutritive oral stimulation plan is established. Following surgery a detailed algorithm outlines how to advance the feeds, or alternately, if the infant is not feeding well, a stepwise approach to addressing the gastrointestinal problems. An approach to managing necrotizing enterocolitis is also included in the protocol. Following discharge out-patient follow-up continues according to the Children's Hospital of Wisconsin guidelines with the feeding team's care coordinated with the Single Ventricle Clinic. For study purposes, all infants with HLHS, since the initiation of the protocol, were reviewed for feeding status and gastrointestinal complications. Abdominal radiographs were reviewed retrospectively for signs of NEC utilizing the modified 
Bell's staging criteria. Results: The immediate result has been a significant decrease in provider variability and improved communication in the nutritional treatment of infants with HLHS. The protocol allows parents to understand the complexities related to feeding and to participate in the advancement of oral feeds with their medical team. Since the implementation of the feeding protocol none of the patients with HLHS who survived to begin enteral feeds developed pneumatosis or pneumoperitineum. Of these patients, $38.5 \%$ had no abnormal bowel gas patterns on abdominal radiograph. The remaining $61.5 \%$ had mildly abnormal radiographic findings consistent with no greater than stage IIA modified Bell's staging criteria, with none progressing to a more serious stage. Following discharge, all patients after stage 1 palliation have had ongoing feeding evaluation by the feeding team in combination with the Single Ventricle Clinic. Of these patients over one half $(61 \%)$ have advanced to exclusively full oral feeds prior to their stage 2 palliative surgery. None of these patients were readmitted between discharge and stage 2 palliation due to adverse gastrointestinal complications. Conclusions: Defining specific feeding guidelines and beginning the protocol in the prenatal period can result in improved patient outcomes and team dynamics.

Correspondence to Coleen Miller: mille003@mc.duke.edu

\section{Evaluation of extracorporeal CPR (ECPR) simulation based programme used to identify systems issues and improve the ECPR process}

Cecilia St. George-Hyslop, Anne-Marie

Guerguerian, Lisa Davey, Colleen Gruenwald, David Edgell, Afrothite Kotsakis

\section{The Hospital for Sick Children, Toronto, Canada}

Background: Extracorporeal cardiopulmonary resuscitation (ECPR) is the rapid deployment of ECMO to rescue patients from refractory cardiac arrest. Time to rescue patients onto this technology is dependent on efficient performance of a large interdisciplinary team. Hypothesis: An ECPR simulation programme is feasible and can identify the systems issues that lead to error. Methods: Monthly simulated ECPR scenarios and formal debriefing sessions were run during the day in the Critical Care Unit. Simulations included traditional CPR, priming the ECMO circuit, choosing cannulae, blood being delivered and the circuit initiated. All members of the interdisciplinary team were involved; nurses, perfusionists, respiratory therapists, intensive care physicians, cardiovascular surgeons, social work, blood bank laboratory, paging services, unit clerks and ward aides. 8 individual focused interviews were conducted to identify system improvement themes. Results: A simulation based ECPR programme is feasible and endorsed by all members of the team. System errors were identified which resulted in changes to our ECPR process. 1. Workload errors: Team members' roles are explicitly defined. 2. Knowledge and experience errors: Simulation technology is used to educate all new CVS and CCU fellows on ECPR, ECMO and communication skills. The proper use of defibrillators is reviewed regularly. 4. Distraction errors: A 'physical space map' for people and equipment and a checklist to be used during ECPR were developed. Conclusions: Simulation is an effective tool for exploring and implementing system changes to improve an ECPR process that involves a large interdisciplinary team. Future goals include examining prospectively, the time needed to initiate ECMO during ECPR since changes to the process have been implemented. Due to the feasibility and success of this ECPR program, we are expanding the simulation program to include trauma, burn codes, and out of hospital ECMO transport.

Correspondence to Cecilia St. George-Hyslop: cecilia.hyslop@ sickkids.ca

\section{Malnutrition in infants undergoing the staged Norwood repair}

Sunita Ferns, Gia Spiotto, Andrew VanBergen, Michel Ilbawi, Tarek Husayni

The Heart Institute for Children, University of Ilinois at Chicago, Chicago, IL, USA

Background: Failure to thrive is a common problem in children undergoing the staged Norwood repair. The first interstage period is fraught with difficulties. Data looking at nutritional correlates in this group is limited. Methods: The purpose of this study was to evaluate risk factors for malnutrition in infants before the bidirectional Glenn procedure. Our study group included infants surviving the Stage 1 Norwood who went on to have the BDG in a tertiary cardiac centre. A retrospective chart note audit was conducted. Weight for age and length for age $z$ scores were used as an indicator of nutritional status. These were computed at birth, discharge and at admission for the bidirectional glenn procedure (BDG). Demographic data, perioperative, intraoperative details and post discharge information were collected. Results: 30 infants (16 males and 
14 females) were studied in a 26 month period. The median weight was $3.13 \mathrm{kgs}(2.2-4.12)$ with a median $\mathrm{z}$ scores of -0.21 ( -2.6 to 1.83$)$. The median age at surgery was 8.5 days (3-34). The median discharge weight was $3.3 \mathrm{kgs}(2.2-4.3)$ with a median $z$ score of $-1.29(-3.15$ to 0.85$)$ after a hospital stay of 32 days (17-64). 27 patients were on nasogastric (NG) feeds at discharge and the rest were fed orally, however at the BDG only 6 patients remained on NG feeds. 28 patients required caloric supplementation at discharge and 10 of these were on fortified breast milk. At discharge 12 patients were on antacids and 19 patients were on antireflux medications. These numbers were similar prior to the second stage. During the interstage period 13 patients required a rehospitalisation for a duration exceeding 48 hours. Five of these infants were hospitalized twice. The median weight at admission for BDG was $5.2 \mathrm{kgs}(3.47-7.72)$ with a median $\mathrm{z}$ score of -1.44 ( -3.2 to 1.32 ). Univariate correlates of weight for age $\mathrm{z}$ scores at the BDG were computed and then multiple linear regression analysis was performed with predictor variables that had a significant association on the univariate analysis. Infants with a lower $\mathrm{z}$ score at discharge, a longer bypass time, higher postoperative inotrope score, longer hospitalization duration and greater number of hospital readmission had a lower weight for age $z$ score at BDG $(p<0.05)$. Breast fed infants had a higher wt for a age $z$ score $(p<0.05)$. Infants with a lower $z$ score at discharge and greater number of readmissions had a lower weight for age $\mathrm{z}$ score at the $\mathrm{BDG}(\mathrm{R} 2=0.54)$ on a multiple linear regression model. Conclusions: Infants undergoing the staged Norwood procedure are at a risk for malnutrition. These infants have higher metabolic demands and are poor feeders. Factors contributing to this are complex however we noted difficulties during the postoperative and interstage period as significant contributors. Poor physical growth in infancy may have implications for future growth and development and this should therefore be aggressively treated.

Correspondence to Sunita Ferns: sjulianaferns@yahoo.com

\section{Surgical Infection Prevention Collaborative}

Gail Keyser, Eva Teszner, Eileen Brunwasser

Childrens Hospital of Philadelphia, Philadelphia, PA, USA

Background: The purpose of the Collaborative is to improve the quality of care delivered to pediatric patients who undergo surgery through the application of evidence-based surgical infection prevention practices before, during and after the surgical procedure. The CHOP team chose to target the population of patients undergoing a primary $\mathrm{CT}$ surgery through a mediastinal incision, and to limit the test practices to those that are better supported in the pediatric literature: 1. Selection, timing, intra-operative re-dosing and post-operative discontinuation of surgical prophylactic antibiotics. 2. Appropriate hair removal and skin antisepsis before surgery. 3. Basic surgical infection prevention strategies. Methods: A multidisciplinary team was convened and retrospective data was collected to establish practice baselines. A concurrent data collection system was developed and implemented and new practices were introduced, and when possible, embedded into redundant systems to prevent failures. A multidisciplinary antibiotic guideline was also developed. The data shows that the strategies used were effective in producing practice changes during the 6 month collaborative period. Results: Implementation and assessment of appropriate use of prophylactic antibiotics as follows: Selection: $95 \%$ of patients receive prophylactic antibiotics consistent with hospital guidelines. Start Time: $95 \%$ of patients receive antibiotic prophylaxis initiated $0-120$ minutes before the incision. Re-dosing: 95\% of patients for whom re-dosing is indicated receive a second dose of Intra-operative antibiotics consistent with hospital guide lines. Post-operative discontinuation: $95 \%$ of patients have prophylactic antibiotics discontinued after 24 hrs. Conclusions: The data shows that the strategies used were effective in producing practice changes during the 6 month collaborative period. For the purpose of this presentation, the integral role and influence of the CT OR nurses is emphasized. A multi disciplinary antibiotic guideline was also developed.

Correspondence to Gail Keyser: keyser@email.chop.edu

First trimester and early second trimester diagnosis of fetal tetralogy of Fallot: diagnostic yield and accuracy

Aarti Bhat, Devin W. Kehl, Theresa Tacy, Michael Brook, Lisa K. Hornberger

University of California San Francisco, San Francisco, CA, USA

Background: Fetal echocardiography is a well-established modality for detailed assessment of fetal heart 
disease (FHD). Its use, however, is largely reserved for pregnancies $>17$ weeks gestation. While the prenatal detection of fetal heart disease at $<17$ wks has been demonstrated, no study to date has elaborated on the extent of anatomic detail in early fetal echo exam in the presence of FHD. Methods: We compared detail of anatomic visualization before (Group I) and after (Group II) 17 weeks gestation in fetal tetralogy of Fallot (fTOF). Detailed 2D, color and pulsed Doppler fetal echo assessment were performed and retrospectively analyzed on a 10 point scoring system assigned to every component other than ventricular septal defect and overriding aorta to quantitatively interpret anatomical detail; 1 point each was ascribed to complete and unequivocal visualization of pulmonary stenosis (PS), pulmonary valve $(\mathrm{PV})$, pulmonary arteries including dimensions (PA), PA flow, systemic veins, pulmonary vein (PV), atrioventricular valve, duct, ductal direction/flow, aortic arch and sidedness. Results: There were 10 cases in Group I [12 weeks (2), 14 weeks (5), and 17 weeks (3)] and 25 cases in Group II. Referral for Group I was for extracardiac pathology (80\%) [Omphalocele (2), ectopia cordis (2), trisomy 21 (1), triplets (1)] and suspected FHD in Group II (64\%). In Group I transabdominal approach was sufficient in 5, transvaginal approach was diagnostic in 3 and contributory in 2 cases. No cases in Group II needed transvaginal study. Chromosomal anomalies were found in $1(10 \%)$ of Group I and 9 of Group II $(36 \%)$. Of 10 points for individual anatomical details, Group I had a mean score of 6.1 (range 2.5-9, median 7.5) versus 8.4 (range 6.5-10, median 9) in Group II. Ductal anatomy including size, direction of flow and aortic arch anatomy including sidedness were almost equally well seen in both groups. PA and PV were reliably identified in $45 \%$ and $20 \%$ in Group I as compared to $86 \%$ and $90 \%$ in group II. However, almost all these group I cases had significant anterior abdominal wall defects or $\mathrm{CDH}$. Of Group I, 8 had pregnancy termination (2 after a mid trimester fetal echo, 2 with autopsy confirmation), 1 had late fetal demise, one was liveborn with postnatal confirmation. In Group II, of 24 with follow-up, 7 had pregnancy termination, 2 had fetal demise, 12 delivered with confirmed postnatal anatomy and 3 are awaiting delivery. Conclusions: Our semi-quantitative comparative analysis in fTOF indicates that not only can a basic anatomic diagnosis be made in the first and early second trimester, but, a lot of the anatomic nuances as well as quantitative measurements can be reliably undertaken in this group. The higher incidence of co-existing cardiothoracic pathologies may have contributed to poorer PA and PV visualization in Group I. Indications for referral and co-existing fetal pathology may well direct the timing of the first fetal echo, but even this early study can be detailed and accurate enough to guide management.

Correspondence to Aarti Bhat: hejmadia@sbcglobal.net

\section{Associated cardiac comorbidities in surviving adult patients with cardiac segments $\{S, L, L\}$ do not influence the progression of disease or diminish quality of life}

Richard Donner, Martin St. John Sutton, Paul Weinberg, Gary Webb

\section{Children's Hospital of Philadelphia, Hospital of the} University of Pennsylvania, Philadelphia, PA, USA

Background: Characteristics of adult patients with congenitally corrected transposition of the great arteries (CCTGA) have been studied widely. Less is known of the larger group of patients with cardiac segments $\{S, L, L\}$, defined as situs solitus of viscera and atria, ventricular L-loop and L-transposed great arteries (aorta arising from the RV or its remnant and pulmonary artery from the LV). Methods: We examined the most recent records of a 35 patient cohort with cardiac segments $\{\mathrm{S}, \mathrm{L}, \mathrm{L}\}$ (ages 19-62, median 33 years) for previous or existing comorbidities including associated anomalies, related consequences and interventions. These included the presence of heart block, ventricular septal defect, pulmonary stenosis, tricuspid regurgitation, diminished ventricular function, double inlet left ventricle, left sided tricuspid atresia, atrial ectopy, and previous palliative, open heart or Fontan surgery. NYHA class, employment and educational status were also noted as measures of quality of life (QOL). Patients were assigned to a younger group (group 1) or older group (group 2) and the prevalence of individual comorbidities between the groups was tested using the chi square statistic and distribution. Results: Eighteen patients were male and 17 female with median ages equal to that of the cohort. Twenty patients were less than or equal to the median age (group 1) and 15 patients were greater than the median age (group 2). With the exception of VSD $(p=0.03)$, the proportion of each potentially adverse comorbidity, including double inlet LV, left sided tricuspid atresia and Fontan surgery, was not significantly different in the two groups $(p \geqslant 0.14)$. QOL measures were very encouraging. The proportion of each adverse QOL measure was also not significantly different in the two groups $(\mathrm{p} \geqslant 0.12)$. Conclusions: (1) The similarity of comorbidities in 
groups 1 and 2 argues against progression of disease that would reduce their prevalence in group 2. (2) In the same way, the similarity of adverse QOL measures in the two groups suggests that QOL is maintained with advancing age.

Correspondence to Richard Donner: donnerr@email.chop.edu

Is there an optimal time for the completion of total cavopulmonary connection after bidirectional Glenn anastomosis?

Naira Mkrtchyan, Christine Karapetyan, Liana Hakobyan, Irina Bakunts, Hagop Hovaguimian, Karen Kurdov

Nork Marash Medical Center, Heart Clinic, Yerevan, Armenia

Background: This retrospective study aims to explore whether there is a safe maximum waiting period for completion of TCPC (total cavopulmonary connection) after bidirectional Glenn (BDG) anastomosis. Methods: This study includes 42 patients, who had 2 or more years follow-up following BDG. Our policy has been to perform TCPC only, when we had arterial oxygen saturation $\left(\mathrm{SaO}_{2}\right)$ deterioration and/or parental dissatisfaction of their child's health. We assessed $\mathrm{SaO}_{2}$, physical growth, parental assessment of child's health, ventricular function, AV valve regurgitation, and systemic ventricle volume index data immediately after the operation, after 2 years (usual TCPC completion period) and during the last visit. Results: The mean follow-up of the 42 patients was 66.2130 (24-147) months. $\mathrm{SaO}_{2}$, systemic ventricular ejection fraction and systemic ventricle volume index remained unaffected during the follow-up period. There has been improvement of physical growth estimated by weight $(\mathrm{p}<0.001)$ and height $(\mathrm{p}<0.0001)$ percentiles. $40(95 \%)$ of the parents assessed their child's overall health as satisfactory and $2(5 \%)$ as unsatisfactory in early post operational period, and remained $32(80 \%)$ and $8(20 \%)$ respectively in last visit. There has been statistically significant increase of $\mathrm{AV}$ valve regurgitation by 0.3 grade $(\mathrm{p}<0.008)$. At an average of 63.6118 (24-99.6) months after BDG 17 patients underwent completion to TCPC. There was no mortality or complication after TCPC completion. Conclusions: If we choose to, it may be safe to wait up to 5 years, before performing completion TCPC following $\mathrm{BDG}$, provided $\mathrm{AV}$ valve regurgitation is followed closely.

Correspondence to Naira Mkrtchyan: mknava2000@yahoo.com
Large left and right ventricular tumors: survival after management as univentricular heart utilizing a hybrid interventional and surgical approach

Grace Freire, Andrew Fiore, Kenneth Schowengerdt, Saadeh Jureidini

Saint Louis University and Cardinal Glennon Children's Medical Center, St Louis, MO, USA

Background: Management of neonates with large cardiac tumors resulting in univentricular heart physiology has been directed at total or subtotal tumor excision or a Norwood-like palliation procedure. The results are discouraging due to complications such as formation of subaortic fibrous ridge, postoperative arrhythmia, recurrence of the tumor and neonatal death. We report on the shortterm survival after the innovative use of the hybrid approach for 2 neonates with large left ventricular tumors and a Blalock-Taussig (B-T) shunt for a large right ventricular tumor. Methods: The 3 patients were diagnosed prenatally, during the second trimester, with large obstructive cardiac tumors. The tumor size ranged from $27 \mathrm{~mm}$ to $38 \mathrm{~mm}$ in the largest diameter. In one patient the mass doubled in size over a 2 week span. Two patients had evidence of heart failure, were treated with digoxin and improved. Two patients had large tumors occupying the majority of the left ventricle and causing decreased antegrade flow across the aorta, bidirectional ductal flow and reversal of flow via the foramen ovale. Both had severe mitral regurgitation and decreased systolic function, cardiomegaly and pericardial effusion. At birth, ductal patency was maintained with prostaglandin infusion until 1 week of age when a hybrid procedure was performed consisting of bilateral banding of the pulmonary arteries and stenting of the ductus arteriosus. One week later static balloon dilatation of the foramen ovale was performed without complications. The third patient was diagnosed at 33 weeks of gestation with a large right ventricular tumor obstructing the right ventricle with 4 additional smaller left ventricular tumors also noted. There was right atrial dilatation and atrial flutter with variable conduction. The tricuspid valve leaflets were not identified as they were covered with the tumor. There was severe mitral regurgitation, retrograde flow in the aortic and ductal arch, pulmonary insufficiency, pleural effusion and ascitis. After birth the patient was started on prostaglandin and successful cardioversion was achieved after amiodarone and propafenone were loaded. Results: The 2 patients with LVOT obstructing tumor were 
discharged home at 4 weeks of age and are doing well. The 3rd patient underwent B-T shunt placement at 1 week of age and was sent home after 3 weeks, arrhythmia-free, in stable condition. None of the patients had evidence of heart failure, and there was no morbidity or mortality. Conclusions: We conclude that: 1) The Hybrid approach for large left ventricular tumors should be considered as treatment of first choice. 2) Management of large right-sided tumors is tailored so that adequate pulmonary flow is established and arrhythmia controlled. 3) In order to avoid the reported high mortality risk, surgical interventions such as conventional Norwood procedure and tumor resection or biopsy are not recommended.

Correspondence to Grace Freire: gfreire@slu.edu

\section{Creating an environment that supports nursing research in a pediatric cardiac center}

Ashley Elford, J-S Rim, Sharon Barton, Paula Cho, Jennifer Poserina, Rachel Ryan, Mary Weldon, Michelle Ulrich

The Children's Hospital of Philadelphia, Philadelphia, PA, USA

Background: In the past, research utilization at our institution was organized around creating policies and procedures. Bedside nurses were involved, yet had little input into setting priorities for aspects of care that required updating using evidence based practice. Methods: Shared governance structures were implemented in 2004. Nursing research was decentralized into unit and hospital based committees. Granger and Chulay's "Research Strategies for Clinicians" was utilized to provide concrete processes for developing the committee and structuring projects. Restructuring offered the opportunity for nurse involvement; however, similar to other institutions, supports and tools for committees were not fully developed. Nurses reported fear about research methods, especially statistics. Other barriers included time limitations, lack of knowledge and resources, and little understanding about how research effects practice. Recommendations for improvement included developing research skills, identification of research topics, "making research fun", and maximizing resources. Strategies to create a culture for nursing research included creation of a unit-based nursing research committee, consolidation into a Cardiac Center Committee, involvement with a research committee in the PICU, participation in a joint Cardiac Center/PICU research study, development of an introductory tool to introduce nurses to research methods, and introducing research during nursing orientation. Resources to the committee include medically experienced clinical nurse researchers. Committee members are involved in ongoing recruitment. Three projects were implemented: a capnometry study to verify NG tube placement (joint study with PICU), a 'chocolate chip' study to introduce research concepts, an evaluation of the nurse call system to evaluate data collection and outcome reporting. Results: Members met monthly and reported good communication from meetings in which they were unable to attend. Nurses participating in the collaborative capnometry study reported an increased knowledge of research methods. Members learned pitfalls of clinical nursing research. Future contacts and hospital research resources were identified. 107 staff participated in the chocolate chip study, during which participants learned steps in conducting research, created a fun atmosphere for research, learned how to develop and critique research, learned the IRB process, and involved a multidisciplinary team. Finally, standard research methods were used to investigate the Cardiac Center use of the nurse call system. The data identified situations where the nurse call paging did not improve patient safety when compared to the prior system. Conclusions: In eight months, our group has successfully developed a nursing research committee, with collaborative and single site projects completed. Goals for the future include prioritizing and developing a research study, and creating opportunities for nursing contributions in centerwide multidisciplinary research.

Correspondence to Ashley Elford: elford@email.chop.edu

\section{A pilot orientation program}

Ann Adams, Karen Goldschmidt, Linda Goss, Cara Rakow, Lorri Rudolph, Amanda Seelhorst

Children's Hospital of Philadelphia, Philadelphia, PA, USA

Background: Beginning a nursing career in a critical care environment can be a complicated and overwhelming for the graduate nurse. New nurses need to learn basic assessment skills as well as develop a system of time management. These tasks are difficult to learn in a complex environment of an 
intensive care unit with critically ill patients whose care changes rapidly. Sensitive to the challenges that graduate nurse face, the Cardiac Center developed a comprehensive orientation program. This program was developed to incorporate a variety of unique learning experiences allowing the novice to systematically progress in the development of clinical skills, critical thinking and professional development. Methods: Recognizing that each nurse within the institution has a unique skill set, and that learning from one another contributes to the success of building a strong foundation, opportunities were arranged for graduate nurses to practice with nurses in other areas within the institution. Prior to beginning their orientation in the Cardiac Center, novice nurses worked alongside preceptors in subacute units (satellite units) for 4 weeks. This experience enabled the graduate nurse to be exposed to a wide variety of patient acuities across the continuum of care. Success Factors $\square$ Creating a sense of connection with the Cardiac Center $\square$ Daily check-ins by Cardiac Center Orientation Coordinator $\square$ New Staff Weekly Support Group Bi-weekly Cardiac Education $\square$ Assignment of a Cardiac Center Mentor. Results: To demonstrate program outcomes participant surveys were conducted with orientees, preceptors and orientation coordinators on both units. Results of these surveys will be shared in this poster presentation. Overall survey results found that graduate nurses involved in the pilot orientation program were more confident and arrived at the Cardiac Center with a solid knowledge base in assessment, organization and prioritization skills. A sense of connection was maintained with the Cardiac Center through the various programs that were put into place and worked to retain the graduate nurses until they began orientation in the Cardiac Center. Length of orientation was not a factor affected by the pilot program. Conclusions: The Cardiac Center developed a comprehensive orientation program that incorporates a variety of unique learning experiences allowing the novice to systematically progress in the development of clinical skills, critical thinking and professional development. Prior to beginning their orientation in the Cardiac Center, novice nurses work alongside preceptors in sub-acute units (satellite units) for 4 weeks. This experience exposes the novice nurse to a wide variety of patient acuities. Graduate nurse involved in the pilot orientation program are more confident and arrive at the Cardiac Center with a solid knowledge base in assessment, organization and skill prioritization.

Correspondence to Ann Adams: adamsa@email.chop.edu
Superior mesenteric artery blood flow and response to enteral feeding in neonates with congenital heart disease: Doppler evaluation and abdominal oximetry

Christina Phelps, Robert H. Friesen,

Regina M. Reynolds, Eduardo da Cruz, Jon Kaufman

\section{The Children's Hospital and University of Colorado Health Science Center, Denver, CO, USA}

Background: The splanchnic circulation is vulnerable in neonates with congenital heart disease and decreased gut perfusion may complicate the perioperative course. Oxygen delivery may be impaired due to overall low cardiac output or be compromised by specific cardiac lesions, vasoactive medications, or cardiopulmonary bypass. Impaired oxygen utilization may also occur and the effect and demands of enteral feeding on the splanchnic circulation in these patients is unknown. Regional, site specific monitoring of oxygen saturation $\left(\mathrm{rSO}_{2}\right)$ with near infrared spectroscopy (NIRS) could prove helpful in the peri-operative period in terms of assessing adequacy of splanchnic blood flow and determining the timing and safety of initiating enteral feeds. Little is known about the characteristics of superior mesenteric artery (SMA) flow and splanchnic perfusion in this population. However, abnormal SMA Doppler indices have been associated with an increased risk of necrotizing enterocolitis in neonates. We present our preliminary findings detailing SMA blood flow indices and splanchnic $\mathrm{rSO}_{2}$ in twelve neonates with various congenital heart lesions in the peri-operative period. Methods: After Colorado Multi-institutional review board approval and informed consent, 12 neonates admitted to the Cardiac Intensive Care Unit (CICU) with congenital heart disease were studied pre and eight hours post-operatively and immediately before and 1 hour after the initiation of enteral feeds. Baseline studies of abdominal site NIRS and SMA echocardiographic flow patterns were obtained. The indices measured included peak systolic velocity (PSV), timed average mean velocity (TAMV), enddiastolic velocity (EDV). Resistive index (RI), and pulsatility index (PI) were calculated. Once enteral feeds started, SMA blood flow response was measured simultaneously by Doppler and NIRS. Results: Twelve infants with congenital heart disease were included in this study. Three had single ventricle physiology both pre and postoperatively (one did not survive to the feeding stage of the study). 8/12 patients demonstrated an increase in the $\mathrm{rSO}_{2}$ post-operatively. During simultaneous 
SMA Doppler evaluation 7/12 demonstrated a decrease in the PSV, 11/12 had decreased/unchanged RI and 9/12 had decreased PI. In response to feeding, 9/11 infants had reduced/unchanged splanchnic $\mathrm{rSO}_{2}(2 / 11$ had a significant increase in $\left.\mathrm{rSO}_{2}\right), 8 / 11$ demonstrated an increase in the PSV, 6/11 had decreased/unchanged RI, and 7/11 had decreased/unchanged PI. Conclusions: This is the first study to report postoperative intestinal flow patterns and splanchnic $\mathrm{rSO}_{2}$ response to the demands of enteral feedings in neonates with congenital heart disease. Doppler flow indices of the SMA in these twelve infants with congenital heart disease demonstrated variable responses to initiation of feedings with a trend towards increased peak systolic velocity and decreased resistance indices. Regional saturations as measured by NIRS over the anterior abdominal wall did trend towards a reduction in $\mathrm{rSO}_{2}$ of the splanchnic bed at one hour post-feeding. The clinical significance of our findings is that enteral feeding may be associated with a physiologic decrease in splanchnic $\mathrm{rSO}_{2}$ (possibly due to increased oxygen utilization by the gut) with a simultaneous compensatory increase in SMA flow velocity and alteration in resistive indices. Further studies are required in this population and in normal controls in order to confirm this hypothesis.

Correspondence to Christina Phelps: crisree@aol.com

\section{Survival after extracorporeal cardiopulmonary resuscitation in infants and children with heart disease}

Titus Chan, Susan Bratton

Primary Children's Medical Center, University of Utah, Salt Lake City, UT, USA

Background: Survival following CPR has variable results with recent reports of hospital survival as low as $12 \%$. There has been an emerging interest in the use of extracorporeal membrane oxygenation (ECMO) as a rescue technique (ECPR) for patients in whom conventional $\mathrm{CPR}$ has not reestablished a perfusing rhythm. Patients with congenital heart disease are the largest proportion of patients treated with ECPR and often have a reversible cause for their cardiac arrest. These children may have increased survival using ECMO support if the underlying reason for the cardiac arrest is adequately treated. There has been little research examining the impact of specific cardiac physiology and recent congenital heart surgery on the outcomes of ECMO used as ECPR. In this study, we utilized data from children with heart disease who received ECPR as reported to the Extracorporeal Life Support Registry (ELSO). Our goal was to determine if outcomes for these children were associated with patient demographics, pre-ECMO management, type of cardiac disease and ECMO support variables. Methods: Children $(<18$ years) with heart disease who received extracorporeal cardiopulmonary resuscitation reported to the Extracorporeal Life Support Organization database were evaluated. Patients were classified into four groups based on underlying cardiac physiology: single ventricle, two ventricles, cardiac muscle disease, and pulmonary hypertension. Patients with eligible procedure codes were assigned a Risk Adjustment for Congenital Heart Surgery-1 (RACHS-1) classification. Pre-ECMO demographics, support details, cardiac diagnostic groups and RACHS-1 groups and ECMO complications were evaluated for an association with hospital survival. From this, several multivariable logistic regression models were constructed to evaluate independent associations with hospital survival. Results: Use of extracorporeal membrane oxygenation as an adjunct to cardiopulmonary resuscitation resulted in hospital survival in $42 \%$ of infants and children with heart disease. Single ventricle patients had a higher mortality (64\%) compared to those with two ventricles (52\%). Higher RACHS-1 categories were associated with lower rates of survival among subjects for whom a RACHS-1 category could be assigned. In the regression models, single ventricle physiology (Odds Ratio 1.6, 95\% Confidence Interval 1.05-2.4) as well as a history of a Stage 1 type procedure (Odds Ratio 2.7, 95\% Confidence Interval 1.2-6.2) was associated with increased mortality. Prior to ECMO, extreme acidosis $(\mathrm{pH}<7.01)$ was associated with increased mortality (Odds Ratio 2.2, 95\% Confidence Interval 1.3-3.7) while right carotid artery cannulation was associated with decreased mortality (Odds Ratio 0.6, 95\% Confidence Interval 0.4-0.9). Once on ECMO, complications such as renal failure, evidence of neurological injury on radiological imaging, and persistent acidosis were associated with increased hospital mortality. Conclusions: Use of extracorporeal membrane oxygenation as an adjunct to cardiopulmonary resuscitation resulted in hospital survival in $42 \%$ of infants and children with heart disease. Underlying cardiac physiology and associated cardiac surgical procedures influenced mortality as did pre-extracorporeal resuscitation status, and ECMO associated complications.

Correspondence to Titus Chan: tituschan@gmail.com 
Ketamine as a neuroprotective agent in children undergoing cardiac surgery: results of magnetic resonance spectroscopy

Adnan Bhutta, K. J. S. Anand, Michael L. Schmitz, Diana Lindquist

University of Arkansas for Medical Sciences, Little Rock, $A R$ and Cincinnati Children's Hospital Medical Center, Cincinnati, OH, USA

Background: Cardiopulmonary bypass (CPB) may lead to neurologic morbidity with long-term cognitive decline in children undergoing cardiac surgery. Ischemia, infarction, or endothelial activation may promote glutamate excitotoxicity. Neonates and infants are more susceptible to excitotoxic cell death and glutamate receptor antagonists potentially can prevent this damage. Ketamine blocks N-Methyl D-Aspartate (NMDA) receptors and has anti-inflammatory effects. We investigated if ketamine would prevent changes in the apparent diffusion coefficient (ADC) or MRS measured-metabolite levels in children undergoing CPB. Methods: 10 children under 1 year of age undergoing surgery to repair VSD had MRI/MRS performed prior to surgery and again prior to hospital discharge. Five infants received ketamine ( $2 \mathrm{mg} / \mathrm{kg}$ iv) just before CPB [cases]; the other five received saline [controls]. All children were brought to the MRI suite, anesthetized, and scanned on the day of surgery. They were then transported to the cardiovascular surgical suite for their operation. Four days after surgery, the infants were brought back to the MRI suite, anesthetized, and re-scanned. All data were acquired with a 1.5T GE LX system. Localizer images were acquired, followed by dual-echo proton density/T2-weighted images covering the entire brain. Diffusion-weighted images were acquired to calculate the apparent diffusion coefficient. PRESS single voxel spectra were acquired in frontal white matter (FWM) and basal ganglia (BG) at TE's of 35 and $144 \mathrm{~ms}$ and TR $2500 \mathrm{~ms}$. ADC maps were calculated using vendor software. Metabolite concentrations were estimated with LCModel and analyzed using SPSS 13 for Windows. Results: The median age of both groups was 5 months and both groups included 4 females and 1 male. Mean CPB time for cases was 63.2 minutes while the mean CPB time for controls was 57.8 minutes $(p=0.59)$. No significant differences in ADC occurred between treatment groups or between pre- and post-surgery scans. TE 35 data: Creatine $(\mathrm{Cr})$ levels increased significantly between the pre- and post-surgery scans in the control group in BG $(p=0.02)$. Glutamate + glutamine (Glx) concentrations were significantly lower for the ketamine group vs. the control group $(\mathrm{p}=0.02)$ following CPB surgery in the FWM. Glx decreased significantly in the ketamine group between pre- and post-surgery $(\mathrm{p}=0.02)$ measurements. TE 144 data: Choline (Cho) was decreased significantly in BG and FWM for the ketamine group post-surgery $(\mathrm{p}<$ 0.03 ), whereas a similar trend occurred for FWM Cho levels in the controls in $(\mathrm{p}<0.1)$. Conclusions: Ketamine decreased Glx levels in the FWM compared with the control group post-surgery suggesting that the NMDA blockade by ketamine decreases glutamate release. As glutamate-mediated excitotoxicity is one of the purported mechanisms of CNS injury after $\mathrm{CPB}$, lower glutamate levels could lead to increased neuronal survival in these areas, ultimately benefiting the cognitive outcomes of ketamine-treated children. The decreased Cho is probably related to changes in acetylcholine (ACh) levels (ketamine also blocks ACh receptors) or membrane metabolism/composition resulting from the neurological insult. Elevated BG $\mathrm{Cr}$ in controls suggests changes in energy metabolism in response to the surgery. This increase was not present in the ketamine group suggesting that ketamine may reduce energy consumption in the basal ganglia.

Correspondence to Adnan Bhutta: bhuttaadnant@uams.edu

Factors associated with arch reintervention and growth of the aortic arch following coarctation repair in neonates weighing less than 2.5 kilograms

Tara Karamlou, Alessandra Bernasconi, Edgar Jaeggi, William G. Wiiliams, John G. Coles, Glen S. VanArsdell, Christopher A. Caldarone

Oregon Health and Science University, Hospital for Sick Children, Portland, OR, USA

Background: Neonates weighing less that $2.5 \mathrm{~kg}$ with aortic coarctation offer unique clinical challenges. We sought to determine the prevalence of death or aortic arch reintervention and their determinants after repair of aortic coarctation (CoA). In addition, we also sought to define growth trajectories for post-repair aortic arch dimensions and to identify factors associated with accelerated longitudinal growth. Methods: Retrospective review of neonates $<2.5 \mathrm{~kg}$ undergoing $\mathrm{CoA}$ repair between 1993-2004 was performed and competing-risks methodology used to determine timerelated prevalence of three mutually exclusive endstates: death, arch reintervention, and survival without subsequent reintervention. Serial echo data $(n=135)$ were collected over a median interval of 
9 months (range 0 to 5.4 yrs) after CoA repair with a median of 4 (range 1 to 11) measurements per patient. Mixed linear regression analysis was used to model longitudinal growth trajectories of aortic arch dimensions. Results: 36 neonates underwent CoA repair. Initial repair type was simple end-toend (SEE) $(n=3)$, extended end-to-end (EEE) $(n=16)$, subclavian flap aortoplasty $(n=15)$ and patch aortoplasty $(n=2)$. Median age at initial repair was 11 days (range 2-69 days) and mean weight was $2.0110 .33 \mathrm{~kg}$. Median initial aortic valve $Z$-score $(A V Z)$ was -1.1 (range -5.7 to 2.5 ), median initial transverse aortic arch Z-score (TAAZ) was -2.8 (range -9.5 to 0.3 ), and median initial aortic isthmus Z-score (ISTZ) was -3.9 (range -14.1 to -0.2 ). Overall survival at 1 year was $76 \%$. Competing-risks analysis predicted that after 1 year from initial repair 19\% had died without subsequent reintervention, $14 \%$ underwent arch reintervention, and $67 \%$ remained alive without arch reintervention. Risk-factors associated with arch reintervention included the presence of a VSD $(p=0.06)$. Serial echo data demonstrated that neonates with EEE repairs had increased TAAZ scores $(p=0.009)$. Although patients with larger initial TAAZ had higher TAAZ across all time points $(\mathrm{p}<0.001)$, neonates with the smallest TAA Z-scores (those in the bottom 25th percentile) had accelerated growth trajectories $(\mathrm{p}<0.001)$. Growth of the aortic isthmus was likewise accelerated in neonates with the smallest initial ISTZ $(\mathrm{p}<0.001)$ and those undergoing either patch augmentation or EEE repair $(\mathrm{p}<0.001)$. Conclusions: Mortality and arch reintervention are common after initial CoA repair in neonates $<2.5 \mathrm{~kg}$. Catch up growth of both the transverse arch and the isthmus occurs following CoA repair, especially in those with the smallest arch parameters, and can be increased by using extended end-to-end repair technique.

Correspondence to Tara Karamlou: karamlou@ohsu.edu

Safe and sound: improving performance by adapting principles learned of the aviation industry

Kristi Ryan, Kshitij Mistry, Jane Mericle, Michael Alton, James Jaggers, Karen Frush, Jon Meliones

\section{Duke Children's, Durham, NC, USA}

Background: Communication between teams has been shown to significantly affect patient care and safety. The Pediatric Critical Care Unit (PCCU) is an area of extreme vulnerability as patients are very complex, exhibit a greater vulnerability to human error due to high acuity, and require more frequent interventions. The airline industry has utilized crew resource management techniques including: briefings, check lists and scripted communication to reduce errors and improve team dynamics. Methods: Our Team Training model consisted of applying the aviation industry's approach to teamwork in a healthcare environment. Initial baseline data was obtained via Teamwork \& Safety Climate Surveys. In addition, an independent observer evaluated each work environment. Team Training consisted of an interactive curriculum, including: didactic lectures, "hands-on" demonstrations, "role playing," open discussions and training internal coaches. Each staff member underwent a 3-hour Team Training session. The initiatives that were personalized \& instituted include: critical language - "I need clarity" and "Is the attending aware", standardized communication utilizing SBAR (Situation, Background, Assessment and Recommendations) format, several pre- and post rounds briefings and huddles, standardized data sets for communication, and a "sterile cockpit" model. After completing training, a follow-up survey was administered and teamwork observed. Survey and observational data were compared using the Fisher exact test. Results: Surveys demonstrated improvement in how the staff perceived teamwork $(67 \%$ to $87 \%, \mathrm{p}=0.011)$, the use of briefings $(63 \%$ to $84 \%, \mathrm{p}=0.012)$ and knowing the proper safety channels $(67 \%$ to $87 \%, \mathrm{p}=0.007)$. Independent evaluations of staff performance showed an increase in overall teamwork by $72 \%(\mathrm{p}<0.001)$ and leadership perception $75 \% \quad(p=0.001) .95 \%$ of participants believed that team training would improve care and $100 \%$ would recommend team training. Conclusions: Team Training is essential not only for safe patient care, but also for team culture. Techniques used in the aviation industry are translatable to medicine but require "personalization" to each institution's specific culture and challenges. A structured approach to team training can result in significant improvements in team dynamics.

Correspondence to Kristi Ryan: kristi.ryan@duke.edu

\section{Age-dependent effect of Milrinone and Levosimendan on left ventricular contractility}

Janus Adler Hyldebrandt, Signe Holm Larsen, Michael Rahbek Schmidt, Vibeke Hjortdal, Hanne Ravn

Aarbus University Hospital - Skejby, Aarbus N, Denmark 
Background: Use of inodilators in postoperative care for neonates following cardiac surgery has largely been based on experience from adults. So far age-dependent response to inodilators has only been sparsely investigated. The myocardium matures throughout infancy although the exact time when myocytes are fully developed is unknown. We therefore designed an animal study where the hemodynamic responses to two inodilators were investigated in newborn and older hearts. In the present study, the effect of Levosimendan (Simdax ${ }^{\circledR}$, Abbott Scandinavia AB, Solna, Sweden) a calcium sensitizer and Milrinone (Corotrop $^{\mathbb{B}}$, Sanofi Synthlabo, Bromma, Sweden) a PDE III inhibitor was investigated. Our primary endpoint was change in contractility in the left ventricle as judged by pressure-volume-loops derived by the conductance catheter technique. Methods: Forty-nine piglets, either 4-6 weeks $(\mathrm{n}=24)$ or newborn $(\mathrm{n}=25)$ (Mean: 5.6 days SD1.7) were anesthetised and instrumented with conductance and pulmonary artery catheters, balloon catheter in the IVC, and paced internally by atrial pacing. Two clinically relevant infusion rates of each product were used to reach serum levels in the high and low end respectively, of the therapeutic range. Milrinone: Loading bolus of $50 \mu \mathrm{g} / \mathrm{kg}$ followed by infusion of $0.3 \mu \mathrm{g} / \mathrm{kg} / \mathrm{min}$. A second dose of $75 \mu \mathrm{g} / \mathrm{kg}$ at $60 \mathrm{~min}$ followed by infusion of $0.6 \mu \mathrm{g} / \mathrm{kg} / \mathrm{min}$. Levosimendan: Loading bolus of $12 \mu \mathrm{g} / \mathrm{kg}$ followed by $0.1 \mu \mathrm{g} /$ $\mathrm{kg} / \mathrm{min}$. A second loading dose of $24 \mu \mathrm{g} / \mathrm{kg}$ at $60 \mathrm{~min}$ followed by $0.2 \mu \mathrm{g} / \mathrm{kg} / \mathrm{min}$. Pressure development over time ( $\mathrm{dP} / \mathrm{dTmax})$ was measured in paced steady state, during expiration. By using the conductance technique we measured preload recruitable stroke work (PRSW) a pre- and afterload independent measurement of contractility. Data are presented as relative changes to placebo for each treatment group (CI95\%). Results: High dose Milrinone produced a $23.9 \%(1.49-1.03)(\mathrm{p}<0.005)$ increase in $\mathrm{dP} / \mathrm{dtmax}$ in the older hearts compared to placebo, but only a $10.5 \%(0.88-1.38)$ increase in the newborn hearts (NS). Levosimendan produced a $16.8 \%$ (1.01-1.36) $(\mathrm{p}<0.05)$ increase in the older hearts but a $9.9 \%$ $(0.73-1.14)$ decrease in the immature group (NS). In accordance with these results, high doses of Milrinone and Levosimendan produced the following changes in PRSW respectively: Milrinone: Older: $25.7 \%$ (0.90-1.76) increase. Newborn: $28.9 \%(0.47-1.10)$ decrease. Levosimendan: Older: $3.3 \%$ (0.73-1.46) increase, Newborn: $28.0 \% \quad(0.48-1.17)$ decrease. Neither low dose Milrinone nor Levosimendan produced any significant changes. Conclusions: Both drugs increased $\mathrm{dP} / \mathrm{dTm}$ ax significantly in the mature hearts but had lower or negative effect in the immature hearts. Both drugs produced a negative effect on PRSW in the immature hearts. Levosimendan had a negative effect on both $\mathrm{dP} / \mathrm{dtmax}$ and PRSW in the immature hearts. Further studies are needed to identify the effect of inodilators on immature hearts. Comparison of PDE III activity in mature and immature hearts, and binding of Levosimendan to troponin in the immature heart, are needed to understand these findings completely.

Correspondence to Janus Adler Hyldebrandt: jahy@studmed.au.dk

\section{Ideal enoxaparin dosing and monitoring in pediatric cardiac patients}

Cynthia Barclay, Catherine Dent, Larry Markham

\section{Cincinnati Children's Hospital Medical Center, Cincinnati, OH, USA}

Background: Children with congenital and acquired heart disease are at an increased risk for thrombus formation for several reasons, including low cardiac output, dilated heart chambers, intracardiac shunts, atrial dysrhythmias, and presence of synthetic material. When oral anticoagulation is unsafe or impossible, enoxaparin is often used. Standard enoxaparin monitoring is via anti-factor $\mathrm{Xa}$ (antiXa) blood levels. There are limited data on enoxaparin dosing and monitoring in pediatric cardiac patients. Our study objective was to determine the dose required to obtain desired antiXa levels with standard enoxaparin therapy in pediatric cardiac disease. Methods: Retrospective chart review of cardiology and cardiac surgery patients $<18$ years of age who received at least two enoxaparin doses between 2002 and 2006. Patients with mechanical heart valves or renal dysfunction were excluded. Data collection included: age, weight, underlying diagnosis, BUN/Serum creatinine, type of surgery, initial/final enoxaparin dose, dose changes, antiXa levels, time of dose and antiXa level, and any documented bleeding episodes. Effective anticoagulation was defined as antiXa levels of $0.1-0.5 \mathrm{U} / \mathrm{ml}$ for prophylaxis and $0.4-1 \mathrm{U} / \mathrm{ml}$ for treatment. The patients were grouped according to age: $<2$ months; 2-12 months; 12 months -18 years, and therapy courses were divided by indication and initial dose: prophylaxis ( $<2$ months: $0.75 \mathrm{mg} / \mathrm{kg}$ Q12 hours; $>2$ months: $0.5 \mathrm{mg} / \mathrm{kg}$ Q12 hours) and treatment ( $<2$ months: $1.5 \mathrm{mg} / \mathrm{kg}$ Q12 hours; $>2$ months: $1 \mathrm{mg} / \mathrm{kg}$ Q12 hours). Results: A total of 59 courses of enoxaparin were evaluated with exclusion of 18 courses. The remaining 41 courses (prophylaxis $=5$; treatment $=36$ ) were included. Of 94 antiXa levels obtained, only $61.7 \%$ were appropriately timed (4-6 hours following dose). 
No patients experienced documented bleeding episodes. All 5 prophylaxis patients were $>2$ months old with all initial and final antiXa levels in therapeutic range, and no patients had a dose change. The final mean dose was $0.56 \mathrm{mg} / \mathrm{kg}$ Q12 hours. Thirty-six patients received treatment dosing with the final mean dose as follows: $<2$ months old: $1.54 \mathrm{mg} / \mathrm{kg} ; 2-<12$ months: $1.52 \mathrm{mg} / \mathrm{kg} ; 1-<18$ years: $0.94 \mathrm{mg} / \mathrm{kg}$. Treatment levels in therapeutic range increased from $54 \%$ at initial dose to $75 \%$ at final dose. Thirty-six percent of patients required dose changes based on antiXa levels during therapy. Conclusions: Therapeutic enoxaparin dosing is often suboptimal based on age in pediatric cardiac disease. To achieve therapeutic treatment antiXa levels, the following dosing guidelines should be followed: $<1$ year: $1.5 \mathrm{mg} / \mathrm{kg}$ Q12 hours; $>1$ year: $1 \mathrm{mg} / \mathrm{kg}$ Q12 hours. To achieve appropriate prophylaxis, dosing should be initiated as follows: $<2$ months: $0.75 \mathrm{mg} / \mathrm{kg}$ Q12 hours; $>2$ months: $0.5 \mathrm{mg} / \mathrm{kg}$ Q12 hours. The biggest challenge to antiXa level interpretation is appropriate level timing. Despite challenges, enoxaparin therapy in pediatric cardiac disease should be routinely monitored with antiXa levels appropriately timed at 4-6 hours following dose.

Correspondence to Cynthia Barclay: cynthia.barclay@cchmc.org

The hemodynamic effects of Levosimendan and Milrinone in neonate and older hearts

Signe Holm Larsen, Janus Adler Hyldebrandt, Michael Rahbek Smidt, Vibeke Hjortdal, Hanne Ravn

\section{Aarbus University Hospital - Skejby, Denmark}

Background: Levosimendan (Simdax ${ }^{\circledR}$, Abbott Scandinavia AB, Solna, Sweden) and Milrinone (Corotrops ${ }^{\circledR}$, Sanofi Synthlabo, Bromma, Sweden) are both inodilators used in the treatment of decompensated heart failure following cardiac surgery. In congenital heart surgery the postoperative care for neonates has largely been based on studies in adults. Since myocardial metabolism and receptor kinetics in newborn and older hearts are very different, we aimed to compare the hemodynamic effects of these drugs in newborn piglets and piglets aged four to six weeks. Methods: Twenty-four landrace newborn piglets, all younger than 10 days (mean weight $2.2 \mathrm{~kg}, \mathrm{SD} 0.4$ ) and 25 landrace older piglets, age four to six weeks (mean weight $18.9 \mathrm{~kg}$, SD1.9) were randomised to receive either Levosimendan $(\mathrm{n}=8$ for the group of older piglets, $\mathrm{n}=9$ for newborn piglets), Milrinone ( $\mathrm{n}=8$ for both groups) or saline as controls ( $\mathrm{n}=8$ for both groups). For Levosimendan a bolus of $12 \mu \mathrm{g} / \mathrm{kg}$ was administrated, followed by an infusion of $0.1 \mu \mathrm{g} / \mathrm{kg} / \mathrm{min}$. After 60 minutes a bolus of $24 \mu \mathrm{g} / \mathrm{kg}$ was followed by infusion of $0.2 \mu \mathrm{g} / \mathrm{kg} / \mathrm{min}$. For Milrinone a bolus of $50 \mu \mathrm{g} / \mathrm{kg}$ was administrated, followed by infusion of $0.3 \mu \mathrm{g} / \mathrm{kg} / \mathrm{min}$. After 60 minutes a bolus of $75 \mu \mathrm{g} / \mathrm{kg}$ was followed by infusion of $0.6 \mu \mathrm{g} / \mathrm{kg} /$ min. Cardiac output (CO), systemic and pulmonary artery pressures were measured after 120 minutes. Data analysis was performed with multiple linear regression. Data are presented as relative changes compared to placebo with $95 \%$ confidence intervals. Results: In the group of older piglets $\mathrm{CO}$ increased for both Levosimendan $(27 \%, 2.6-57.7 \% ; \mathrm{p}=$ 0.030 ) and Milrinone (23\%, 0.0-53.1\%; p $=0.054)$. Surprisingly neither Levosimendan nor Milrinone caused any change in $\mathrm{CO}$ in the group of newborn piglets. When looking at mean arterial pressure (MAP) there was no difference between the three treatments in the group of older piglets. However, in the group of newborn piglets there was a significant decrease in MAP for both Levosimendan $(32.4 \%$, 9.5-49.5\%; $\mathrm{p}=0.011)$ and Milrinone $(35.5 \%$, $12.9-52.2 \% ; \mathrm{p}=0.006)$. When adjusting for MAP in the analysis of $\mathrm{CO}$ there was still a tendency towards increase in the group of older piglets for Levosimendan $(25.8 \%,-0.01-59.1 \% ; \mathrm{p}=0.055)$ and Milrinone $(23.2 \%,-4.1-58.3 \% ; \mathrm{p}=0.097)$, but no difference in $\mathrm{CO}$ for the group of newborn piglets. Looking at mean pulmonary artery pressure there was no difference between treatment groups for both groups of piglets. Furthermore there was no difference in pulmonary artery pressure over time between the two groups of piglets. Conclusions: Our data suggests that there seems to be an age-dependent difference in hemodynamic response with the use of both Levosimendan and Milrinone. In a group of piglets aged four to six weeks, we observed an increase in $\mathrm{CO}$ without a decrease in MAP or mean pulmonary pressure. However, in a group of newborn piglets $\mathrm{CO}$ was not increased, instead a marked decrease in MAP was observed. Further studies are needed to explore the patophysiology behind this difference.

Correspondence to Larsen Holm Signe: signe_holm_larsen@ hotmail.com

\section{Tracheostomy following pediatric cardiac surgery: frequency, indications and outcomes}

Timothy Cotts, Jennifer Hirsch, Robert Gajarski

University of Michigan, C.S. Mott Children's Hospital, Ann Arbor, MI, USA 
Background: The prevalence of and indications for tracheostomy in pediatric post-cardiotomy patients is unknown. This study was designed to review baseline characteristics and outcomes of infants requiring tracheostomy following cardiac surgery. Methods: A single center, retrospective review of patients requiring tracheostomy following cardiac surgery between 1/1999-12/2005 was performed. Recorded baseline characteristics included age and weight at time of surgery, primary cardiac diagnosis, major comorbidities, and surgical procedure. The indications for tracheostomy were documented as well as hospital survival, intermediate-term survival, and successful completion of staged single ventricle palliation. Results: Post-cardiac surgery, 59 patients required tracheostomy with a median age of 15 days, and weight of $3.5 \mathrm{~kg}$. Biventricular repair was performed in 33 patients and univentricular repair in 26. Common diagnoses for the biventricular group included conotruncal defects (39\%), coarctation 1 VSD $(21 \%)$, and vascular ring $(12 \%)$. The single ventricle group included HLHS (62\%), unbalanced AVSD (23\%), other complex single ventricle $(11 \%)$ and tricuspid atresia (4\%). Additional major congenital anomalies or presence of a genetic syndrome occurred in $60 \%$ of biventricular patients vs. $15 \%$ of single ventricle patients $(\mathrm{p}<.05)$. For all patients, the indications for tracheostomy included: non-specific failure to wean from mechanical ventilation in $29 \%$, tracheomalacia in $20 \%$, hemidiaphragm paralysis in $17 \%$, vocal cord paralysis in $17 \%$, and isolated upper airway issues in $12 \%$. Overall hospital survival was $75 \%$ with no difference in survival between the biand univentricular groups ( $73 \%$ vs. $81 \%, \mathrm{p}=\mathrm{NS}$ ). Intermediate-term (median 25.5 months, range 1.5-89) follow-up data was available for $54(91 \%)$ patients. Overall survival was $52 \%$, with $60 \%$ and $42 \%$ survival in the bi- and univentricular groups, respectively $(\mathrm{p}=\mathrm{NS})$. There was more late deaths in the single ventricle group occurring in $45 \%$ vs. $12 \%$ in the biventricular repair group $(\mathrm{p}<.05)$. Of the initial 26 single ventricle patients, 5 (19\%) successfully completed the Fontan procedure. No patients who were ventilator dependent at time of discharge following their tracheostomy successfully completed the Fontan palliation. Patients who were not ventilator dependent at time of discharge following tracheostomy had greater likelihood of survival $(67 \%$ vs. $20 \%, \mathrm{p}<.05)$ and a greater rate of Fontan completion than did those requiring chronic mechanical ventilation $(38 \%$ vs. $0 \%$, $\mathrm{p}<0.05)$. Conclusions: Ventilatory failure and tracheomalacia comprise nearly $50 \%$ of the indications for tracheostomy in pediatric patients following cardiac surgery which is associated with significant early and late mortality. Single ventricle patients have the highest late death rate and those with chronic ventilator dependency were unlikely to undergo successful Fontan completion.

Correspondence to Timothy Cotts: cottstim@umich.edu

\section{Impact of dexmedetomidine on opioid and benzodiazepine dosing requirements in children after congenital heart surgery}

Amy Helvie, Chad K. Knoderer, Iftekhar D. Kalsekar, Brandon T. Kibby, Michael S. Mazurek

\section{Clarian Health, Riley Hospital for Children, Indianapolis, IN, USA}

Background: Opioids and benzodiazepines are the cornerstone of pain and sedation management in critically ill children. Dexmedetomidine is a potent and selective alpha ${ }^{2}$-adrenergic agonist with sedative, analgesic, and anxiolytic effects. In adults, dexmedetomidine has been shown to decrease dosing requirements of opioids. Limited data have been published regarding dexmedetomidine use in children. It may be hypothesized that similar to adult data, the addition of dexmedetomidine to standard pain and sedation management protocols in pediatric patients may reduce overall narcotic and benzodiazepine dosing requirements. The primary objective of this retrospective study was to determine the difference in narcotic and benzodiazepine dosing requirements in children treated with and without dexmedetomidine after cardiac surgery. Methods: This retrospective cohort study was conducted after approval from the institutional human subjects review board. A computer-generated list of pediatric cardiovascular surgery patients less than 18 years was used to capture both case and comparison subjects. The case group consists of those patients who received dexmedetomidine infusions initiated within 24 hours after surgery, and were compared to a historical comparison group of pediatric patients who did not receive dexmedetomidine post-operatively. Baseline demographic and clinical characteristics, including Risk Adjusted classification for Congenital Heart Surgery, of the patients in the two groups were compared. Dexmedetomidine, opioid, and benzodiazepine dosing requirements were collected via medication administration records. Opioid dosing was standardized by using equipotent morphine doses. Dosing requirements between the two groups were compared 
to determine the impact of dexmedetomidine on cumulative opioid and benzodiazepine doses. Results: Thirty-six patients in each group were included in the study. No differences in baseline demographics were observed. Dexmedetomidine was continued for an average of $2.4 \pm 1.9$ days with a mean maximum infusion of $0.44 \pm$ $0.17 \mathrm{mcg} / \mathrm{kg} / \mathrm{hr}$. Total daily opioid dosing requirements were reduced $37 \%$ in the dexmedetomidine versus comparison group (morphine equivalents: 2.9 vs. $4.6 \mathrm{mg} / \mathrm{kg} /$ day, respectively, $\mathrm{p}=0.44$ ). Fentanyl infusions were administered to $18(50 \%)$ and $14(38 \%)$ patients in the dexmedetomidine and comparison groups $(\mathrm{p}=0.343)$, respectively. Although not statistically significant, patients in the dexmedetomidine group required fewer days of continuous intravenous fentanyl infusions (mean \pm SD: $4.8 \pm 5.4$ vs. $8.9 \pm 6.7, \mathrm{p}=0.064$ ). The mean and maximum fentanyl infusion doses and mean daily rescue opioid doses did not differ between the two groups. Mean cumulative and daily rescue midazolam dosing requirements were higher in the dexmedetomidine group, although this did not reach statistical significance. Dexmedetomidine was well tolerated in our population. No other adverse effects attributable to dexmedetomidine were observed. Conclusions: Dexmedetomidine may be a useful therapeutic option for pain control in children after surgery for congenital heart disease and may be associated with opioid-sparing benefits. Optimization of dexmedetomidine dosing may provide additional sedative effects with the potential of decreasing benzodiazepine dosing requirements. These data provide additional support for further prospective analyses to determine the impact of dexmedetomidine on analgesia and sedation regimens in critically ill children.

Correspondence to Amy Helvie: ahelvie@clarian.org

Open versus closed methods for endotracheal tube suctioning in postoperative pediatric cardiac patients

Dawn Tucker Heart Institute, Children's Hospital of Orange County,
Orange County, CA, USA

Background: Mechanical ventilation is often required for patients in the Pediatric Cardiovascular Intensive Care Unit. In order to maintain a patent airway, endotracheal tube (ETT) suctioning is frequently performed. Although necessary, ETT suctioning does involve risk. There are two types of endotracheal suctioning techniques utilized: the single, open suction system (OSS) and the closed multi-use suction system (CSS). Because of the nature of their altered cardiovascular status due to cardiac defects and resulting pathophysiology, these children are particularity sensitive to changes in lung volumes and positive end expiratory pressure (PEEP) during ETT open suctioning. Adverse cardiopulmonary changes have been observed during endotracheal suctioning. The removal from positive pressure ventilation during open suctioning alters intrathoracic pressures thereby affecting components of cardiac output. Currently, there is inconsistency regarding suctioning techniques in ventilated patients in the cardiac intensive care units. No technique has emerged as the best practice. The purpose of this study is to evaluate and compare the incidence of ventilator associated pneumonia (VAP) and hemodynamic changes in post-operative cardiac patients having endotracheal suctioning by an OSS vs. CSS. Methods: Using a prospective design sixty-three post-operative open heart patients were enrolled. Patients were randomized to one of the two groups to receive either OSS or CSS. Cardiorespiratory data were recorded before suctioning (baseline), during, one, two, and three minutes after suctioning. Seven cardiopulmonary variables were measured heart rate, $\mathrm{ETCO}_{2}$, blood pressure, central venous pressure, oxygen saturations, and NIRS. VAP were diagnosed according to previously established criteria. Results: Significant changes were seen with $\mathrm{SPO}_{2}, \mathrm{CVP}, \mathrm{ETCO}_{2}$, and NIRS. Both time effects and time by open vs. closed group interactions were statistically significant. Time effect indicates that these variables were changing over the five time periods and furthermore the rate of change was different across the two groups of OSS vs. CSS. During the OSS the oxygen saturations fall and conversely in the CSS the saturations increase $(p<.02)$. Central venous pressure during OSS increased disproportionately to the CSS $(p=.001) . \mathrm{ETCO}_{2}$ slightly decreased during the CSS however returned to baseline rapidly therefore time effect was not statistically significant for CSS. $\mathrm{ETCO}_{2}$ increased during the OSS and remained increased from baseline during the recovery period $(p=.002)$. Both OSS and CSS groups had a decrease in NIRS however the rate of recovery was different between the two groups. The CSS group had a full recovery by the fifth time period and the majority of the OSS group did not recover to baseline $(\mathrm{p}=.049)$. Incidence of VAP No statistical significant difference was found between the two groups. None of the subjects developed VAP. Conclusions: Utilizing the closed suctioning 
system in post-operative pediatric cardiac patients demonstrates less variation in cardiopulmonary status during suctioning. OSS appears to cause changes in intrathoracic pressure altering components of cardiac output. The magnitude of these changes depends partly on factors that influence the underlying cardiovascular status (circulating blood volume, increased pulmonary vascular resistance, ventricular dysfunction) and on factors within the lung. Clearly, endotracheal suctioning of these patients requires thoughtful consideration.

Correspondence to Dawn Tucker: dtucker@choc.org

\section{Safety of intravenous ketorolac use in infants following cardiothoracic surgery}

Cynthia Barclay, Tamara Dawkins, Rhonda Gardiner, Catherine Dent

Cincinnati Children's Hospital Medical Center, Cincinnati, OH, USA

Background: The use of intravenous ketorolac in infants $<6$ months of age is increasing in postoperative cardiothoracic patients. However, there is a paucity of data related to the use of intravenous ketorolac in this age group. This study was designed to evaluate the incidence of side effects of intravenous ketorolac in a study group versus a control group of infants $<6$ months of age undergoing cardiothoracic surgery. The primary endpoint of the study was to measure the frequency of renal impairment and hematologic complications in patients $<6$ months of age who received ketorolac following cardiothoracic surgery compared with those patients who did not receive ketorolac. Methods: A retrospective chart review was conducted on patients admitted to the Cardiac Intensive Care Unit and Cardiac Stepdown Unit who underwent cardiothoracic surgery between January 1, 2004 and April 15, 2007. Patients undergoing single ventricle staged palliation, and those with an allergy to ketorolac or aspirin, and/or abnormal renal and hematologic baseline values were excluded. Data collected included patient demographics, total ketorolac exposure, medication use throughout hospitalization, blood product administration, incidence of renal impairment (increase in BUN and/or SCr two times baseline value) and incidence of hematologic complications $(\mathrm{Hgb}<10 \mathrm{~g} / \mathrm{dL} ; \mathrm{Hct}<29 \%$; or the need for blood transfusion). Results: A total of 61 patients (35 non-ketorolac group and 26 ketorolac group)
$<6$ months of age were evaluated. All patients in the ketorolac group received at least one dose of ketorolac following cardiothoracic surgery. Thirteen patients in the non-ketorolac group were $<1$ week old. The mean dose of ketorolac was $0.5 \mathrm{mg} / \mathrm{kg} / \mathrm{dose}$ IV every 6 to 8 hours. The mean length of therapy was approximately 2 days. The patients receiving ketorolac had no statistically significant change in pre-operative versus post-treatment hematologic or renal status compared to the non-ketorolac group. There was no difference between groups in number of post-operative blood transfusions received. No statistically significant difference was detected between the two groups with respect to amount of narcotic and non-narcotic analgesic use. Conclusions: Ketorolac appears to be safe when used in infants $<6$ months of age with double ventricle anatomy following cardiothoracic surgery. Ketorolac does not appear to significantly increase the risk of hematologic or renal complications compared to patients who did not receive ketorolac post-operatively. Ketorolac does not appear to decrease the use of standard analgesic therapy in infants $<6$ months of age following cardiothoracic surgery.

Correspondence to Cynthia Barclay: cynthia.barclay@cchmc.org

\section{Surgical ligation of patent arterial duct: analysis of 2715 neonates}

Michael Black, S. Jill Ley, Nancy Chorne, Terri Slagle, Richard E. Shaw, Sean O'Brien, Jeffrey E. Jacobs

California Pacific Medical Center, San Francisco, CA, and The Society of Thoracic Surgeons, USA

Background: Increased survival of very-low-birthweight infants (VLBW, defined as those $<1500 \mathrm{~g}$ ) will presumably place increased demand on surgeons for closure of a patent arterial duct (PDA), while altering the risk profile for the population as a whole. Since its first report in 2002, PDA ligation has been the most common neonatal procedure reported to the Society of Thoracic Surgeons (STS) Congenital Cardiac Surgery Database, but neonates $<2500 \mathrm{~g}$ have been excluded from mortality analysis. We sought a review of this data to evaluate the impact of a changing neonatal population on surgical mortality after PDA ligation. Methods: The STS Congenital database was queried regarding the demographics and outcomes of all surgical PDA ligations performed in neonates (age $<30$ days) during a 5-year period from 2002-2006, with 2715 
neonates included in the final sample. Patients were further subdivided into four quartiles by patient weight (<750 g, 750-999 g, 1000-1250, and $>1250 \mathrm{~g}$ ), to better evaluate the impact of patient size on clinical outcomes. Associations between study variables were assessed using stratumadjusted rank correlation chi-square statistics with stratification by hospital identity. Results: Patient age at time of procedure ranged from day of life to 30 days, with most $(32.7 \%)$, occurring between $7-13$ days of age. The overall operative mortality rate was $8.9 \%$, but there was substantial variability by year and patient size (see table below). While changing trends in mortality and patient weight over the 5-year study were not significant, it is noteworthy that VLBW infants comprised $53.1 \%$ of all PDA ligations in 2006. The relationship between mortality and patient weight (by quartile) revealed that patients at the lowest quartile $(<750 \mathrm{~g})$ had significantly higher mortality $(15.9 \%)$, than the other quartiles $(8.6 \%, 5.4 \%$, and $5.1 \%)$, respectively $(\mathrm{p}<0.001)$. Patient age showed a similar trend: mortality at age $<7$ days was $16.6 \%$, compared to $7.7 \%, 7.8 \%$, and $5.1 \%$ when performed during the $2 \mathrm{nd}, 3 \mathrm{rd}$, and 4 th weeks of life, respectively $(\mathrm{p}=0.002)$. Potential limitations of this analysis may include confounding morbidities of prematurity, and possible underreporting associated with a voluntary registry.

\begin{tabular}{llrl}
\hline & $\begin{array}{l}\text { Weight } \\
\text { (median) }\end{array}$ & $\begin{array}{l}\text { VLBW }(<1500 \mathrm{~g}) \\
\text { as \% of PDAs }\end{array}$ & $\begin{array}{l}\text { Operative PDA } \\
\text { Mortality }(\%)\end{array}$ \\
\hline 2002 & 980 & $82 / 184(44.6 \%)$ & $17 / 184(9.24 \%)$ \\
2003 & 970 & $138 / 293(47.1 \%)$ & $19 / 293(6.48 \%)$ \\
2004 & 900 & $290 / 563(51.5 \%)$ & $41 / 563(7.28 \%)$ \\
2005 & 880 & $453 / 833(54.4 \%)$ & $78 / 833(9.36 \%)$ \\
2006 & 890 & $447 / 842(53.1 \%)$ & $86 / 842(10.21 \%)$ \\
\hline
\end{tabular}

Conclusions: As technology has enabled increasing numbers of VLBW infants to survive, management of these fragile neonates bears review. While it is not surprising that operating on such extremely premature patients results in increased mortality trends, a better understanding of these relationships is needed. Are neonatal age and weight interchangeable risk factors? What are the current risks and benefits of surgery versus medical management? Analyses such as these are the first step toward understanding how best to improve care for today's challenging neonate.

Correspondence to Michael Black: blackm@sutterhealth.org
A scientific framework for performance improvement: using Six Sigma methodology to reduce practice variation in the pediatric critical care unit

Christine Agee, Kshitij Mistry, Kristi Ryan, Rebecca Ellis, Judy Oehler, Lisa Cooper, Jon Meliones

Duke University Medical Center, Durbam, NC, USA

Background: The National Association of Children's Hospitals and Related Institutions recommend central venous line (CVL) bundles in order to prevent catheter associated blood stream infections (CA-BSI). Compliance had been unacceptable nine months after implementing the maintenance bundle, leading us to reevaluate our current processes. A data-driven quality improvement initiative (Six Sigma) was instituted. Six Sigma is a rigorous Performance Improvement (PI) platform that utilizes the scientific method to focus on specific processes with high variability and measurability that impact performance. Objective: To identify and improve factors leading to non-compliance with the maintenance bundle components that are critical to quality using Six Sigma DMAIC methodology. Methods: DEFINE: Previously, compliance with maintenance bundle components was measured through nursing self report surveys. Baseline compliance for 3 of the 4 bundle components-line assessment, catheter site care, and tubing care was less than-70\%, 0\%, and 0\%; respectively. A high level process map was developed to define potential key inputs that are critical to quality. MEASURE: A measurement system analysis (MSA) was conducted on the previous measurement system. The MSA demonstrated unacceptably high variability, specifically associated with operator repeatability. ANALYZE: A cause/effect analysis identified all potential critical inputs affecting compliance. The three maintenance bundle components (line assessment, catheter site care, and tubing) were analyzed using a Pareto chart to identify the significant contributing variables critical to compliance quality. The potential root causes within each variable were targeted for improvement. IMPROVE: First, measurement system variability was reduced through operator training and standardization. Next, improvements for each component of the maintenance bundle were individualized. These standards included: 1. Line assessment: implemented line discussion into the child's daily care plan; guaranteeing entire team accountability. 2. Catheter site care: standardized dressing change methods/policy; standard dressing kits; developed CVL handbook for nurses; taught 
dressing changes/aseptic technique on skills day; developed standard care process flow map; individually discussed key CA-BSI prevention tips during competencies. 3. Tubing care: standardized process of how and when tubing/caps should be changed; implemented new labeling system. Results: CONTROL: Following the process improvement initiatives, overall compliance in daily line assessments increased from $65 \%$ to $92 \%$; compliance in catheter site care increased from $0 \%$ to $98 \%$, and compliance in tubing care increased from $0 \%$ to $100 \%$. The maintenance bundle control plan is being instituted to sustain the improvement initiative. Conclusions: The scientific rigor and sophisticated statistical analysis inherent in Six Sigma, provides a valuable framework for PI improvement. Using this methodology we have nearly eliminated variation among standard care procedures such as catheter site care, line assessment, and tubing care. Six Sigma is an enabler in reducing variation of a desired outcome, increasing compliance, focusing attention on factors that are critical to quality, and supporting cultural change. This manufacturing-based approach can be utilized to achieve and sustain success in healthcare; thereby, improving healthcare delivery and ensuring safe patient care.

Correspondence to Christine Agee: christine.agee@duke.edu

Intra-operative hyperglycemia: effect on outcome following congenital heart surgery in infants

William DeCampli, Monica C. Olsen, Hamish Munro, Donald Felix

The Congenital Heart Institute, Arnold Palmer Hospital for Children, Orlando, FL, USA

Background: Both laboratory and clinical studies demonstrate that hyperoxia and cardiopulmonary bypass (CPB) cause intra-operative and post-operative hyperglycemia. In turn, hyperglycemia has been associated, in some studies, with mortality and morbidity following infant cardiac surgery. We studied the characteristics and early outcomes of cases in which hyperglycemia (glucose $>150 \mathrm{mg} /$ dl) was present at the end of CPB. Methods: We reviewed the records of 144 infants $<10 \mathrm{~kg}$ who underwent cardiac surgery with $\mathrm{CPB}$ between $1 / 2006$ and $10 / 2007$. We divided patients into two groups - group A $(\mathrm{n}=72)$ had serum glucose $(\mathrm{GLU})>150 \mathrm{mg} / \mathrm{dl}$ and group $\mathrm{B}(\mathrm{n}=72)$ had GLU $\leqslant 150 \mathrm{mg} / \mathrm{dl}$ at the end of CPB. We further stratified each group by RACHS-1 (Risk Adjust- ment in Congenital Heart Surgery) score - 2 $(\mathrm{n}=33$ in $\mathrm{A}, \mathrm{n}=20$ in $\mathrm{B}), 3(\mathrm{n}=21,22), 4$ $(n=13,21), 6(n=5,9)$. There were no patients in RACHS category 1 or 5 . All patients received pre-CPB dexamethasone $1 \mathrm{mg} / \mathrm{kg}$ and no patient received intra-operative insulin. Patients with GLU $<60 \mathrm{mg} / \mathrm{dl}$ were treated with dextrose. Ultrafiltration during $\mathrm{CPB}$ was used in all patients. After an initial dose of $300 \mathrm{U} / \mathrm{kg}$ heparin, maintenance doses during CPB were given to keep activated clotting time $>400 \mathrm{sec}$. We used t-tests with Bonferroni correction and Fisher exact test (RACHS category 6) to compare groups. Data are expressed as mean and standard deviation. Results: GLU at the end of CPB was $179 \pm 33 \mathrm{mg} / \mathrm{dl}$ in Group $A$ and $128 \pm 27 \mathrm{mg} / \mathrm{dl}$ in Group B (p<.0001). Group A had lower mean RACHS score $(2.8 \pm 1.0$ vs. $3.5 \pm 0.9, \mathrm{p}=.001)$, shorter $\mathrm{CPB}$ time $(142 \pm 63$ vs. $172 \pm 75 \mathrm{~min}, \mathrm{p}=.007)$, greater pre-CPB GLU $(97 \pm 25$ vs. $83 \pm 30 \mathrm{mg} / \mathrm{dl}, \quad \mathrm{p}=.002)$ greater prime GLU (204 \pm 66 vs. $176 \pm 66 \mathrm{mg} / \mathrm{dl}, \mathrm{p}=.01)$ and smaller ultrafiltration volume $(740 \pm 451$ vs. $1001 \pm 658 \mathrm{cc}, \mathrm{p}=.003$ ). First post-operative GLU was significantly higher in group $A$ in RACHS categories $2(\mathrm{p}=.005)$ and $4(\mathrm{p}=.02)$, and serum lactate at 24 hours was higher in group B, RACHS category $4(\mathrm{p}=.05)$. Post-operatively the following quantities were not significantly different between groups A and B for all RACHS categories: Mechanical ventilation time (days) (RACHS 2: $1.5 \pm 2$, $1.7 \pm 2.2$; RACHS 3: $3.4 \pm 4.3,5.1 \pm 6.4$; RACHS $4: 11.5 \pm 17.7,7.0 \pm 4.6$; RACHS 6: $22.7 \pm 16.8$, $20.3 \pm 36.9$ ), length of stay (days) (RACHS 2: $6.6 \pm 3,7.6 \pm 3.4 ;$ RACHS 3: $13.2 \pm 11.8$, $14.1 \pm 11.7$; RACHS 4: $22.4 \pm 24.5,20.5 \pm 13.3$; RACHS 6: $34.8 \pm 25, \quad 56.9 \pm 63.9)$, mortality (RACHS 2: 1,0; RACHS 3: 0,1; RACHS 4: 0,1 ; RACHS 6: 1,1). The incidence of seizures and post-operative infections was also similar in both groups for all RACHS categories. Conclusions: Intraoperative post-CPB hyperglycemia itself does not appear to be a risk factor for post-operative morbidity and mortality in infant cardiac surgery. Elevated values of serum glucose in this study were associated with greater pre-CPB serum glucose and greater glucose concentration in the pump prime solution.

Correspondence to William DeCampli: william.decampli@ orhs.org

Using Six Sigma methodology to improve handoff communication of congenital heart disease patients 
Kshitij Mistry, Jon N. Meliones, James Jaggers, Andrew J. Lodge, Michael Alton, Jane M. Mericle, Karen S. Frush

\section{Duke University Medical Center, Durbam, NC, USA}

Background: During the analyses of adverse events, a commonly cited system breakdown is the sharing of patient information among healthcare providers. Furthermore, the transfer of patient information between healthcare providers - the handoff - has been recognized as a system vulnerability to adverse events. Communication among healthcare providers is particularly important in high-intensity, highstress environments. Children with congenital heart disease (CHD) are at increased risk for adverse events because of clinical complexity and instability, especially following a surgical intervention. A delay in communication of critical information (handoff) may prevent timely intervention and lead to patient destabilization. Methods: The manufacturing industry has long applied quality improvement methodologies to improve performance. Implementing these approaches in healthcare, however, must take into account outcomes important to patients as well as evaluating unintended effects. One performance improvement tool that is increasingly applied to healthcare is Six Sigma. This structured approach utilizes the "DMAIC" process - define the problem, measure the current system, analyze potential sources of variation, improve the process, and control future performance. Improving operational efficiency and maintaining higher process quality results in better patient care. The study took place at a free-standing, tertiary-care children's hospital. A 20-bed pediatric intensive care unit cares for patients of all subspecialties, including those requiring extracorporeal life support and admits approximately 1200 children per year; an estimated $25 \%$ of these patients have undergone surgical intervention for cardiac defects. The study population consisted of anesthesia, surgery, and pediatric ICU providers involved in the hand-off process of children with congenital heart disease following a surgical procedure. Data was collected on 29 patient hand-offs prior to and 142 patient handoffs following performance improvement initiatives; these included: developing prioritization algorithms, training in communication techniques (for example, the "SBAR format"), assigning team roles, and standardizing data set collection. Results: Applying Six Sigma methodology has provided leadership structure, facilitated organizational learning, and has resulted in improved clinical metrics, such as a reduction of turnaround time $(15.3$ to $9.6 \mathrm{~min}$; $\mathrm{p}<0.001)$ and lab draw time $(13.0$ to $2.4 \mathrm{~min}$; $\mathrm{p}<0.001)$; an increase in chest radiographs completed $(60 \%$ vs. $94 \% ; \mathrm{p}<0.01)$ and percent of patients placed on bedside cardio-respiratory monitoring $(86 \%$ vs. $99 \%$; $p<0.01)$ within unit standards. Conclusions: Handoff delays frequently occurred in children undergoing surgical intervention for congenital heart disease. Performance improvement initiatives, such as team training and standardizing the handoff process, provided a systematic approach to solving this complex healthcare delivery issue. Performance improvement initiatives can improve the process of patient handoffs and subsequently decrease delays of time-sensitive therapies. This approach may also be useful in other patient populations and can provide a model for meeting JCAHO National Patient Safety Goals.

Correspondence to Kshitij Mistry: kshitij.mistry@duke.edu

\section{ICU practice patterns for management of adults following congenital heart surgery}

Joylyn Headings, Ross Ungerleider, Karl Welke

Oregon Health E Science University, Portland, OR, USA

Background: Due to the dichotomy of their being adult in age, with cardiac physiology better understood in the pediatric world, adults with congenital heart disease (ACHD) are often treated in systems that are not designed to suit their unique needs. This study was undertaken to describe the unique practice patterns that exist in pediatric (PICU) and adult intensive care units (AICU) for the management of adults following congenital heart surgery (ACHS). Our hypothesis is that, despite treating a comparable group of patients having undergone similar surgical procedures, there is substantial variability in the care of ACHD, depending on whether the patient is in a PICU or AICU. Methods: We identified 142 adult patients ( $\geqslant 18$ years) undergoing surgical repair for a congenital heart lesion between December 2000 and August 2007. For comparison, we also reviewed the management of 75 adolescent patients (12-17 years), with similar defects, operated on during the same time frame. All surgery was performed by the same congenital heart surgery team (surgeons, anesthesiologists, perfusionists, OR nurses, etc). In general, according to the system in our medical center, patients $\geqslant 18$ years were managed in an adult cardiac ICU (AICU), whereas those $<18$ years were managed in a multidisciplinary pediatric ICU (PICU). Results: 217 patients were identified 
$(142 \geqslant 18$ years-adults; $75 \quad 12-17$ years-adolescents). Patients were categorized into 6 major diagnostic categories: 1) Isolated valvular disease $[\mathrm{N}=99$ adults $(67 \%), 41$ adolescents $(51 \%)]$, 2) Valvar disease + Maze procedure $[\mathrm{N}=7$ adults (5\%), 2 adolescents (3\%)], 3) Intracardiac shunt lesions $[\mathrm{N}=21$ adults (14\%), 15 adolescents $(29 \%)]$, 4) Primary arrhythmia $[\mathrm{N}=1$ adult (1\%), 1 adolescent (1\%)], 5) Single ventricle physiology $[\mathrm{N}=3$ adults (2\%), 1 adolescent $(1 \%)]$, and 6) Other $[\mathrm{N}=17$ adults $(11 \%), 20$ adolescents (25\%)]. For patients $\geq 18$ years, $76 \%$ were managed in the AICU; $24 \%$ in the PICU. In the adolescent comparison group (12-17 years), 1\% were managed in the AICU; 99\% in the PICU. Certain practice patterns for these similar patients emerged, and were evaluated between the two ICU systems: 1) Time to extubation; 2) ICU length of stay; 3) Inotrope utilization; 4) blood product utilization. Mean time to extubation for patients admitted to the AICU was 27 (4-452) hours, and for those in the PICU was 21 (3-307) hours. Mean ICU length of stay was 2.3 days (1-17) in the AICU, and 2.9 days (1-36) in the PICU. Conclusions: ACHD patients represent a unique population not cared for ideally by systems that are either designed for adults with acquired heart disease or pediatric patients. Though care providers within each system may have varying levels of expertise and comfort with certain aspects of ACHS care, we found that there were no significant differences in the practice patterns of time to extubation, ICU length of stay, inotrope utilization, and utilization of blood products, for similar groups of patients, between AICU and PICU.

Correspondence to Joylyn Headings: headings@ohsu.edu

\section{Noninvasive positive pressure ventilation in pediatric cardiosurgical patients with postextubation respiratory failure}

Lubica Kovacikova, Dobos Dusan, Skrak Peter, Zahorec Martin

\section{Intensive Care Unit, Pediatric Cardiac Center, Bratislava, Slovakia}

Background: Noninvasive ventilation is a treatment option in chronic and acute respiratory failure. The aim of this study was to review our initial experience with use of noninvasive positive pressure ventilation (NPPV) in pediatric cardiosurgical patients with postextubation respiratory failure.
Methods: The retrospective study included eight children with a median age of 3.5 years (range, 11 months - 14 years) treated with NPPV for postextubation failure after cardiac surgery. Three patients had two-ventricle repair, five patients had a single ventricle palliation. Before starting NPPV, high flow oxygen via nasal cannula was applied to four patients, and continuous positive airway pressure of $10 \mathrm{~cm} \mathrm{H}_{2} \mathrm{O}$ by nasopharyngeal tube was applied to four other patients. Patients to receive NPPV were fitted with an oronasal mask connected to a flow-triggered pressure assistcontrol ventilator (Servo-i, Maquet Inc., Solna, Sweden). The device allowed a pressure support and/or pressure control mode. Hemodynamic and respiratory parameters and blood gases were measured prior to and 1, 3, 6, 12 and 24 hours after institution of NPPV. Need for sedation and complications of NPPV were recorded. Results: NPPV was instituted at median of 142 hours (range, 25-1100 hours) after cardiac surgery and at median of 19 hours (range, 11-216 hours) after extubation. Within the first hour of NPPV, the respiratory and heart rate decreased from median value of 45 breaths $/ \mathrm{min}$ (range, $22-55$ breaths $/ \mathrm{min}$ ) to 34 breaths $/ \mathrm{min}$ (range, $21-45$ breaths $/ \mathrm{min}$ ) $(\mathrm{p}=0.01)$ and from 146 beats $/ \mathrm{min}$ (range, 99172 beats $/ \mathrm{min}$ ) to 134 beats $/ \mathrm{min}$ (range, 99-156 beats $/ \mathrm{min})(\mathrm{p}=0.01)$, respectively. The arterial oxygen tension, arterial oxygen saturation and the oxygenation ratio increased from $6.85 \mathrm{kPa}$ (range, $6.00-8.63 \mathrm{kPa})$ to $8.12 \mathrm{kPa} \quad(6.77-16.0 \mathrm{kPa})$ $(\mathrm{p}=0.01$ ), from 0.83 (range, $0.79-0.96)$ to 0.92 (range, $0.79-0.99)(\mathrm{p}=0.009)$ and from 15.2 (range, 10.0-32.3) to 20.1 (range, 11.2-40.0) $(\mathrm{p}=0.008)$, respectively. Improvement in clinical parameters and blood gases persisted during the first 24 hours. No significant change was observed in systolic or diastolic blood pressure, arterial carbon dioxide tension, $\mathrm{pH}$, or bicarbonate concentration. During the period of NPPV median value of maximum positive end-expiratory pressure setting among patients was $5.5 \mathrm{~cm} \mathrm{H}_{2} \mathrm{O}$ (range, $3-10 \mathrm{~cm} \mathrm{H}_{2} \mathrm{O}$ ). Pressure support was set at $10 \mathrm{~cm}$ $\mathrm{H}_{2} \mathrm{O}$. In 5 patients Pressure Control mode with preset respiratory rate of 20 breaths $/ \mathrm{min}$ (range, $10-26$ breaths/min) and inspiratory pressure of $20 \mathrm{~cm} \mathrm{H} \mathrm{H}_{2} \mathrm{O}$ (range, $14-22 \mathrm{~cm} \mathrm{H}_{2} \mathrm{O}$ ), was used. NPPV avoided tracheal intubation in 6 of 8 patients. In these patients median duration of ventilation was 133 hours (range, 75-1320 hours). One patient with chronic lung disease required intubation following 10 hours of NPPV due to severe bronchospasm and the second patient after 21 hours due to refractory ventricular arrhythmia. NPPV was tolerated well in all patients. In one 
patient skin breakdown over nasal bridge occurred. Conclusions: In pediatric cardiosurgical patients with postextubation failure NPPV improves vital signs and gas exchange and may avoid the need for tracheal intubation. Its use is associated with good tolerance and a low rate of complications. Patients should be monitored closely for signs of possible noninvasive ventilation failure.

Correspondence to Lubica Kovacikova: lkovacikova@yahoo.com

\section{Documentation of pain assessment following paediatric heart surgery: evolving practices}

Carrie Heffernan, Marie Pinard, Connie Chan, Carolyn Kaulback

\section{The Hospital for Sick Children, Toronto, ON, Canada}

Background: Gaps in pain management were identified at SickKids based on the results of a 2005-2006 patient and family satisfaction survey and a hospital-wide pain audit. In response, the Labatt Family Heart Centre (LFHC) has made improving pain management a priority and conducted a documentation audit of post-operative pain assessment on the 29 bed Cardiology inpatient unit in March of 2007. Best Practice Guidelines suggest that the consistent use of a systematic and validated pain assessment tool is essential for effective pain management (RNAO, 2007). Therefore, the focus of the audit was to measure the frequency of pain assessment documentation using a valid pain tool, as well as to highlight possible areas for improvement. Methods: Patients who were $<4$ days post heart surgery or between 5-10 days post-operative with a chest tube in place, are considered to require regular pain assessments ( $\mathrm{q} 4$ hour minimum) and therefore were included in the audit. A data collection tool was created to facilitate consistency of information gathered. It is recognized that pain assessments conducted that were not documented on the patients' bedside flow sheet record were not captured in the results and is a limitation of this audit. Results: The frequency of pain assessment documentation was determined based on the number of opportunities in accordance with the hospital's "Pain Assessment" policy. The data was further stratified by age and whether narcotics were administered. Overall, the results showed that documentation of pain assessments only occurred $21 \%$ of the time when required for this patient group. In particular, neonates $(<2$ mos.) fared the poorest, although they only accounted for $5 \%$ of the sample size. Anecdotal feedback from nursing staff suggest that the currently available pain assessment tool for the neonatal $(<2 \mathrm{mo})$ population, is labour intensive, complex and possibly better suited for procedural pain assessment. It was also noted that the consistent documentation pre and post the administration of narcotics also requires improvement. Conclusions: In response to the audit findings, several recommendations for the LFHC interprofessional team (IPT) were developed and disseminated. These recommendations are as follows: a) incorporate inquiries about pain assessments as a regular part of the inpatient daily IPT rounds, b) explore an alternate valid pain assessment tool suitable for the post-operative neonatal age group $(<2$ mos.), c) teach and encourage patient and families to participate in pain management by using developmentally appropriate valid pain tools throughout hospitalization, and d) monitor ongoing progress by setting target goals for future pain audit results as an IPT incentive. A comprehensive and motivated IPT collaboration (Director, Managers, Cardiologists, Quality Management, Nurses, Educator) was essential to the success of all phases of the audit. Furthermore, the development of successful recommendations was also contingent on an IPT that acknowledged pain improvement strategies as a team responsibility that requires several layers of intervention which are ultimately sustained by a shift in clinical practice culture. At this time, several of the recommendations are currently in progress and the results of future re-audits will be presented.

Correspondence to Carrie Heffernan: carrie.heffernan@sickkids.ca

\section{Utility of the routine chest radiograph \\ following removal of a chest tube in pediatric postoperative cardiac patients}

Betsy Gerrein, Wayne Mays, Martha Willis

\section{Cincinnati Children's Hospital Medical Center,} Cincinnati, OH, USA

Background: Cardiac defects are the most common congenital abnormalities. Many children who undergo corrective surgical procedures will receive one or more chest tubes. This study examined the utility of routine chest radiographs (CXR) in the evaluation of the presence of a pneumothorax (PTX) following the removal of the chest tube in the pediatric postoperative cardiac patient. Methods: A retrospective chart review was conducted for patients from newborn to 18 years of age who 
underwent open heart cardiopulmonary surgery between January 1, 2006 and June 30, 2007 and also had at least one chest tube placed. Charts were reviewed for the presence of PTX at time of CXR. Charts were also reviewed for decreased $\mathrm{O}_{2}$ saturation, dyspnea, retractions, tachypnea, increased $\mathrm{O}_{2}$ requirement and change in breath sounds in patients with and without PTX. These signs and symptoms were used to predict the presence of PTX at time of CXR following chest tube removal. The data were analyzed using logistic regression to determine what variables are related to the likelihood of PTX. The data were analyzed for differences in proportions of signs and symptoms manifested among patients with and without PTX. Contingency tables and frequency distribution were employed to display results. Results: A total of 222 patients met the inclusion criteria (127 males, 95 females). The patient mean age was $3.7 \pm 4.9$ years. PTX following chest tube removal was present in $21(9.5 \%)$ of the patients at CXR. Logistical regression of the signs and symptoms was not predictive of the presence of PTX. Chi square frequency analysis of the presence of decreased $\mathrm{O}_{2}$ saturation, dyspnea, retractions, tachypnea, increased $\mathrm{O}_{2}$ requirement and change in breath sounds was not significant. The presence of PTX was accurately predicted in 2 out of 21 occurrences yielding a $9.5 \%$ true positive and $90.5 \%$ false negative rate. Clinical signs and symptoms were present in patients with and without PTX. Conclusions: PTX at CXR following chest tube removal was present in $9.5 \%$ of this patient sample. This study determined that clinical presentation was not adequate to accurately predict the presence of PTX at CXR. However, no patients required additional therapeutic intervention for the treatment of PTX in this patient sample.

Correspondence to Betsy Gerrein: betsy.gerrein@cchmc.org

\section{Parental participation in clinical rounds in a pediatric cardiac ICU}

Maureen T. Rorke, Karen Bergan, Shevy Kahan, Carolyn Kyne

\section{Morgan Stanley Children's Hospital of New York Presbyterian}

Background: Parental participation in rounds has been noted to increase satisfaction, decrease anxiety, and improve efficiency and outcomes in parents of children in the Cardiac ICU. The objective of this project is to increase parental participation in rounds through a partnership with the Family Advisory Council (FAC). Prior to the project, parents expressed a degree of conflict about whether they were welcomed to listen to discussions during rounds about their child's care, and how to ask questions about what they heard. Some parents preferred not to listen \&/or participate in rounds, but rather speak to a familiar physician or nurse about the plan of care after rounds. A committee was formed including representatives from the FAC, ICU physicians and nurses, to explore ways to offer parents the option of participation in rounds. Methods: Dialog with members of the FAC revealed the desire of family members to be aware of, and understand the daily plan of care, and expected interventions and accomplishments throughout the hospital course. Focus groups held with physicians revealed concerns about the ability to teach residents and medical students, discuss abnormal results, and potentially adverse outcomes with parents present. Several educational sessions were presented by visiting physicians and nurses from institutions where parental participation had been implemented. Nurses designed a questionnaire which was given to the parents of children in the ICU, inviting them to participate in rounds, and giving them the ability to accept or decline. Parents who accepted were prepared for what to expect on rounds by their primary nurse; parent and nurse would review any questions the parent might want to ask on rounds. The process was implemented over a two month period. Data was collected on the number of parents participating as a ratio of total census. This data was shared as an agenda item of the monthly Critical Care Quality Improvement Committee, and one actual case per month was reviewed for benefits and risks. Results: Parental participation in rounds increased as a result of offering parents the option of attending, and support by their primary nurse. Physician resistance to parental participation decreased. Physicians experienced less disruption or change in the way rounds were conducted, than originally anticipated. Parents expressed greater satisfaction and less anxiety, because they not only knew and understood the daily plan of care and hospital course, but could participate by contributing information on their child's response to interventions. Conclusions: Parental participation in rounds can have a positive influence to decrease parental anxiety about their child's care, and increase satisfaction through prompt communication about the daily plan of care and hospital course. Clinical rounds need not be restructured or altered to accommodate parental participation. Further study and measurement of decreased anxiety or increased satisfaction is 
warranted, as well as study of improvement in outcome \&/or length of stay.

Correspondence to Maureen Rorke: mtr0046@msn.com

\section{Extended use of dexmedetomidine in the pediatric cardiothoracic ICU}

Sharon Beijan, Cassie Valasek, John J. Nigro, David Cleveland, Brigham C. Willis

\section{Eller Congenital Heart Center, St. Joseph's Hospital and Medical Center, Phoenix, AZ, USA}

Background: Dexemedetomidine is an $\alpha 2$-adrenergic agonist that causes sleep-like sedation and mild analgesia. It has been approved for sedation in adults for under 24 hours in an ICU setting. Descriptive studies have detailed its use for longer periods of time in adults, as well as short-term use in pediatric patients. Due to its properties of providing sedation without narcosis or respiratory depression, it may be an effective agent for use in the sedation of patients in the pediatric cardiothoracic ICU. We reviewed our experience with the drug to detail its safety and efficacy. Methods: We conducted a retrospective chart review of all patients who received dexmedetomidine since the opening of a new, dedicated pediatric cardiothoracic ICU in our institution. The study was approved by the institutional investigative review board. Patients were identified from pharmacy records of drug administration. Results: We identified 75 patients that received the drug. The median age of recipients was 5 months (range 0.25-156 mo.). The mean duration of drug administration was $48.4 \mathrm{~h}$ $(2.5-177 \mathrm{~h})$. The mean initial starting dose was $0.38 \mathrm{mcg} / \mathrm{kg} / \mathrm{h}(0.2-0.5)$, and the mean maximum dose used was $0.81 \mathrm{mcg} / \mathrm{kg} / \mathrm{h} \quad(0.25-2.0)$. No patients received a bolus of drug upon initiation. The mean duration of drug continuation after extubation was $13.8 \mathrm{~h}$ (0-44). Physiologically, there were no significant changes in mean arterial pressure, heart rate, respiratory rate, or oxygen saturation before, during, or after utilization of the drug. No patients had severe adverse events, including bradycardia or hypotension upon initiation. There were also no detected clinically significant occurrences of ileus, and only one patient was started on hydrocortisone after initiation of dexmedetomidine. Nursing and physician satisfaction with the drug was excellent. Conclusions: In this limited and retrospective review, dexmedetomidine was found to be safe and efficacious. Use of dexemedetomidine as a sedative agent for extended periods of time in children deserves investigation in a prospective, controlled manner, given its excellent characteristics of hemodynamically stable, nonnarcotic induction of sleep-like sedation without respiratory depression.

Correspondence to Brigham C. Willis: brigham.willis@chw.edu

\section{Extubation in the operating room after corrective surgery of tetralogy of Fallot: seven years of experience}

Iva Tolj, José R. Iribarren, Alfredo Sánchez, Julia González, Jose Castejón, Armando Páez, Alberto Figueira, Brígida Aguerrevere, Claudia Scanzoni, Ma. Clara Lander

\section{Proyecto Todo Corazón, Caracas-Venezuela}

Background: The use of mechanical ventilation (MV) is common in the treatment of patients undergoing surgery for congenital heart disease $(\mathrm{SCH})$. The success of weaning depends crucially on the resolution of the problem that led the MV, the capacity of pulmonary gas exchange, muscle strength, indemnity from the air and the appropriate use of opioids and muscle relaxants. We adopted a strategy to prevent and handle MV extubation in the operating room after surgery. We review our experience. Methods: Objective: To check the rate of early extubation (EE) after CCC for complete overhaul of Tetralogy of Fallot (TF). Methods: Retrospective study. Over a period of 7 years, 142 patients were corrective surgery of TF. The variables preoperative, perioperative and postoperative cardiac and repair, were compiled and analyzed statistically. Results: Within the preoperative variables that can be said of all children under $\mathrm{SCH}$, the weight averaged $(14,91 \pm 6 \mathrm{~kg})$, aged between 5 months and 17 years of perioperative variables, the average time extracorporeal circulation (ECC) was $(71.21 \mathrm{~min})$ while for the time clamp aortic was $(48.86 \mathrm{~min})$, the lowest temperature reached was $22^{\circ} \mathrm{C}$, and no cases requiring full cardiac arrest. In the post operative variables we found a rate of early extubation $(\mathrm{n}=135 ; 95.07 \%)$, compared with failed extubation $(\mathrm{n}=7,4.92 \%)$. With a mortality rate $(\mathrm{n}=12$, 8.51\%. Conclusions: Up to $95 \%$ of children under $\mathrm{SCH}$ to complete repair of TOF, could be extubated so successful, only $5 \%$ of the attempts failed extubation. Being our prevalencida similar to that reported by other authors. The extubation after a CCC can be done safely with the proper selection of patients. There are new studies that establish 
criteria or factors associated to predict what type of patient will implement this strategy extubation within surgery or those who will have to undergo more time with ventilatory support, increased incidences of complications, long stays in the Intensive Care Unit and increased mortality.

Correspondence to Iva Tolj: ivatolj@yahoo.com

\section{Obesity in children with congenital heart disease: what does it mean?}

Trenda Ray

\section{Arkansas Children's Hospital, Little Rock, AR, USA}

Background: The prevalence of childhood obesity and overweight is well documented, and has become the focus of health care professionals around the world. Children with congenital heart disease (CHD) are also affected by this epidemic, but the prevalence of obesity and overweight in children with CHD has not been reported in the literature. In addition to the risk factors shared by all children who are obese, children with CHD may be at particular risk for complications due to parentally or medically imposed activity restrictions. Therefore, the purpose of this study was to explore the prevalence of obesity and overweight in children with CHD. This study also explored the prevalence by cardiac diagnostic category, gender, and age of the participants. Methods: After obtaining IRB approval, the researcher conducted a retrospective review of electronic medical record data of 1318 patients seen in the Cardiology Clinic at Arkansas Children's Hospital over a six month period. Of these, 670 were evaluated for congenital heart disease. Those selected for inclusion were between 5 and 18 years of age. The raw BMI data were converted to BMI $\mathrm{z}$ scores for the purpose of analysis. Children with a BMI $z$ score between the 85th and less than 95th percentiles were categorized as overweight while those with BMI z scores greater than or equal to the 95th percentile were categorized as obese. Descriptive statistics were used to describe the sample and describe the prevalence of obesity and overweight in the sample as a whole and by cardiac diagnosis. Finally, Analysis of variance (ANOVA) was used to explore the relationship between BMI $\mathrm{z}$ score and the following variables: cardiac diagnostic category, gender and age. Results: Just over $14 \%$ of children with CHD were overweight, while $20.6 \%$ were considered obese. There was no significant relationship between BMI z score and cardiac diagnostic category. Therefore, children with cardiac diagnoses were no more or no less likely to be overweight or obese than control children. In addition, the prevalence of overweight and obesity were not significantly different for children with complex CHD when compared to those with simple CHD. Further, there was no significant relationship between either gender or age, suggesting that the prevalence of overweight and obesity are similar for males and females and with advancing age. Conclusions: This study offers an opportunity to better understand the prevalence of obesity and overweight in children with CHD. The prevalence of obesity and overweight was comparable to the prevalence of $17.4 \%-18.8 \%$ reported in the 2003-2004 NHANES data for children in the same age range. Therefore, this study provides further evidence that this population of children are no different from the population of healthy children who are affected by obesity. This research provides information for health care professionals who work with this specific population of children regarding their susceptibility to this national trend. Future studies will focus on the exercise participation in these children, as well as the understanding by their parents on the necessity of physical activity in the prevention of overweight

Correspondence to Trenda Ray: raytd@archildrens.org

\section{The data manager - an invisible but essential role in CHD programs}

Andrea Kennedy, Nancy Burnham, Diane Hartman, J. William Gaynor

The Children's Hospital of Philadelphia, Philadelphia, PA, USA

Background: There are an increasing number of requests from a variety of interested parties for data concerning surgical volume, case mix and outcomes. These parties include researchers, hospital administrators, third-party payors as well as national and international multi-institutional databases. Accurate data is essential to provide reliable and precise information. Patient and procedural data is collected from multiple sources and individuals such as surgeons, perfusionists, nurses, fellows, schedulers, office and research staff. The large number of individuals involved in data entry increases the risk of inaccurate and incomplete data. Without careful verification of data completeness and accuracy, the reported results may provide an incomplete picture of clinical activity and outcomes. This review was 
undertaken to evaluate the role of a dedicated cardiac center data manager in establishing a strategic data management plan. Methods: Retrospective review of requested queries and system processes between 2002 and 2006 was performed to identify areas of improvement since hiring a dedicated data manager in 2004. Results: During the review process we analyzed 82 data requests from both internal and external departments and institutions. Data was provided to over 13 outside institutions such as UNOS, Aetna, Child Life Magazine and US News \& World Report. As an example of database problems, data submitted to the Society of Thoracic Surgeons (STS) was missing $57 \%$ of discharge mortality in 2002. Processes have been implemented to ensure data collection is complete and accurate. Training manuals were created and targeted for each area of collection outlining data elements that are required for reporting an accurate picture of the surgical volume within the institution. This process along with monthly monitoring of these fields has shown a decrease of missing data to $0.2 \%$ in 2006. Conclusions: Implementing these monthly processes and reconciliations has ensured accurate reporting and billing that have allowed continued referrals and reimbursements to the hospital, continued participation as a center of excellence and provided the Cardiac Center with recognition as a leader in the treatment and care of children with congenital heart disease. Internal requests have helped produce grant funding through the University Foundation and successful acceptance of published manuscripts. The Cardiac Center's measured outcomes are dependent upon accurate and reliable responses to data requests which require skilled data management that incorporates knowledge of the centers data structure and communication across multiple disciplines that include Cardiac Anesthesia, Cardiac Nursing, Cardiology, and Cardiothoracic Surgery. Designating a cardiac center data manager as a central resource to all data requests will improve the reliability and accuracy of usable outcomes such as: quality assurance/benchmarking, grant funding, public relations, and scientific publications.

Correspondence to Andrea Kennedy: kennedya@email.chop.edu

\section{Sotalol as first line therapy for fetal supraventricular tachyarrythmias}

Amee Shah, Anita Moon Grady, Kathryn Collins, Theresa Tacy, Lisa K. Hornberger

University of California San Francisco, San Francisco, CA, USA
Background: Untreated fetal supraventricular tachycardia (SVT) and atrial flutter (AF) can be associated with significant morbidity and mortality. Digoxin is typically used as first line therapy, but may require several days to achieve success and is often ineffective in fetal hydrops. Other antiarrhythmics are frequently added to the regimen only after digoxin has failed. In an attempt to achieve more rapid control of SVT and AF in fetuses with or at risk for fetal hydrops, we began using sotalol alone or in combination with digoxin as first line therapy starting in July 2004. This study reviews our experience. Methods: We retrospectively reviewed the medical records of 25 consecutive pregnancies with fetal tachycardia managed in our combined center, assessing treatment and clinical outcomes. Fetal echocardiograms and infant ECGs and Holters were reviewed to determine different characteristics of the tachyarrhythmias. Results: Of 25 pregnancies, 18 (15 with SVT and 3 with AF) were treated with sotalol (80-320 mg PO BID or TID). Twelve of the 18 also received digoxin, including 8 with hydrops at diagnosis. Gestational age at treatment ranged from 17-35 weeks (median age 25 weeks). One fetus developed blocked atrial extrasystoles after 1 dose of sotalol and was delivered for fetal bradycardia. Sotalol was effective postnatal therapy for this infant. Of the 17 others, 5 with SVT and 3 with AF had resolution within 5 days of treatment onset (median 1 day). Five others with SVT showed some response (less frequent episodes, rate slowing and resolution of hydrops) without complete conversion. In 1 with some initial response but persistent ascites, and 2 without response to sotalol and digoxin, additional medications (amiodarone and/or flecainide) were given with subsequent conversion. Three grossly hydropic fetuses with SVT showed no response and died within 1-3 days of treatment. In 1 other with a slow response, the mother chose pregnancy termination. Mechanisms of tachycardia in the 18 included AF (3), and short VA (2), long VA (6), equal AV/VA (4) and indeterminate (3) SVT. All AF patients were in the rapid response group, whereas other mechanisms were represented nearly equally among responders, partial responders, and non-responders. After birth, of the 8 with initial rapid response, only 1 had SVT. Of 5 survivors with more difficult to treat fetal SVT (incomplete response after 5 days of therapy), all have been difficult to treat after birth, including 1 with short VA, 1 long VA, 1 with indeterminate $\mathrm{AV} / \mathrm{VA}$ intervals, and 2 with equal $\mathrm{AV} / \mathrm{VA}$ intervals (one requiring radiofrequency ablation at 3 weeks). Conclusions: Sotalol is an effective antiarrhythmic for treatment of fetal SVT and $\mathrm{AF}$ with a $76 \%$ complete or partial response 
rate in our series. SVTs more difficult to control prenatally continue to be difficult to manage after birth. Long VA SVTs are likely to be a more common mechanism of fetal SVT than previously believed.

Correspondence to Amee Shah: shaha04@gmail.com

Exercise-induced abnormal pulmonary arterial pressure response in adolescents and adults with atrial or ventricular septal defect

Thomas Möller, ${ }^{1}$ Eirik Pettersen, ${ }^{2}$ Kari Peersen, ${ }^{3}$ Per Morten Fredriksen, ${ }^{4}$ Henrik Holmstrøm, ${ }^{5}$ Ekkehard Grünig, ${ }^{6}$ Derliz Mereles, ${ }^{7}$ Erik Thaulow ${ }^{5}$

${ }^{1}$ Pediatric Department, Vestfold Hospital, Tonsberg, Norway; ${ }^{2}$ Department of Medicine, University of Oslo and Rikshospitalet, Oslo, Norway; ${ }^{3}$ Department of Cardiology, Vestfold Hospital, Tonsberg, Norway; ${ }^{4}$ Clinic of Rebabilitation, Faculty of Medicine, University of Oslo and Rikshospitalet, Oslo, Norway; ${ }^{5}$ Pediatric Cardiology Unit, Faculty of Medicine, University of Oslo and Rikshospitalet, Oslo, Norway; ${ }^{6}$ Thoraxklinik, University of Heidelberg, Germany; ${ }^{7}$ Department of Cardiology, Angiology and Pulmology, University Hospital, Heidelberg, Germany

Background: The European Heart Survey on Congenital Heart Disease described follow-up data in 1536 adult patients with isolated atrial (902) or ventricular (634) septal defect (ASD or VSD). The reported prevalence of pulmonary hypertension was $18 \%$ in both closed ASD and closed VSD which was surprisingly high. However, the study recruited patients from specialised centres with a selection towards difficult cases. Our study aims to examine a representative population-based group of young patients with isolated ASD or VSD. The main focus is pulmonary hypertension or its postulated precursor, abnormal pulmonary pressure response (AR) during exercise. Methods: The study comprises 50 patients with either isolated VSD or ASD, whose defects were assumed hemodynamically insignificant or surgically closed early in life. The patients were recruited among all patients born between 1982 and 1993 from two regions in Norway. Exclusion criteria were complex cardiac lesions, neurological or other conditions interfering with exercise testing or compliance and fixed or dynamic right ventricular outflow tract obstruction.

The patients are age- and gender matched against 86 healthy controls. Cardiovascular exercise testing (treadmill protocol) and echocardiography at rest and during supine cycling (target heart rate $160^{-\mathrm{min}}$ ) are performed. Right ventricular performance at rest is measured by tricuspid annular plane systolic excursion (TAPSE). Right atrial pressure is estimated by inspiratory collapse of the vena cava inferior. During exercise systolic pulmonary arterial pressure (PAP) is monitored by measurement of tricuspid valve regurgitation jet velocity. Results: Treadmill testing showed normal (Gaussian) distribution of age-corrected maximal oxygen uptake $\left(\mathrm{VO}_{2}\right.$ peak) for both groups expressed by Z-score of $\mathrm{VO}_{2}$ peak $\left[\mathrm{ml} \times \mathrm{kg}^{-0.67} \times \mathrm{min}^{-1}\right]$ related to a Norwegian normal standard. Highly endurancetrained volunteers with a Z-score $>2$ were excluded. There was a significant $(\mathrm{p}<0.001)$ lower mean Z-score of $\mathrm{VO}_{2}$ peak in the patient group $(-1.64$, range -5.2 to 1.3$)$ as compared to the normally trained controls (mean -0.12 , range -4.5 to 2.0).

In patients with surgically closed defects echocardiography at rest showed a significant lower right ventricular performance measured by TAPSE $(\mathrm{p}<0.001$, means operated patients/control 15.7/ $23.0[-8.6,-6.0])$.

There were no cases of pulmonary hypertension at rest in the patient group.

Exercise echocardiography rendered assessable registrations of tricuspid regurgitation jet velocity in $46 / 50$ patients $(92 \%)$ and in $83 / 86$ controls (97\%).

The maximal PAP during exercise was significantly higher in patients compared to controls $(\mathrm{p}<0.001$, means patients/controls $39 / 32 \mathrm{mmHg}$ $[3.5,10.3])$.

21 of 46 patients $(46 \%)$ and 16 of 83 controls (19\%) had PAP above $40 \mathrm{mmHg}$ during exercise. Eight patients (17\%) but only one control $(1 \%)$ had PAP above $50 \mathrm{mmHg}$ during exercise. The PAP did not differ between genders and no correlation was found between TAPSE at rest and PAP during exercise. Conclusions: Young patients with small untreated or surgically closed isolated ASD or VSD have lower exercise capacity. Patients with surgically closed defects have lower right ventricular performance than healthy individuals. About one out of five patients has pathologic pulmonary pressure load above $50 \mathrm{mmHg}$ during exercise. The long-term implications of these findings have to be investigated.

Correspondence to Thomas Möller: thomas.moller@siv.no

Despite high satisfaction with training, are we missing the opportunity to create experts?

Kelly Nicklas, Coleen Miller, Bronwyn Bartle, Cherylanne Wilson, Jon Meliones 
Duke University Medical Center, Pediatric Cardiac Intensive Care, Durham, NC, USA

Background: The care of children with congenital heart disease is becoming increasingly complex requiring specialized training for all health care providers involved in a cardiothoracic program. In particular, many centers are opting for a specialized cardiothoracic intensive care unit with highly trained nurses. This provides an opportunity to develop advanced nursing training programs and an environment of excellence. Our hypothesis was that an advanced cardiac education program would improve nurses' confidence caring for pediatric cardiovascular patients and create a culture of expertise. Methods: A two-day multidisciplinary advanced cardiac educational program was developed consisting of traditional didactic sessions presented by senior nursing staff. Novel interactive hands on sessions consisted of a porcine heart lab, cardiopulmonary bypass wet lab, and case studies. The session concludes with radiology basics, echocardiography, rhythm identification, and clinical review. Weekly case conferences are conducted to incorporate these principles into daily clinical practice. Pre and post surveys were conducted to evaluate satisfaction and overall sense of expertise. Results: The survey demonstrated that $100 \%$ of nurses attending the advanced cardiac educational program believe they have become a more integral part of the care team, 95\% believe they are practicing safer care, $89 \%$ have increased confidence and can describe abnormal physiology and $84 \%$ have improved satisfaction caring for cardiovascular patients. However, only 9\% of nurses acknowledge themselves as an expert in cardiovascular care. Utilizing Benner's stages of clinical competence as a framework, nurses in our cohort with $<4$ years experience consistently defined themselves as competent or less, whereas nurses with 5-24 years defined themselves as proficient or experts. ${ }^{1}$ All survey participants identified experience as the most valued characteristic of an expert. When the perceptions of clinical competence were further explored three specific groups of respondents emerged: Group 1 (Experts undefined): Redefined themselves as an expert once the definition was modified to; Performing within the top $10 \%$ of the field. Group 2 (Expertise not valued): Believe expertise is too difficult to obtain and not necessary to achieve job satisfaction. Group 3 (Experts in training): Believe expertise is important and strive towards this goal. Conclusions: An advanced cardiac educational program increases the overall confidence of nurses caring for cardiovascular patients. To address the differences within the three newly defined groups, unique strategies need to be adopted. Group 1 can benefit by improved role definition thereby creating the culture of expertise and providing value as well as acknowledgement to this role. Realizing the benefit of creating expertise in Group 1 may motivate group 2. Group 3 benefits from the educational programs described. Recognition of this culture allows leadership to tailor programs to support expertise. We are not alone in our quest, in the AAMC 2007 President's address it is highlighted that institutions must strive to realize and improve culture to reach our goals.

\section{Reference}

1. Benner P. From novice to expert. Am J Nursing 1982; 82: 402-407.

Correspondence to Kelly Nicklas: nick1002@mc.duke.edu

Looking in the coronary sinus: a comparison of epicardial pathways versus right posterior septal pathways in the pediatric population

Edward Rhee, Jennifer Avari

Eller Congenital Heart Center, St. Joseph's Hospital and Medical Center, Phoenix, AZ, USA

Background: Epicardial pathways are defined as pathways that cannot be ablated from an endocardial position and are typically associated with the coronary sinus. While these pathways have been described in adult patients, they have not been systematically described in pediatric patients. We report our experience with epicardial pathway (or coronary sinus accessory pathway, CSAP) ablations in children. Methods: Patients who underwent CSAP or right posteroseptal (RPS) pathway ablation from 11/2004-6/2007 were collected. Retrospective reviews of surface ECG, fluoroscopic and intracardiac electrogram data were then performed to identify electrophysiologic markers of CSAP. Results: Thirteen patients with CSAP underwent 17 ablation procedures and 10 patients with RPS pathways underwent 10 ablations. There was no difference in demographic data or energy source for ablation $(\mathrm{p}=0.2)$. Comparison of pre-ablation 12-lead ECGs demonstrated pre-excitation in $8 / 10$ $(80 \%)$ patients with RPS pathways versus 9/13 $(69 \%)$ patients with CSAP $(p=0.46)$; however, pre-excitation with a deep Q wave in lead II was seen in 5/9 (56\%) patients CSAP versus 0/8 in RPS $(\mathrm{p}=0.07)$. Accessory pathway (AP) potentials, defined as either a sharp potential or continuous low amplitude, high frequency signal between the 
$\mathrm{A}$ and $\mathrm{V}$ electrograms, were seen on the CS catheter in 6/17 (35\%) of CSAP ablations and in 0 RPS ablations $(\mathrm{p}=0.041)$. AP potentials were seen at the site of successful ablation in 11/17 (65\%) CSAP ablations but only in 2/10 (20\%) RPS pathways $(\mathrm{p}=0.027)$. CS diverticulum was identified in $2 / 13$ (15\%) patients with CSAP; CS angiography was not routinely performed for RPS ablation. Acutely successful sites for ablation of CSAP included: 9 in neck of middle cardiac vein, 3 in middle cardiac vein, 1 in neck of lateral cardiac vein, 1 in neck of posterior cardiac vein, and 3 in neck of CS diverticulum. There was no difference in timings on the His, CS or ablation catheters in NSR, V-pacing or free-running SVT. Recurrence of tachycardia occurred in $26 \%$ of patients with CSAP and $10 \%$ of patients with RPS pathways $(\mathrm{p}=0.18)$. Conclusions: Epicardial pathways, while an uncommon mechanism of pediatric SVT, should be considered when pre-ablation ECG demonstrates pre-excitation with a Q wave in lead II and if an AP potential is seen on the CS catheter. CSAP can exist despite the absence of a macroscopic CS diverticulum. Failure to eliminate tachycardia after RPS ablation or the need for multiple ablations should raise suspicion for an epicardial pathway.

Correspondence to Edward Rhee: edward.rhee@chw.edu

\section{A prospective cohort study of arrhythmias in the neonatal intensive care unit}

Edisa Tokovic, Nadia Badrawi, Ranya Hegazi, Wael Lotfy, Fadya Mahmoud, Hany Aly

The Children's National Medical Center, Washington, DC, USA and Cairo Children's Hospital, Cairo, Egypt

Background: Arrhythmias among newborns are not uncommon, but the exact incidence and types of arrhythmia in the neonatal intensive care unit (NICU) is not known. It is also not fully clear whether different medical conditions, interventions and medications administered to these ill infants could predispose to arrhythmias. In this study we aimed to identify the incidence, common types, associated risk factors and presentations for arrhythmia in the NICU. Methods: We prospectively conducted 12-lead EKG studies on a random sample of 457 neonates who were $>3$ days old and $>28$ weeks gestation. All Infants were evaluated for the presence of arrhythmias. A 24-hour Holter monitoring was offered randomly to every 4th baby with normal EKG and to all of those who demonstrated arrhythmias on their EKG creating 3 groups: normal, benign and non-benign arrhythmia. Two-dimensional echocardiography (ECHO) was performed in all neonates clinically assessed with murmurs or electrophysiologically diagnosed with arrhythmia. Screening results were correlated with maternal, obstetrical and neonatal data. Unpaired student t-test, Mann-Whitney test, Chi square test, Fisher's exact and analysis of variance testing were used for analysis. Correlation and regression analyses were also performed whenever appropriate. Results: Of the 457 patient EKGs studied, 39 (8.5\%) had benign arrhythmia, and $7(1.5 \%)$ had non-benign arrhythmia. Male gender, maternal age and maternal smoking were identified as risk factors for arrhythmia. Infants with benign arrhythmia had lower glucose levels (89 $38 \mathrm{mg} / \mathrm{dl}$ vs. $10329.2 \mathrm{mg} /$ $\mathrm{dl}$ in the normal group, $\mathrm{p}=0.03$ ). A total of 139 infants had Holter monitoring, with 48 infants showing arrhythmia. None of the infants with arrhythmias were $<32$ wks, $12(25 \%)$ were $32-37 \mathrm{wks}$, and 36 (75\%) were $>37 \mathrm{wks}$. Arrhythmia significantly correlated with severe hypoxic ischemic encephalopathy $(p=0.03)$, high umbilical artery lines $(p<0.01)$, and beta- 2 receptors agonist drugs used for nebulization $(\mathrm{p}=.023)$. Umbilical venous lines and dopamine infusion were not associated with arrhythmia. Conclusions: Arrhythmias have higher prevalence among NICU population compared to previously reported figures in neonates. They are more common in male infants with older gestational age. Maternal smoking, high umbilical artery lines, lower glucose levels, and the use of the nebulized beta- 2 adrenergic treatment were associated with benign arrhythmia.

Correspondence to Edisa Tokovic: etokovic@cnmc.org

\section{Caregiver anxiety upon discharge for neonates with congenital heart disease}

Abigail Fischer, Catherine Butz, Angela Blankenship, Lisa Nicholson, Peter Dyke, Clifford Cua

\section{Nationwide Children's Hospital, Columbus, OH, USA}

Background: Parents of children with congenital heart disease (CHD) have been shown to be at an increased risk of having psychosocial morbidities including anxiety, depression, and somatization. However, little is known about the anxiety level of caretakers of neonates with CHD during the initial hospital course. The goal of this study was to evaluate the anxiety level of the guardians of neonates with $\mathrm{CHD}$ at time of hospital discharge 
and to determine if certain characteristics predict higher anxiety levels. Methods: Caregivers of neonates admitted with CHD within the first 30 days of life were recruited. Anxiety levels were measured by using the Spielberger State-Trait Anxiety Scale. This scale, in addition to a demographic questionnaire were administered 24 to 48 hours prior to hospital discharge. T-tests were used to determine significant differences among means between parents who were clinically anxious and those who were not. Bivariate and multivariate linear regression was used to evaluate statistical correlations among variables. Results: Fiftynine questionnaires were completed for 37 neonates. Thirty-five questionnaires were completed by females. Average age of the caregivers was $28.3+5.8$ years and age of the infant at time of hospital discharge was $24.5+22.1$ days. Eight neonates had single ventricle physiology. Twenty-four neonates had an intervention performed before discharge. Based on the state score measure of anxiety, $81 \%$ of caregivers denied anxiety, $13.6 \%$ were borderline anxious, and 5\% reported clinically significant levels of anxiety. Similarly, trait scores of anxiety reflected $93 \%$ of caregivers who denied anxious symptoms, $1.7 \%$ reported borderline anxiety, and 5\% reported a clinically significant level of anxiety. There was a significant correlation between state score and level of education $(r=0.33, p<0.05)$ and the trait score and level of education $(r=0.29$, $p<0.05)$. There was an inverse relationship between number of medications and trait score $(\mathrm{r}=-0.27$, $\mathrm{p}<0.05)$. The level of education explains $9 \%$ of the variance in state score and level of education and number of medications explain $13 \%$ of variance in trait score. There were no other significant correlations between state or trait anxiety and baseline demographics/characteristics. There were no significant differences in anxiety scores between mothers and fathers. Conclusions: Surprisingly, caregiver anxiety was overall low for neonates with CHD, although this may have been due to timing of assessment just prior to hospital discharge. Increased level of education was associated with higher anxiety levels. Future studies are needed to better evaluate this population with the goal of identifying possible factors of family dynamics and hospital course that may keep anxiety to a minimum for caregivers of these complex neonates.

Correspondence to Clifford Cua: clcua@hotmail.com

\section{Quality of life and school re-entry problems in pediatric heart transplant (HT) patients}

Marsha Ayzen, Juan Alejos, Donald Barr, David Teitel, Paul Pitlick
UCLA Mattel Children's Hospital, Stanford

University, University of California, San Francisco, CA, USA

Background: Well-established support programs are currently in place to help pediatric oncology patients in their transition back to school. However, few of these programs extend their services to children who have undergone other major medical interventions such as heart transplantation. We hypothesized that patients undergoing heart transplantation have less problems related to their school re-entry then do oncology patients secondary to less chronicity of their illness. The purpose of this study is to define the quality of life and impact on schooling of HT patients in comparison to that of the pediatric oncology patients. Methods: To date, twenty-one pediatric oncology patients and ten pediatric HT patients have been enrolled in the study. Surveys administered each patient and their parents were used to evaluate demographics, quality of life, and perceptions of school before and after treatment. Quality of life and perceptions of school were graded by the five point Likert scale and qualitative responses. Descriptive statistics, independent sample t-tests, and correlations were used to analyze data. Results: Reduction in quality of life and school re-entry difficulties were identified as significant problems in both HT and oncology patients. No differences were found in the quality of life or school related measures between $\mathrm{HT}$ and oncology patients $(\mathrm{p}>0.1)$. Significant improvements were found in quality of life after treatment $(\mathrm{p}<0.05)$ and school re-entry measures after reentry $(p<0.1)$ in all study patients who attended private schools versus those who attended public schools. Qualitative responses showed that both children and parents considered school re-entry an important but difficult transition and valued the help they received from the schools and/or the hospital. Conclusions: Survey-based data indicates that both pediatric oncology and HT patients struggle with post-treatment quality of life and school re-entry. Our hypothesis that HT patients would fare better than oncology patients because of less chronicity of illness was not supported. However, school re-entry was affected by school type, public vs. private, suggesting that other factors can ameliorate this stress and anxiety. This becomes crucial when it is seen that HT patients do not have formalized support systems similar to those now in place for the oncology population.

Correspondence to Marsha Ayzen: ayzen@email.chop.edu 


\section{Ventricular stroke work by three- \\ dimensional echocardiographic pressure : volume loop analysis}

\author{
G. Hamilton Baker, Girish Shirali, Jeremy \\ Ringewald, Tim McQuinn
}

\section{Medical University of South Carolina, Charleston,} SC, USA

Background: Ventricular pressure:volume loop (PVL) analysis provides valuable indices of myocardial pump function. We used a novel method of PVL analysis combining simultaneous 3-dimensional echocardiography (3DE) and catheterization data to measure ventricular stroke work. We hypothesized that stroke work calculated by this method would correlate highly with non-invasive estimates of stroke work. Methods: PVLs were constructed in 8 patients, 5 in whom PVLs were acquired before and after catheter intervention. 3DE left ventricular (LV) full volumes were acquired with simultaneous LV pressure recording by fluid-filled catheters (Series IV, WITT Biomedical). LV volume throughout the cardiac cycle was calculated with volumetric software (3DQ Adv, Philips Medical). PVLs were constructed by synchronizing volume and pressure data using the electrocardiogram. Stroke work (SW) was calculated as the area within the PVL. Non-invasive SW was calculated as the product of mean arterial pressure by cuff and 3D stroke volume. Results: The patients' diagnoses included: PDA $(n=4)$; atrial septal defect $(n=2)$; aortic stenosis $(n=1)$; coarctation of the aorta $(\mathrm{n}=1)$. The median patient age was $9.6 \mathrm{yr}$ (0.25 $\square$ 14). Median 3D - LV end diastolic volume $=25 \mathrm{ml}(5.3 \square$ 133.8), median $3 \mathrm{D}-\mathrm{LV}$ end systolic volume $=12.9 \mathrm{ml}(1.3 \square 71.5)$, and median 3D - LV ejection fraction $=54 \%(46.6 \square$ 76.4). By PVL, the median LV SW $=1243 \mathrm{~g} * \mathrm{~cm}$ $(523-3719 \mathrm{~g} * \mathrm{~cm}) ;$ mean non-invasive $\mathrm{SW}=$ $1192 \mathrm{~g} * \mathrm{~cm}(249-3587 \mathrm{~g} * \mathrm{~cm})$. Spearman correlation coefficients calculate for SW and non-invasive stroke work was $0.98(\mathrm{p}<0.001)$. The mean difference by Bland-Altman analysis (+2SD) between SW and non-invasive $\mathrm{SW}=210+490 \mathrm{~g} * \mathrm{~cm}$. Conclusions: These preliminary data demonstrate that PVL analysis by $3 \mathrm{DE}$ and catheterization is feasible and provides the potential to calculate useful indices such as SW. SW measured by PVL demonstrated excellent correlation and good agreement with non-invasive estimates. Combination of this novel method of PVL analysis with controlled load alteration may provide a clinically practical method for analysis of contractility.

Correspondence to G. Hamilton Baker: baker@musc.edu

\section{Unsuspected coronary arterial anomalies found during 64-detector multi-slice CT evaluations of congenital heart disease}

James Mathewson, Stephen Pophal, John Nigro, J. Hauschildt, F. Ing, J. Lamberti

Eller Congenital Heart Center, St. Joseph's Hospital and Medical Center, Phoenix, AZ, USA and Rady Children's Heart Institute, Rady Children's Hospital, San Diego, CA, USA

Background: The purpose of this study was to describe serendipitously discovered coronary artery anomalies and their effect on patients management using 64-detector multi-slice CT (64MSCT) performed in patients with known or suspected congenital heart disease (CHD). Methods: We reviewed the findings of 107 64MSCT studies performed in 105 patients with $\mathrm{CD}$ at a single tertiary pediatric center from August 205 through April 2006. In every patient, the indications for study related to known or suspected CHD. Results: Of 20 patients who had coronary anomalies (19\%), 14 were unsuspected. Patient age ranged from 4 days to 25 years (median 6.3 years). Heart rate ranged from 60 to $163 \mathrm{bpm}$ (median $100 \mathrm{bpm}$ ). All were ECG-gated. Anomalies included aneurysms (2) anomalous origin of the left coronary artery from the right pulmonary artery (1), high take-off of the right coronary artery from the ascending aorta between the main pulmonary artery and the aorta (3), anomalous origin of the circumflex coronary artery from the right coronary artery (2) and from a separate orifice from the right sinus (1), single coronary artery ostium from the posterior sinus (2), 3 separate coronary ostia (2) origin of the conus branch from the left coronary artery in 1 patient with bilateral right ventricles, and absence of the left main with supply of the left anterior descending and circumflex coronary arteries from a right conus branch (12). Nine (64\%) had had prior cardiac catheterization, and all had had prior twodimensional echocardiography. As a direct result of 64MSCT, management plans were altered in 4 of these 14 patients (29\%). Conclusions: 64MSCT may serendipitously discover previously unknown coronary artery pathology. Due to the high incidence of coronary anomalies in patients with CHD, 64MSCT may prove to be an effective adjunct to traditional imaging modalities for defining coronary anatomy in these patients.

Correspondence to James Mathewson: jwmathewson@yahoo.com 


\section{Prevalence of undiagnosed structural cardiac anomalies in children undergoing echocardiography for Kawasaki disease}

Annette Baker, David Fulton, Jami Levine, Jane Newburger

\section{Children's Hospital, Boston, MA, USA}

Background: The prevalence of congenital heart disease is cited as 8 per 1000 live births, but few studies have examined the prevalence of structural anomalies in the general population of infants and children without previously identified heart disease. Methods: We performed a retrospective review in children without known heart disease who underwent 2-D echocardiography (echo) during evaluation for possible Kawasaki disease (KD) at a single institution from 1981-2007. Results: Of 2069 pts undergoing 2-D echo for the indication of KD, 21 had known pre-existing congenital heart disease (1\%), and 2 were referred from other centers for transcatheter closure of patent ductus arteriosus (PDA) discovered during echo for KD. The remaining 2046 patients were not known to have structural cardiac anomalies prior to echo at our center and constitute our study cohort. Of these, $61 \%$ were male. Overall, 52 pts $(2.54 \%)$ were found to have structural cardiac anomalies of the following types: bicuspid aortic valve in 8 pts $(0.39 \%)$; ventricular septal defects, all small muscular, in 3 pts $(0.15 \%)$; PDA in 14 (0.68\%); atrial septal defects (ASD) of at least moderate sized with right ventricular volume overload in $5(0.24 \%)$; a congenital anomaly of the mitral valve in $1(0.05 \%)$, and mitral valve prolapse (more than trivial) in $5(0.24 \%)$. Coronary artery (CA) anomalies were seen in 16 pts $(0.78 \%)$ including 5 with anomalous origin of the RCA from the L sinus of Valsalva with intramural course between the great arteries and 11 benign coronary artery variants, 2 with single right coronary. To date, 5 pts $(0.24 \%)$ have had interventions: 2 pts $>$ age $5 \mathrm{yrs}$ had closure of moderate to large ASDs (1 device, 1 surgical); 2 pts had PDA closure (1 coil, 1 VATS ligation); and 1 pt underwent unroofing of anomalous intramural RCA. The remaining 4 pts with anomalous RCA from L sinus of Valsalva with intramural course are being followed and may require surgery in the future. Conclusions: Important structural cardiac defects are found in infants and children without previously known heart disease during echo evaluation for KD. Further studies should explore the feasibility of cost-effective echo screening in children, particularly as they enter competitive sports.

Correspondence to Annette Baker: annette.baker@cardio.chboston.org
Associated symptoms at presentation in Kawasaki disease

Annette Baker, M. Lu, L. L. Minich, A. M. Atz, G. Klein, R. Korsin, L. Lambert, J. S. Li, W. Mason, E. Radojewski, V. L. Vetter, J. W. Newburger

\section{For the Pediatric Heart Network, USA}

Background: In addition to the diagnostic criteria for Kawasaki Disease, affected children may have nonspecific associated symptoms. Few prospective data have been collected on their prevalence. The purpose of this report is to describe common associated symptoms among subjects enrolled in the Pediatric Heart Network's trial of steroid therapy in KD. Methods: Patients (pts) who met study criteria for acute Kawasaki disease were enrolled between days 4 and 10 of illness at 8 centers in North America between 2002-2004. A medical history was obtained at the time of presentation, and all symptoms were coded. We defined common associated symptoms as those occurring in $>10 \%$ of patients within the 10 days prior to presentation. Principal clinical criteria for diagnosis of Kawasaki Disease were not included in this analysis. Results: Of 199 pts enrolled, 1 withdrew prior to treatment, and data were analyzed for 198 pts. Within 10 days of presentation, a history of irritability was reported in 98 pts $(50 \%)$, vomiting in 88 (44\%), decreased food/fluid intake in $73(37 \%)$, cough in $55(28 \%)$, diarrhea in $52(26 \%)$, rhinorrhea in $37(19 \%)$, weakness in 37 $(19 \%)$, abdominal pain in $35(18 \%)$, and joint pain (arthralgia or arthritis) in $29(15 \%)$. One or more gastrointestinal symptom (vomiting, diarrhea, or abdominal pain) was present in 120 pts $(61 \%)$ and 69 pts $(35 \%)$ had $\geqslant 1$ respiratory symptom (rhinorrhea or cough). Higher ALT at admission was associated with vomiting $(p=0.03)$ and abdominal pain $(\mathrm{p}=0.01)$. Lower $\operatorname{Ig} \mathrm{A}$, adjusted for age, was associated with diarrhea $(\mathrm{p}=0.03)$. Younger pts were more likely to have irritability and rhinorrhea $(\mathrm{p}<0.001$ and $\mathrm{p}=0.03$, respectively), whereas older pts were more likely to have vomiting $(\mathrm{p}=0.01)$, abdominal pain $(\mathrm{p}<0.001)$, headache $(\mathrm{p}=<0.001)$, stiff neck $(\mathrm{p}=0.01)$, and joint pain $(\mathrm{p}=0.02)$. Conclusions: Nonspecific symptoms occur commonly in children with Kawasaki Disease. To reduce delays in the diagnosis, clinicians should be educated that such symptoms may comprise a significant component in the chief complaint.

Correspondence to Annette Baker: annette.baker@cardio.chboston.org 
Sixty-four detector row multi-slice CT: initial evaluation of its role in diagnosis and management of congenital heart disease

James Mathewson, Stephen Pophal, John Nigro, J. Hauschildt, F. Ing, J. Lamberti

Eller Congenital Heart Center, St. Joseph's Hospital and Medical Center, Phoenix, AZ, USA and Rady Children's Heart Institute, Rady Children's Hospital, San Diego, CA, USA

Background: The role of 64-detector multi-slice CT (64MDCT) to assess congenital heart disease (CHD) has not been fully defined. The purpose of this study was to describe our initial experience using 64MDCT to diagnose and manage patients with CHD. Methods: We reviewed the findings from 107 64MDCT studies performed on 104 patients with CHD from August 2005 through April 2006. Results: Injection rates ranged from 1-5 cc/sec. 96 were ECG-gated. 47 had prior cardiac catheterization (CATH). All had prior 2D echocardiography (2DECHO). Patient age ranged from 1 day to 61 years (median 8.7 years). Heart rate ranged from 43 to $171 \mathrm{bpm}$ (median 96). We evaluated: atrial and ventricular septal defects (3), tetralogy of Fallot (6), double outlet right ventricle (5), transposition (7), aortic coarctation (11), truncus arteriosus (1), interrupted aortic arch (5), single ventricle (17), pulmonary venous obstruction (3), vascular ring/ sling (9), origin/size of branch pulmonary arteries (30), pulmonary artery bland position and tightness (4), stent/shunt patency and adjacent aneurysm (6), coronary artery origin/size, aneurysms (20), complete AV Canal (2), Williams's Syndrome (4), and the position of the cardiac structures underlying the sternum in all. In addition, RV and LV ejection fraction were measured (57), and LV wall thickness and shape assessed (2). The atrial and ventricular septa, trachea, lungs, and air-filled esophagus were examined in all. The coronary arteries were visible in every patient. We found 53 instance of unknown and unsuspected pathology in 32 patients. In a few cases image quality was adversely affected by inappropriate bolus timing, beam hardening artifacts, chest wall movement, and irregularities of rate and rhythm. As a result of 64MDCT, patient management was modified in $45(43 \%$. There were no complications or errors in diagnosis. Conclusions: $64 \mathrm{MDCT}$ is a viable imaging modality for examining $\mathrm{CHD}$, determining ventricular systolic function, defining coronary artery anatomy, and visualizing the airway and lungs. 64MDCT depicts all pathology within a region of interest, is a rapid and safe imaging modality for evaluating CHD, and may serve as an alternative to $\mathrm{CATH}$ and an adjunct to $2 \mathrm{DECHO}$ and MRI.

Correspondence to James Mathewson: jmathewson@yahoo.com

\section{Research in the cardiac operating room: nursing knowledge, communication and sample collection}

Michele Barrila, Diane Hartman

\section{Children's Hospital of Philadelphia, Philadelphia, PA, USA}

Background: The number of pediatric research studies which require sampling of blood or tissue in the cardiac operating room (OR) continues to increase. Frequently, cardiac research studies include acquisition of blood or tissue samples perioperatively for assessment of protein levels, genetic polymorphisms, drug levels, or other factors. In addition, randomization for some treatment trials must be performed in the OR. Thus communication must be flawless to ensure the appropriate procedure is completed and samples obtained. Poorly designed processes will create poor communication between the OR and research staff which will impede accurate specimen collection or appropriate randomization. This type of error could compromise study completion or possibly increase the patient risk of an adverse outcome. The goal of the study is to design a system that ensures that the right research samples are collected on the correct population, using the appropriate methods, to minimize blood loss, and preserve sample integrity for optimal research data collection. Methods: Steps of the current communication process for studies which require sampling or randomization were evaluated. Areas where errors might occur were identified and assessed for severity and frequency of occurrence. The OR nursing staff's knowledge of current ongoing research and motivation for research facilitation was also evaluated. Results: Currently six active research studies require sampling or randomization in the cardiac operating room (blood sample only $(n=4)$, blood \& tissue sample $(n=1)$ randomization $(n=1))$. A six month period, between October 1, 2006 \& March, 31 2007, was evaluated for success rate of blood and tissue sample collection. Successful sample collection varied among the different studies, and ranged from $88.7 \%-100 \%$ (median 96.4\%). Studies with research personnel present in the operating 
room had a $100 \%$ success collection rate, but where research personnel were absent, $4 \%$ to $12 \%$ (median $6 \%$ ) percent of the time samples were missed or collected inaccurately. A survey of the OR nursing staff revealed that only $38.5 \%$ of the time the staff could correctly identify the principle investigator, research coordinator and specific preparation of research sample for the appropriate research study. Conclusions: Presence of research personnel in the OR was associated with improved success in study completion. An action plan was implemented in August 2007 to improve staff education, motivation and communication in order to improve the sample collection rate, even when dedicated research personnel can not be present. New processes are being implemented to improve facilitation and completion of all research interventions. Evaluation of these new processes is ongoing.

Correspondence to Michele Barrila: barrila@email.chop.edu

Transitory $T$ wave inversion in left chest leads in healthy children and adolescents

Lidija Vucajnk, Igor Vucajnk

Pediatric Department, General Hospital Celje, Slovenia

Background: T wave represents part of ventricular repolarisation and should be positive in left chest leads in children and adolescents. Negative $T$ wave in left chest leads could be a sign of different pathological states, such as: left ventricular hypertrophy, myocardial ischemia or infarction, myocarditis, pericarditis, cardiomyopathy, long QT syndrome, WPW syndrome, heart tumor, LBBB, mitral valve prolapse, posttachycardia syndrome, Wellens' syndrome, acute pulmonary embolism, cerebrovascular accidents (especially intracranial bleeds), tuberous sclerosis, anorexia, poisoning with $\mathrm{CO}$ or arsenic, Friedreich's ataxy or myopathies, but could be also idiopathic, probably as a result of increased sympathic tone. The aim of this study is to find out the incidence of inverted $\mathrm{T}$ waves in left chest leads in healthy children and adolescents. Methods: In 269 children aged from 5 to 18 years 24-hours ECG Holter monitoring was performed. There were 179 girls and 90 boys with no history of taking any medication, with normal somatic and neurological examination, normal 12-leads surface ECG and echocardiography and normal results of electrolite, glucose, muscle enzyme, troponin and thyroid hormones, with no arterial hypertension, no eating disorders and no history of recent infection.
Results: Among 269 healthy children in 13 (4.8\%) were found to have transitory inverted $\mathrm{T}$ waves on 24-hours ECG Holter monitoring. Transitory inverted $\mathrm{T}$ waves were seen during the normal daily activity, overnight sleep and/or during the exercise. They were found in children aged from 11 to 18 years (mean 15.2 years) and were more often in boys (7.7\% in boys, $3.3 \%$ in girls). $\mathrm{T}$ waves were negative in I,II,III, aVL, aVF and V3-6 and positive in aVR and V1, varied in V2-3. There were no ST segment changes. During the inversion of $\mathrm{T}$ waves children were asymptomatic. Conclusions: Transitory $T$ wave inversion in left chest leads are occasionally seen in healthy children and adolescents on 24-hour ECG Holter monitoring. The incidence is low. Before notifying this condition as idiopathic it is advisable to exclude pathologic causes of the inversion.

Correspondence to Lidija Vucajnk: lidija.vucajnk1@guest.arnes.si

\section{Aortic root measurements in pediatric patients: magnetic resonance imaging versus echocardiography}

Wasim Khan, Frederick K. Emge, Fareed Ahmad

\section{Geisinger Medical Center}

Background: Aortic root dimensions can be readily measured using 2-dimensional echocardiography. This has been correlated with intraoperative direct measurements. This modality has become the most common method for monitoring aortic root dimensions during growth. Evaluation of the aortic root has also been standardized to body surface area in children and adults. MRI is a non-invasive modality that is fast gaining clinical acceptance for cardiovascular evaluation, especially in adults. However, no comparisons of magnetic resonance imaging have been made with the current "gold standard" of echocardiography, particularly in children. Methods: A retrospective review was performed on the images of the 65 children, less than 18 years of age, who underwent cardiac MRI and ECHO at our institution. Patients were excluded if their MRIs did not include imaging of the left ventricular outflow tract and ascending aorta. Ages ranged from 6 months to 17 years. MR imaging was performed using a 1.5-T GE CVi clinical MR scanner. For assessment of aortic root measurements, FIESTA (Fast image employing steady state acquisition) short axis, radial, and "candy cane" images were obtained through the aortic root. Standard parasternal long axis echocardiography imaging was 
performed to obtain these measurements in the identical places. Measurements were performed at the annulus, sinuses of Valsalva, sino-tubular junction, and proximal ascending aorta. Clinicians interpreting the echocardiograms and MRI images were blinded as to these results. Results: The results were analyzed using a paired t-test. The aortic annulus measurement from MR was $0.19 \mathrm{~mm}$ smaller than ECHO. This was not significant with a $\mathrm{p}$ value of 0.45 . The measurements at the sinus of Valsalva for MRI were $0.36 \mathrm{~mm}$ larger than echo and also not significant, with a p-value of 0.057 . Measurements at the Sino-tubular junction were $0.31 \mathrm{~mm}$ larger than echo and not significant with a $\mathrm{p}$ value of 0.06 . And the measurements at the proximal ascending aorta for MRI was $0.34 \mathrm{~mm}$ smaller than ECHO which was statistically significant with p-value of 0.047. Conclusions: Limitations of echocardiography in acquiring the ascending aorta measurements is a well understood issue. This is especially true in the older patient or the child with chronic lung or cardiac disease, where limited acoustic windows hamper discrete aortic imaging. However, MRI may pose different limitations, which relate to obtaining the proper oblique angle to best assess the diameter of the proximal ascending aorta. The lack of correlation based on this comparative study at this measurement site may reflect either or both of these modality related issues. However, there is good correlation with the other measurement sites. MRI is a powerful tool in pediatric cardiovascular imaging, giving both anatomic and physiologic information. Though this study is a small sample size, it does support close correlation between cardiac MR and echocardiographic measurements in the same individual. Cardiac MR is a safe and accurate method for aortic root measurements, specially in patients with limited acoustic windows for echocardiography.

Correspondence to Wasim Khan: wkhan1@geisinger.edu

\section{Recruitment challenges in studies of long-term outcome}

Nancy Burnham, Diane Hartman, Courtney Schwalbe, Jennifer Raue, Jennifer Hufford, Gil Wernovsky, J. William Gaynor

The Children's Hospital of Philadelphia, Philadelphia, PA, USA

Background: With improved early survival, longterm outcome of children with congenital heart disease (CHD) requiring surgical interventions are of increasing importance to both families and professionals involved in their care. Long-term outcome studies are both time consuming, costly, but necessary. Between 1998-2003, 550 infants with CHD were prospectively enrolled in a clinical research study to evaluate the association of APOE genotype with neurodevelopmental dysfunction. Between 1999-2003, 360 (72\%) returned for half a day developmental testing at one-year of age. Beginning in 2003, this same cohort aged 4 years were eligible to for a full day of developmental testing including an assessment of cognition, behavior, speech and language, hearing, and motor abilities. Initial recruitment efforts demonstrated a $48 \%$ return rate, not as robust as anticipated. A majority of families had not been contacted since the one year follow-up. Military families posed a particular challenge. There were predictable family needs which we continued to see throughout the entire time period of the study including sibling childcare, workplace issues, and transportation. The purpose of this review is to describe our experiences specifically in regards to recruitment challenges and our responses to those challenges as clinical research nurse coordinators resulting in successful enrollment in large outcome study. Methods: A retrospective review of the enrollment data and recruitment challenges between 2003-2007 was performed. Results: Initially 2 RNs with a combined 20 years of pediatric cardiac nursing experience and 2 years of research experience initiated recruitment. Our cardiac research nurse team doubled in size by the third year allowing us to provided consistent attention to recruitment strategies. Interestingly, the best return rates occurred during the third and fourth grant years. Family contact materials consisted of a variety of documents sent at several points in time. Several webbased people locaters such as Lexis-Nexis were used to verify demographic information. The initial mailing sent the month of their child's 4th birthday included a letter, brochure, consent form, and a bookmarker with their child's name and a birthday greeting. After re-establishing contact, we made efforts to maintain updated information by collecting alternative contact information at the time of their study visit. As of 10/31/07, 94\% of the eligible cohort $(n=486)$ has been contacted by the research coordinator group and $370(76 \%) 4 \mathrm{yr}$ olds and their families have returned for a day of developmental testing. The enrollment rates for the 5 grant years have been $58 \%, 81 \%, 87 \%, 81 \%$, $77 \%$ (to date) respectively. Families have returned from a variety of geographic regions: $67 \%$ located in our immediate region (PA,NJ,DE); $22 \%$ from our 
next largest referral area (VA,MD,NC,WV), $11 \%$ from the Midwest, FL, MS, West Coast and overseas. We also tested 17 sets of twins and triplet pairs. Conclusions: Re-contacting and enrolling patients and their families for long-term follow-up studies pose many challenges. A variety of strategies are useful to locate families and maintain interest in the study. Ideally patients enrolled prospectively in studies will be contacted at regular intervals to maintain contact, update information, and provide new information to families.

Correspondence to Nancy Burnham: burnhamn@email.chop.edu

\section{Long-term outcome after repair of tetralogy of Fallot with absent pulmonary valve}

Kouta Agematsu, Hiromi Kurosawa, Kazuaki Ishihara, Takahiko Sakamoto, Yusuke Iwata, Noboru Yamamoto, Goki Matsumura, Minori Tateishi, Hironobu Morimoto, Masahiro Ohkura, Tai Fuchigami

\section{Tokyo Women's Medical University, Tokyo, Japan}

Background: Tetralogy of Fallot (TOF) with absent pulmonary valve (APV) syndrome is a rare cardiac anomaly and often reveals severe respiratory distress due to compression of dilated pulmonary artery to the trachea and bronchus. Surgical mortality is still high, particularly in infant patients presenting with severe respiratory compromise. Methods: We reviewed 24 consecutive patients who underwent definitive repair of TOF/APV between 1983 and 2003 (19 patients in Tokyo Women's Medical University, 5 patients in Chiba Children's Hospital). Fifteen patients $(63 \%)$ showed repeated respiratory distress symptom and 7 patients (29\%) were dependent on ventilator before operation. Mean age and body weight at operation were $3.00 \pm 3.95$ years (0.08-12.46) and $11.4 \pm 10.4 \mathrm{~kg}(3.6-42.0)$, respectively. Results: All patients underwent ventricular septal defect patching. Seventeen patients $(71 \%)$ underwent pulmonary artery plication at the time of definitive repair. Right ventricular outflow tract (RVOT) was reconstructed with hand-made valved conduit in 3 patients (13\%), monocuspid or tricuspid transannular patch in 21 patients $(87 \%)$. There were two hospital deaths due to respiratory failure and heart failure, there were three late deaths; gastrointestinal bleeding after repeated respiratory distress (1) and unknown reasons (2). Actuarial survival by Kaplan-Maier curve was $83 \%$,
$79 \%, 79 \%$ at 1,5 and 10 year respectively. In nonsurvivors, three patients had prolonged respiratory distress after the operation and autopsy or tracheal fiber scope findings showed tracheal malacia, hypoplastic trachea and obstructive lung disease. In survivors the postoperative echocardiogram and cardiac catheterization showed good right ventricle function: mean RVEF of $5318 \%$ but moderate pulmonary valve regurgitation was recognized in 12 patients $(80 \%)$. Freedom from re-operation 1,5 and 10 years after definitive repair was 100,94 and $78 \%$, respectively. Re-operation for RVOT was necessary in three patients, in whom initial RVOTR was performed with hand-made conduit (2) and with transannular patch (1). RVOTR with extracardiac hand-made conduit increased rate of re-operation $(\mathrm{p}<0.01)$. In long-term period, Chest X-ray revealed cardio-thoracic ratio of $57.916 .9 \%$ and 16 patients (89\%) showed NYHA class I, II and 1 patient (6\%) showed NYHA class III. Conclusions: The surgical outcome for infant TOF/APV patients who showed severe respiratory symptom was unsatisfactory. As the next strategy, identifying airway pathology and the treatment for respiratory tract might be necessary before and during the definitive repair in the high risk $\mathrm{TOF} / \mathrm{APV}$ patients. Re-operation rate is relatively low in our institute but Re-RVOTR might be necessary due to pulmonary valve insufficiency in the future.

Correspondence to Kouta Agematsu: agematsuagain@yahoo.co.jp

The effects of surgically induced right bundle branch block on left ventricular function after ventricular septal defect closure

Thais A. Pedersen, Niels H. Andersen, Mette R. Knudsen, Thomas D. Christensen, Keld E. Sorensen, Vibeke E. Hjortdal

Aarbus University Hospital, Skejby, Aarbus, Denmark

Background: Closure of VSD is the most frequent open-heart procedure in children. Surgically induced RBBB is seen in up to $60 \%$ and chronic depolarization abnormalities may over time theoretically impair left ventricular function. The aim of this study was to determine the long-term significance of right bundle branch block (RBBB) on left ventricular systolic and diastolic function in children operated for ventricular septal defect (VSD). Methods: Twenty-six children with normal preoperative ECG who underwent VSD closure through an atriotomy were studied $11 \pm 2$ years postoperatively. Fourteen $(54 \%)$ developed RBBB postoperatively. To determine systolic and diastolic properties of the left ventricle, conventional 
and tissue Doppler echocardiography were performed. Twenty-six gender and age matched healthy children served as controls. Results: Irrespective of the presence of $\mathrm{RBBB}$, the peak systolic velocity of the septal mitral ring was lower in the surgical subgroups (RBBB $5.2 \pm 1.4 \mathrm{~cm} / \mathrm{s}$, non-RBBB $5.4 \pm 1.2 \mathrm{~cm} / \mathrm{s}$, vs. control group $6.6 \pm 1.0 \mathrm{~cm} / \mathrm{s}, \mathrm{p}<0.01)$. Regarding the diastolic function, the septal $\mathrm{E} / \mathrm{E}^{\prime}$ ratio was significantly higher in children with RBBB $(12 \pm 3.0 \mathrm{~cm} / \mathrm{s}$ vs. control group $8.4 \pm 1.5 \mathrm{~cm} / \mathrm{s}$, $\mathrm{p}<0.01$ ), but not significantly higher in children without RBBB compared to the control group $(10.6 \pm 4.0 \mathrm{~cm} / \mathrm{s}$ vs. $8.4 \pm 1.5 \mathrm{~cm} / \mathrm{s}, \mathrm{p}=0.13)$. The fractional shortening percentage was similar in all three subgroups. The changes in left ventricular function were not significantly related to age at surgery, follow-up period or surgical method (patch/ suture). Conclusions: Systolic long axis function is significantly reduced in VSD operated children irrespective of the presence of RBBB. However, diastolic dysfunction seemed primarily related to children with post-operative RBBB.

Correspondence to Thais A. Pedersen: thais.a.pedersen@ki.au.dk

\section{Development of an interprofessional pediatric ventricular assist device support team}

Sarah Furness, Cecelia St. George-Hyslop, Barbara Pound, Misty Earle, Andrea Maurich, Danika Rice, Tilman Humpl

\section{Sick Kids Hospital, Toronto, ON, Canada}

Background: The Berlin Heart EXCOR. Pediatric Ventricular Assist Device (VAD) was introduced to SickKids Hospital, Toronto in 2004, to support critically ill children in heart failure, as a bridge to cardiac transplantation. Caring for paediatric patients with a VAD, requires a collaborative approach from an interprofessional team to ensure maximum patient safety and optimal outcomes. Initiating a VAD program is challenging, due to the complex medical and technical nature of this device, and associated learning needs. Consideration of varied roles and responsibilities of each interprofessional team member involved in the care is critical. Methods: In 2004 seven team members of the SickKids cardiac program travelled to Berlin to learn about the Berlin Heart from the German Heart Centre, a well established centre for VAD care. The core VAD team was made up of a cardiac intensivist, cardiac critical care nursing staff and a nurse educator. Their responsibilities included educating involved health care professionals, patients and families about the VAD. The core team also provided wound care (cannulation sites, chest tubes, sternal incision) as well as daily follow up. $24 \mathrm{hr}$ "on call" support was provided those involved in care. After initial training of the experts, all efforts were directed towards development of a larger core team that could act as a resource for health care professionals. These experts successfully maintained continuity of care. An initial comprehensive infrastructure of supports was put into place by the core group to support primary caregivers of VAD patients and families. The comprehensive infrastructure included perfusionists (pre-implant equipment organization and perfusion), biomedical engineers (maintenance/service/education of the IKUS driving unit), transplant/heart failure services (pre-transplant patient management/listing), physiotherapy (rehabilitation after implantation), social work (family and patient support throughout the trajectory of illness), pharmacy (drug therapy), psychiatry (support of patients and families), haematology (anticoagulation), and trained nurses from critical care and the cardiology ward. Results: From October 2004 to November 2007, 10 Berlin Heart EXCOR. Pediatric Ventricular Assist Devices were implanted in pediatric patients with a diagnosis of cardiomyopathy or heart failure. Out of 9 patients, eight (88\%) were successfully transplanted, and $77 \%$ survived to hospital discharge. One patient was transitioned from the cardiac critical care unit to the cardiac ward on the Berlin Heart. One patient is currently being supported on the Berlin Heart. Conclusions: The complex care of a patient on a VAD needs a specialized team approach to cover all patient care needs. Ongoing interprofessional education continues to improve competency of care. Continuity of care was assured on all levels of service to guarantee the best possible outcomes.

Correspondence to Sarah Furness: sarah.furness@sickkids.ca

\section{BNP and heart failure in patients with a ventricular septal defect}

Michael A. Paul, Carl L. Backer, Helen J. Binns, Constantine Mavroudis, Catherine L. Webb, Ram Yogev, Wayne H. Franklin

\section{Children's Memorial Hospital, Chicago, IL, USA}

Background: Patients with a ventricular septal defect (VSD) are at risk for heart failure. Patients with heart failure due to a VSD are initially treated medically but often require surgical closure of the 
VSD. The specific medical treatment and surgical timing are often dictated by the degree of heart failure. The diagnosis of heart failure is based on signs and symptoms that are neither sensitive nor specific. In adults without congenital heart disease, b-type natriuretic peptide (BNP) has been shown to be a very sensitive and specific marker of heart failure. The utility of BNP as a marker of clinical heart failure in children with a VSD has yet to be determined. This study was designed to determine whether BNP is related to the degree of heart failure in patients with a VSD. Methods: A prospective, observational study was designed to evaluate BNP levels and other measures of heart failure. Eligible patients were $<2$ years old and scheduled to undergo surgical repair of a VSD. Those with significant heart disease other than VSD, atrial septal defect (ASD), patent foramen ovale (PFO), and patent ductus arteriosus (PDA) were excluded. Data were collected within two weeks immediately prior to surgery and included: echocardiographic measurements (left ventricular (LV) size, mass, and shortening fraction), electrocardiographic (ECG) findings (ventricular hypertrophy), Ross score, and BNP measurements. Weight gain prior to repair was determined by computing the weight z-score change/month. Twenty-four children met eligibility criteria and 19 patients were enrolled. BNP measures were log transformed and correlation with other measures of heart failure evaluated. A single subject with an outlier value of BNP $(937 \mathrm{pg} / \mathrm{ml})$ was excluded from correlation analysis. The study was approved by the hospital institutional review board. Results: Subjects were 58\% male with median age 6.0 months (1.3-13.6); 32\% had aneuploidy. Additional cardiac diagnoses included: ASD (16\%) and PFO (5\%). Measurements immediately prior to VSD repair were: BNP (geometric mean 38, median 32, IQR 19-67 pg/ml), LV diastolic dimension z-score (mean $0.9 \pm 1.8$ ), LV mass (mean $78 \pm 27 \mathrm{~g} / \mathrm{m}^{2}$ ), Ross score (mean $1.5 \pm 1.6$ ), weight gain (weight $z$-score change/ month, mean $-0.15 \pm 0.21$ ). Subject ECGs demonstrated $\mathrm{LVH}$ in $6 \%, \mathrm{RVH}$ in $22 \%$, and biventricular hypertrophy in $61 \%$ of subjects. There was a trend toward inverse correlation $(\mathrm{r}=-0.46$, $\mathrm{p}=0.07)$ between $\log (\mathrm{BNP})$ and weight gain. Conclusions: BNP in children with a VSD requiring surgical repair demonstrates a trend toward inverse correlation with weight gain. Several other measures of heart failure did not demonstrate correlation with BNP. The use of BNP as a marker of heart failure in children with a VSD merits further investigation.

Correspondence to Michael Paul: mpaul@childrensmemorial.org
An echocardiography simulator for congenital heart disease: concept and prototype design

Michael Weidenbach, Vit Razek, Florentine Wild, Jan Janousek

Department of Pediatric Cardiology, Heart Centre Leipzig, Leipzig, Germany

Background: Echocardiography is the most important diagnostic imaging in congenital heart disease (CHD). CHD are challenging due to the huge number of different lesions, the rarity of each and the emergency situation the patients often present. Becoming acquainted with the full range of $\mathrm{CHD}$ often needs years of training. We have therefore incorporated the most prevalent CHD lesions for training purposes in a simulator for echocardiography. Methods: The simulator is based on our previously described and evaluated simulator for two-dimensional (2D) echocardiography. 2D views are calculated form 3D data sets. 3D full volume data sets were recorded over 4 heart cycles using a real-time $3 \mathrm{MHz}$ transthoracic ultrasound probe (GE, Vivid 7). Data sets were stored and converted from proprietary format. These data sets are registered spatially and temporally with a virtual non-pathologic heart model. In addition to registration this heart model is used as guidance for the inexperienced echocardiographer to have an orientation about probe position in relation to the heart. After registration the 3D data can be scanned by a dummy probe attached to an electromagnetic position sensor placed on a dummy torso as if a real patient was examined. Results: We have been able to acquire $3 \mathrm{D}$ data sets of the most relevant lesions like VSD, ASD, TOF, TGA, ccTGA, AVSD, TAC, TGA/Senning etc. Acquisition was predominantly subcostal in neonates and apical in older patients. All data sets could be registered, achieving correct position of the data within the dummy torso. Anatomical correlation of the data sets with the heart model was not correct in most cases, due to the nature of the lesion. However, this was not judged as a predominant problem by most external reviewers, since the guidance by the heart model is most useful for the absolute beginners in echocardiography. These should start with anatomically normal hearts and proceed to CHD in a later stage of their training. Conclusions: Simulation of echocardiography using Real-time 3D echocardiographic data sets with our simulator is possible. There are, however, some limitations. 1) The current simulator consists of an adult dummy torso. A baby dummy is under construction. 
2) Currently there is no 3D Color Doppler in Full volume mode on commercial available scanners. We hope that further progress in scanner technology will enable us to incorporate Color Doppler information. 3) Resolution is not optimal using a $3 \mathrm{MHz}$ probe for neonates. 4) Incorporating virtual heart models of the appropriate lesion might be helpful for beginners. If the additional costs would be justified will be tested in the future. 5) Formal evaluation of the simulator for CHD has not been done yet but is on the agenda for the future.

Correspondence to Michael Weidenbach: michael.weidenbach@ medizin.uni-leipzig.de

\section{Ritual or evidence - a time for change?}

Deborah Lloyd Torowicz

\section{Children's Hospital of Philadelphia, Philadelpbia,} PA, USA

Background: Little empirical evidence exists to support the current standard of care in the postcatheterization recovery period in infants undergoing cardiac catheterization. Several studies with adult patients report wide variations in practice regarding time in bed as well as positioning. Foundations of care for adults, e.g. duration of bedrest time (6-24 hours) and positioning components (legs straight and lying flat and supine) have been refuted in the literature. Recent evidence supports that bed-rest time as well as position can be safely reduced yet are not implemented into practice. Hence, the standard of care in infants during the post-catheterization recovery period is based solely on past practice patterns in the adult patient. Methods: A comprehensive review of the literature using Medline, CINAHL, OVID and PubMed was conducted using the key terms: cardiac catheterization, complications, comfort, cost effectiveness, back pain, early ambulation, bed-rest time, positioning, and safety. Data collection tools adapted from Titler (2001) were used to summarize findings and grade the evidence. Studies reporting catheter sizes comparable to catheters used in pediatrics, 8 or 7 French (Bashore, Bates, Berger, et al., 2001), were considered for review and eliminated were studies that reported use of a compression device. Results: The duration of bed-rest time in adults in different treatment centers was successfully reduced from 12 to 6 hours, 6 to 4 hours, and from 4 to 2 hours with improved patient comfort, less pain, and no significant difference in bleeding, hematoma formation or pseudoaneurysms. The few studies that evaluated position change examined back rest elevation ranging from 150 to 600 at different periods during the postcatheterization recovery period and found comparable findings in regards to pain, comfort and vascular complications. Conclusions: The standard of practice during the post-catheterization recovery period is based on the current standard of care in adults, institutional or physician preference. Establishing evidence in the care of pediatric and neonatal patients is essential and may possibly allow infants to be held and comforted sooner by their caregiver and may reduce poor outcomes related to immobility, pain and agitation.

Correspondence to Deborah Lloyd Torowicz: torowicz@email. chop.edu 